\title{
Structural revisions of natural products by Computer Assisted Structure Elucidation (CASE) Systems
}

\author{
Mikhail Elyashberg ${ }^{1}$, Antony J. Williams ${ }^{2}$, Kirill Blinov ${ }^{1}$. \\ ${ }^{1}$ Advanced Chemistry Development, Moscow Department, 6 Akademik Bakulev Street, Moscow \\ 117513, Russian Federation. \\ ${ }^{2}$ Royal Society of Chemistry, US Office, 904 Tamaras Circle, Wake Forest, NC-27587

\section{Introduction}

2. An axiomatic approach to the methodology of molecular structure elucidation

3. The expert system Structure Elucidator: a short overview.

4. Examples of structure revision using an expert system

4.1 Revision of structures by reinterpretation of experimental data

4.2 Revision of structures by the application of chemical synthesis

4.3 Revision of structures by the reexamination of 2D NMR data

4.4 Structure selection on the basis of spectrum prediction

5. Conclusions

\section{Introduction}

Computer-Aided Structure Elucidation (CASE) is a scientific area of investigation initiated over forty years ago and on the frontier between organic chemistry, molecular spectroscopy and computer science. As a result of the efforts of many researchers, a series of so-called expert systems (ES) intended for the purpose of molecular structure elucidation from spectral data have been developed. Before the start of the 21 st century these systems were used primarily for the elaboration and examination of the CASE methodology. The systems created in this time period could be considered as research prototypes of analytical tools rather than production tools. In first decade of this century a radical change occurred in terms of the capabilities of these expert systems to elucidate the structures of new and complex (>100 heavy atoms) organic molecules from a collection of mass spectrometric and NMR data. Expert systems are now being used for the identification of natural products, as well as for the structure determination of their degradants and analysis of chemical reaction products. Examples of the application of ES systems for such purposes have been published elsewhere (see for instance ${ }^{1-9}$ ). Reviews of the state of the science in 
regards to CASE developments were produced by Jaspars ${ }^{10}$ (1999) and Steinbeck ${ }^{11}$ (2004). A comprehensive review of the current state of computer-aided structure elucidation and verification was recently published by this laboratory ${ }^{12}$. Other expert systems based on the analysis of 2D NMR spectra ${ }^{13-19}$ were discussed in that review article.

This article was initiated by the review of Nicolaou and Snider ${ }^{20}$ entitled "Chasing molecules that were never there: misassigned natural products and the role of chemical synthesis in modern structure elucidation" published in 2005. The review posits that both imaginative detective work and chemical synthesis still have important roles to play in the process of solving nature's most intriguing molecular puzzles. Another review entitled "Structural revisions of natural products by total synthesis" was recently presented by Maier $^{21}$. This work encompasses the time period between 2005 and 2009.

According to Nicolaou and Snider $^{20}$ around 1000 articles were published between 1990 and 2004 where the originally determined structures needed to be revised. Figuratively speaking, it means that 40-45 issues of the imaginary "Journal of Erroneous Chemistry" were published where all articles contained only incorrectly elucidated structures and, consequently, at least the same number of articles were necessary to describe the revision of these structures. The associated labor costs necessary to correct structural misassignments and subsequent reassignments are very significant and, generally, are much higher than those associated with obtaining the initial solution. From these data it is evident that the number of publications in which the structures of new natural products are incorrectly determined is quite large and reducing this stream of errors is clearly a valid challenge. The authors of the review ${ }^{20}$ comment that "there is a long way to go before natural product characterization can be considered a process devoid of adventure, discovery, and, yes, even unavoidable pitfalls". The review of Maier ${ }^{21}$ confirms this conclusion.

We believe that the application of modern CASE systems can frequently help the chemist to avoid pitfalls or, in those cases when the researcher is challenged, then the expert system can at least provide a cautionary warning. Our belief is based on the fact that molecular structure elucidation can be formally described as deducing all logical corollaries from a system of statements which ultimately form a partial axiomatic theory. These corollaries are all conceivable structures that meet the initial set of axioms ${ }^{22-24}$. The great potentiality of ES is due to the fact that these systems can be considered as an inference engine applicable to the knowledge presented the set of axioms. Particularly, the expert system Structure Elucidator (StrucEluc) ${ }^{12,25-29}$ developed by our group is based on the presentation of all initial knowledge in the form of a partial axiomatic theory. The system is 
capable of inferring all plausible structures from 1D and 2D NMR data even in those cases when the spectrum-structural information is very fuzzy (see below).

This system was used in our investigation for the following reasons. As discussed in a previous review article ${ }^{12}$ all available expert systems to perform structure elucidation using MS and 2D NMR data were reviewed. StrucEluc was demonstrated to be the most advanced system containing all intrinsic features contained within other systems but also has a series of additional features which make it capable of solving very complex real problems. Despite the fact that StrucEluc is a commercially available CASE program ongoing research continues to improve the performance of the platform. The system is installed in many structure elucidation laboratories around the world and has proven itself on many hundreds of both proprietary and non-proprietary structural problems. In his 2004 review ${ }^{11}$ Steinbeck notes that "the most promising achievements in terms of practical applicability of CASE system have been made using ACD/Labs' Structure Elucidator program... which combines both flexible algorithms for ab initio CASE as well as a large database for a fast dereplication procedure". The system has been markedly improved for the last 6 years since the cited review ${ }^{11}$ was published. It should be noted that during the same period of time only one new expert system has been described in the literature ${ }^{30}$. The system is intended to perform structure elucidation using ${ }^{1} \mathrm{H}$ and ${ }^{1} \mathrm{H}-{ }^{1} \mathrm{H}$ COSY spectra. Since the amount of structural information extracted from spectral data without the application of direct and long-range heteronuclear correlation experiments is limited, the system is applicable only to the identification of simple and modest sized molecules.

Nicolaou et $a l^{20}$ noted that the development of spectroscopic methods in the second half of the 20th century resulted in a revolution in the methodology of structure elucidation. We believe that the continued development of algorithms and accompanying software platforms and expert systems will further revolutionize structure elucidation. We are sure that the employment of expert systems will lead to significant acceleration in the progress of organic chemistry and natural products specifically as a result of reduced errors and increased efficiencies.

This review considers the application of CASE systems to a series of examples in which the original structures were later revised. We demonstrate how the chemical structure could be correctly elucidated if 2D NMR data were available and the expert system Structure Elucidator was employed. We will also demonstrate that if only 1D NMR spectra from the published articles were used then simply the empirical calculation of ${ }^{13} \mathrm{C}$ chemical shifts for the hypothetical structures frequently enables a researcher to realize that the structural hypothesis is likely incorrect. We also analyze a number of erroneous 
structural suggestions made by highly qualified and skilled chemists. The investigation of these mistakes is very instructive and has facilitated a deeper understanding of the complicated logical-combinatorial process for deducing chemical structures.

The multiple examples of the application of Structure Elucidator for resolving misassigned structures has shown that the program can serve as a flexible scientific tool which assists chemists in avoiding pitfalls and obtaining the correct solution to a structural problem in an efficient manner. Chemical synthesis clearly still plays an important role in molecular structure elucidation. The multi-step process requires the structure elucidation of all intermediate structures at each step, for which spectroscopic methods are commonly used. Consequently, the application of a CASE system would be very helpful even in those cases when chemical synthesis is the crucial evidence to identify the correct structure. We also believe that the utilization of CASE systems will frequently reduce the number of compounds requiring synthesis.

\section{An axiomatic approach to the methodology of molecular structure elucidation}

The history of development of CASE systems to date has convincingly demonstrated the point of view suggested 40 years $\mathrm{ago}^{22,23}$ that the process of molecular structure elucidation is reduced to the logical inference of the most probable structural hypothesis from a set of statements reflecting the interrelation between a spectrum and a structure. This methodology was implicitly used for a long time before computer methods appeared. Independent of computer-based methods the path to a target structure is the same and CASE expert systems mimic the approaches of a human expert. The main advantages of CASE systems are as follows: 1) all statements regarding the interrelation between spectra and a structure ("axioms") are expressed explicitly; 2) all logical consequences (structures) following from the system of "axioms" are completely deduced without any exclusions; 3 ) the process of computer-based structure elucidation is very fast and provides a tremendous saving in both time and labor for the scientist; 4) if the chemist has several alternative sets of axioms related to a given structural problem then an expert system allows for the rapid generation of all structures from each of the sets and identification of the most probable structure by comparison of the solutions obtained.

We describe below the main kinds of statements used during the process of structure elucidation. These can be conventionally divided in the following categories:

\section{Axioms and hypotheses based on characteristic spectral features.}


In accordance with the definition we refer to "axioms" as those statements that can be considered true based on prior experience. To elucidate the structure of a new unknown compound, the chemist usually uses spectrum-structure correlations established as a result of the efforts of several generations of spectroscopists. Statements reflecting the existence of characteristic spectral features plays a role in the basic axioms of structure elucidation theory. The general form of typical axioms belonging to this category can be presented as follows:

If a molecule contains a fragment $A_{i}$ then the characteristic features of fragment $A_{i}$ are observed in certain spectrum ranges $\left[X_{1}\right],\left[X_{2}\right], \ldots\left[X_{m}\right]$ which are characteristic for this fragment.

For example, if a molecule contains a $\mathrm{CH}_{2}$ group then a vibrational band around 1450 $\mathrm{cm}^{-1}$ is observed in the IR spectrum. If a molecule contains a $\mathrm{CH}_{3}$ group then two bands around 1450 and $1380 \mathrm{~cm}^{-1}$ appear. These axioms can be presented formally in the following way using the symbols of implication $(\rightarrow)$ and conjunction $(\Lambda)$ conventional in symbolic logic:

$$
\mathrm{CH}_{2} \rightarrow\left[1450 \mathrm{~cm}^{-1}\right] ; \mathrm{CH}_{3} \rightarrow[1380] \wedge\left[1450 \mathrm{~cm}^{-1}\right]
$$

Analogously, for characteristic ${ }^{13} \mathrm{C}$ NMR chemical shifts the following implications are also exemplar axioms:

$$
(\mathrm{C})_{2} \mathrm{C}=\mathrm{O} \rightarrow[200 \mathrm{ppm}], \quad(\mathrm{C})_{2} \mathrm{C}=\mathrm{S} \rightarrow[200 \mathrm{ppm}] .
$$

When characteristic spectral features are used for the detection of fragments that can be present in a molecule under investigation then the chemist usually forms statements for which a typical "template" is as follows:

If a spectral feature is observed in a spectrum range $\left[X_{j}\right]$ then the molecule contains at least one fragment of the set $A_{i}\left(X_{j}\right), A_{k}\left(X_{j}\right), \ldots A_{l}\left(X_{j}\right)$, where $A i, A k, \ldots A l$ are fragments for which the spectral feature observed in the range $\left[X_{j}\right]$ is characteristic, and the fragments form a finite set.

This statement is a hypothesis, not an axiom, because: $\mathrm{i})$ the feature $\mathrm{X}_{j}$ can be produced by some fragment which is not known as yet, ii) the feature $\mathrm{X}_{j}$ can appear due to some intramolecular interaction of known fragments. Therefore, if an absorption band is observed at $1450 \mathrm{~cm}^{-1}$ in an IR spectrum then the molecule can contain either $\mathrm{CH}_{2}$ or $\mathrm{CH}_{3}$ groups, both of them (band overlap at $1450 \mathrm{~cm}^{-1}$ is allowed), or the $1450 \mathrm{~cm}^{-1}$ band can be present as a result of the presence of another unrelated functional group. This statement can be expressed formally using the symbol for logical disjunction $(\mathrm{V}): 1450 \mathrm{~cm}^{-1} \rightarrow \mathrm{CH}_{2} \mathrm{~V} \mathrm{CH}$ $\mathrm{V} \alpha$, where $\alpha$ is a "sham fragment" denoting an unknown cause of the feature origin. For 
our ${ }^{13} \mathrm{C}$ NMR examples, we may obviously formulate the following hypothesis: $200 \mathrm{ppm} \rightarrow$ $(\mathrm{C})_{2} \mathrm{C}=\mathrm{O} \vee(\mathrm{C})_{2} \mathrm{C}=\mathrm{S}$. It is very important to have in mind that if $\mathrm{A}_{i} \rightarrow \mathrm{X}_{j}$ is true, then the inverse implication $\mathrm{X}_{j} \rightarrow \mathrm{A}_{i}$ can be true or not true. In other words, the presence of a characteristic spectral feature in a spectrum does not imply the presence of a corresponding fragment. A true implication is $\overline{X_{j}} \rightarrow \overline{A_{i}}$. This implication means that if the characteristic spectral feature $\mathrm{X}_{j}$ does not occur in a spectrum, then the corresponding fragment $\mathrm{A}_{i}$ is absent from the molecule under investigation. The latter statement can be considered as another equivalent formulation of the basic axiom.

All fragment combinations which may exist in the molecule can be logically deduced from the set of axioms and hypotheses by solving a logical equation ${ }^{22,23,31}$

$$
A\left(\mathrm{~A}_{i}, \mathrm{X}_{j}\right) \rightarrow\left\{S p\left(\mathrm{X}_{j}\right) \rightarrow C\left(\mathrm{~A}_{i}\right)\right\}
$$

Here $A\left(\mathrm{~A}_{i}, \mathrm{X}_{j}\right)$ is a full set of axioms and hypotheses reflecting the interrelation between fragments $\mathrm{A}_{i}$ and their spectral features $\mathrm{X}_{j}$ in all available spectra, $\operatorname{Sp}\left(\mathrm{X}_{j}\right)$ is the combination of spectral features observed in the experimental spectra and $C\left(\mathrm{~A}_{i}\right)$ is a logical function enumerating all possible combinations of the fragments $\mathrm{A}_{i}$ which may exist in a molecule. This equation has the following intuitively clear interpretation: if the axioms and hypotheses $A\left(\mathrm{~A}_{i}, \mathrm{X}_{j}\right)$ are true then the combinations of fragments described by the $C\left(\mathrm{~A}_{i}\right)$ function follow from the combination of spectral features $\operatorname{Sp}\left(\mathrm{X}_{j}\right)$ observed in the spectra. These considerations are evident when IR and 1D NMR spectra are used, but they are generally applicable to 2D NMR spectra also.

\section{Axioms and hypotheses of 2D NMR Spectroscopy.}

2D NMR spectroscopy is a method which, in principle, is capable of inferring a molecular structure from the available spectral data $a b$ initio without using any spectrumstructure correlations and additional suppositions. In some cases the 2D NMR data provides sufficient structural information to suggest a manageable set of plausible structures. This is a fairly common situation for small molecule with a lot of protons contained within the molecule. In practice the structure elucidation of large molecules by the ab initio application of 2D NMR data only (without 1D NMR spectrum-structure correlations) is generally impossible. The 1D and 2D NMR data are usually combined synergistically to obtain solutions to real analytical problems in the study of natural products.

Experience has shown ${ }^{25-29}$ that the size of a molecule is not a crucial obstacle for a CASE system based on 2D NMR data. The number of hydrogen atoms responsible for the propagation of structural information across the molecular skeleton and the number of 
skeletal heteroatoms are the most influential factors. An abundance of hydrogen atoms and a small number of heteroatoms generally eases the structure elucidation process rather markedly. To date we have failed to determine any specific dependence between molecular composition and the number of plausible structures deduced by an expert system because the different modes for solving a problem are chosen according to the nature of the specific problem (see Section 3). Moreover, the complexity of the problem is associated with many factors which cannot be identified before attempts are made to solve the problem. For instance, the complexity of the problem depends on whether the heavy atoms and their attached hydrogen atoms are distributed "evenly" around the molecular skeleton. If at least one "silent" fragment (i.e. having no attached hydrogens) is present in a molecule then it can interrupt a chain of HMBC and COSY correlations. As a result the number of structural hypotheses will increase dramatically as reported, for example, in the cryptolepine family ${ }^{28}$.

When 2D NMR data are used to elucidate a molecular structure then the chemist or an expert system mimics the manner of deducing conceivable structures from the molecular formula and a set of hypotheses matching the data from two-dimensional NMR spectroscopy. When we deal with a new natural product we must interpret a new 2D NMR spectrum or spectra. In this case we have no possibility to rely on "axioms" valid for the given spectrum-structure matrix so hypotheses which are considered as the most plausible are formed. These hypotheses are based on the general regularities which are the significant axioms of $2 D$ NMR spectroscopy. We will attempt to express these axioms in an explicit form and classify them.

There are of course various forms of 2D NMR spectroscopy, the most important and common of these being homonuclear ${ }^{1} \mathrm{H}-{ }^{1} \mathrm{H}$ and heteronuclear ${ }^{1} \mathrm{H}_{-}{ }^{13} \mathrm{C}$ spectroscopy. Even though heteronuclear interactions of the nature X1-X2 (X1 and X2 are magnetically active nuclei but not ${ }^{1} \mathrm{H}$ nor ${ }^{13} \mathrm{C}$ ) are possible such spectra are rare and, except for labeled materials, very difficult to acquire in general.

A necessary condition for the application of $2 \mathrm{D}$ data to computer assisted structure elucidation is the chemical shift assignment of all proton-bearing carbon nuclei, (i.e. all $\mathrm{CH}_{\mathrm{n}}$ groups where $\mathrm{n}=1-3$ ). This information is extracted from the HSQC (alternatively HMQC) data using the following axiom:

- If a peak $(\delta \mathrm{C}-i, \delta \mathrm{H}-i)$ is observed in the spectrum then the hydrogen atom $\mathrm{H}-i$ with chemical shift $\delta \mathrm{H}-i$ is attached to the carbon atom $\mathrm{C}-i$ having chemical shift $\delta \mathrm{C}-i$. 
The main sources of structural information are COSY and HMBC correlations which allow the elucidation of the backbone of a molecule. We refer to "standard" correlations ${ }^{32}$ as those that satisfy the following axioms reflecting the experience of NMR spectroscopists:

- If a peak $(\mathrm{H}-i, \mathrm{H}-k)$ is observed in a COSY spectrum, then a molecule contains the chemical bond $(\mathrm{C}-i)-(\mathrm{C}-k)$.

- If a peak $(\delta \mathrm{H}-i, \delta \mathrm{C}-k)$ is observed in a HMBC spectrum, then atoms $\mathrm{C}-i$ and $\mathrm{C}-k$ are separated in the structure by one or two chemical bonds:

$(\mathrm{C}-i)-(\mathrm{C}-k)$ or $(\mathrm{C}-i)-(\mathrm{X})-(\mathrm{C}-k), \mathrm{X}=\mathrm{C}, \mathrm{O}, \mathrm{N} \ldots$

By analogy, the main axiom associated with employing the NOE effect for the purpose of structure elucidation can be formulated in the following manner:

- If a peak ( $\delta \mathrm{H}-i, \delta \mathrm{H}-k)$ is observed in a NOESY (ROESY) spectrum, then the distance between the atoms $\mathrm{H}-i$ and $\mathrm{H}-k$ through space is less than $5 \AA$.

It is important to note that there is a distinct difference between the logical interpretations of the $1 \mathrm{D}$ and 2D NMR axioms. For example, for COSY there is a second equivalent form of the main axiom which can be declared as:

- If a molecule does not contain the chemical bond $(C-i)-(C-k)$, then no peak $(H-i, H-$ k) will observed in a COSY spectrum.

In this case the interpretation allows us to conclude that the absence of a peak $(\mathrm{H}-\mathrm{i}$, $H-k$ ) says nothing about the existence of a chemical bond $(C-i)-(C-k)$ in the molecule: i.e. the bond may exist or may not exist. Consequently, the expert system does not use the absence of 2D NMR peaks $(H-i, H-k)$ to reject structures containing the bond $(C-i)-(C-k)$. Analogous logic also applies to both HMBC and NOESY spectra.

While it is known that the listed axioms hold in the overwhelming majority of cases, there are many exceptions and these correlations are referred to as nonstandard correlations, $\mathrm{NSCs}^{32}$. Since standard and nonstandard correlations are not easily distinguished the existence of NSCs is the main hurdle to logically inferring the molecular structure from the 2D NMR data. If the 2D NMR data contain both undistinguishable standard and nonstandard correlations then the total set of "axioms" derived from the 2D NMR data will contain contradictions. This means that the correct structure cannot be inferred from these axioms and in this case the structural problem either has no solution or the solution will be incorrect: the set of suggested structures will not contain the genuine structure. Numerous examples of such situations will be considered in the next sections. 
Unfortunately as yet there are no routine NMR techniques which distinguish between 2D NMR signals belonging to standard and nonstandard correlations. In some fortunate cases the application of time consuming INADEQUATE and 1,1-ADEQUATE experiments, as well as $\mathrm{H} 2 \mathrm{BC}$ experiments is expected to help to resolve contradictions but these techniques are also based on their own axioms which can be violated.

\section{Structural hypotheses necessary for the assembly of structures.}

When chemical shifts in 1D and 2D NMR spectra are assigned and all 2D correlations are transformed into connectivities with other atoms in the skeletal framework then feasible molecular structures should be assembled from "strict fragments" (suggested on the basis of the 1D NMR, 2D COSY and IR spectra, as well as those postulated by the researcher) and "fuzzy fragments" determined from the 2D HMBC data. To assemble the structures it is necessary to make a series of responsible decisions, equivalent to constructing a set of axiomatic hypotheses. At least the following choices should be made:

- Allowable chemical composition(s): $\mathrm{CH}, \mathrm{CHO}, \mathrm{CHNO}, \mathrm{CHNOS}, \mathrm{CHNOCl}$, etc. The choice is made on the basis of chemical considerations and other additional information that may be available (sample origin, molecular ion cluster, etc.).

- Possible molecular formula (formulae) as selected from a set of possible accurate molecular masses. The suggestion of a molecular formula is crucial for CASE systems and is highly desirable in order to perform dereplication.

- Possible valences of each atom having variable valence: $\mathrm{N}(3$ or 5$), \mathrm{S}(2$ or 4 or 6$)$, $\mathrm{P}(3$ or 5$)$. If ${ }^{15} \mathrm{~N}$ and ${ }^{31} \mathrm{P}$ spectra are not available then, in principle, all admissible valences of these atoms should be tried. Obviously it is practically impossible to perform such a complete search. The application of a CASE system allows, in principle, the verification of all conceivable valence combinations and an example is reported in section 4.1 .

- Hybridization of each carbon atom: $s p ; s p^{2} ; s p^{3} ;$ not defined.

- Possible neighborhoods with heteroatoms for each carbon atom: $f b$ (forbidden), $o b$ (obligatory), not defined. An example of a typical challenge: does $\mathrm{C}(\delta=103 \mathrm{ppm})$ indicate a carbon in the $s p^{2}$ hybridization state or in the $s p^{3}$ hybridization state but connected with two oxygens by ordinary bonds?

- Number of hydrogen atoms attached to carbons that are the nearest neighbors to a given carbon (determined, if possible, from the signal multiplicity in the ${ }^{1} \mathrm{H}$ NMR spectrum). This decision may be rather risky and therefore such constraints should be used only with great caution and in those cases where no signal overlap occurs 
and signal multiplicity can be reliably determined as in the case of methyl group resonances that are typically singlets or doublets.

- Maximum allowed bond multiplicity: 1 or 2 or 3 . The main challenge relates to the triple bond. Strictly speaking it can be solved reliably only based on either IR or Raman spectra.

- List of fragments that can be assumed to be present in a molecule according to chemical considerations or based on a fragment search using the ${ }^{13} \mathrm{C} N M R$ spectrum to search the fragment DB. The chemical considerations usually arise from careful analysis of the NMR spectra related to known natural products that have the same origin and similar spectra. The presence of the most significant functional groups $(\mathrm{C}=\mathrm{O}, \mathrm{OH}, \mathrm{NH}, \mathrm{C} \equiv \mathrm{N}, \mathrm{C} \equiv \mathrm{C}, \mathrm{C} \equiv \mathrm{CH}$ etc.) can be suggested from both IR and Raman spectra when the corresponding assumptions are not contradicted by the NMR data and molecular formula of the unknown. Within an expert system such as Structure Elucidator a list of obligatory fragments can be automatically offered for consideration by the chemist with them making the final decision in regards to inclusion.

- List of fragments which are forbidden within the given structural problem. These include fragments unlikely in organic chemistry: for example, a triple bond in small cycles or an O-O-O connectivity, etc. Additionally substructures which are uncommon in the chemistry of natural products (for instance, a 4-membered cycle). IR and Raman spectra can also hint at the specification of forbidden fragments, and the axiom $\overline{X_{j}} \rightarrow \overline{A_{i}}$ is usually a rather reliable basis for making a particular decision. For example, if no characteristic absorption bands are observed in the region $3100-3700 \mathrm{~cm}^{-1}$, then an alcohol group will be absent from the unknown. This structural constraint which can be obtained very simply leads to the rejection of a huge number of conceivable structures containing the alcohol group (it is expected that the total number of isomers corresponding to a medium size molecule is comparable with the Avogadro constant).

It should be evident that at least one poor decision based on the points listed above would likely lead to a failure to elucidate the correct structure. We will see examples of this below.

If we generalize all axioms and hypotheses forming the partial axiomatic theory of a given molecule structure elucidation then we will arrive at the following properties which should be logically analyzed: 
- Information is fuzzy by nature, i.e. there are either 2 or 3 bonds between pairs of $\mathrm{H}-i$ and $\mathrm{C}-k$ atoms associated with a two-dimensional peak $(i, k)$ in the HMBC spectrum).

- Not all possible correlations are observed in the 2D NMR spectra, i.e., information is incomplete.

- The presence of nonstandard correlations (NSCs) frequently results in contradictory information.

- The number of NSCs and their lengths are unknown and signal overlap leads to the appearance of ambiguous correlations. Information is otherwise uncertain.

- Information can be false if a mistaken hypothesis is suggested.

- Information contained within the "structural axioms" reflects the opinion of the researcher and the information is, therefore, subjective, and typically based on biosynthetic arguments.

Taking into consideration the information properties above we can assume that the human expert is frequently unable to search all plausible structural hypotheses. Therefore, it is not surprising that different researchers arrive at different structures from the same experimental data and as a result, articles revising previously reported chemical structures are quite common as described in the introduction. Considering the potential errors that can combine in the decision making process associated with structure elucidation it is actually quite surprising that chemists are so capable of processing such intricate levels of spectrum-structure information and successfully extracting very complex structures at all. To assist the chemist to logically process the initial information a computer program that would be capable of systematically generating and verifying all possible structural hypotheses from ambiguous information would be of value. Structure Elucidator (StrucEluc $^{25-29}$ comprises a software program and series of algorithms which was specifically developed to process fuzzy, contradictory, incomplete, uncertain, subjective and even false spectrum-structural information. The program even provides suggestions regarding potential fallacies in the extracted information and warns the user. In the framework of the system each structural problem is automatically formulated as a partial axiomatic theory. Axioms and hypotheses included in the theory are analyzed and processed by sophisticated and fast algorithms which are capable of searching and verifying a huge number of structural hypotheses in a reasonable time. Fast and accurate NMR chemical shift prediction algorithms (see Section 3) are the basis for detection and rejection of incorrect structural conclusions following from poor initial input. 
As mentioned above, in this article the expert system Structure Elucidator developed by our group was used to demonstrate the potential of CASE systems as a tool for revealing incorrect structures and for their revision. More importantly we will show that the application of StrucEluc can be considered as an aid to avoid pitfalls and prevent the elucidation of incorrect structures. The many different features of this system have been discussed previously in a myriad of publications. However, to enable this article to be selfcontained and assist the reader in terms of understanding the main procedures of the platform we provide a short overview of StrucEluc.

\section{The expert system Structure Elucidator: a short overview.}

The expert system Structure Elucidator (StrucEluc) was developed towards the end of the 1990s. For the last decade it has been in a state of ongoing development and improvement of its capabilities. The areas of focused development were determined by solving many hundreds of problems based on the elucidation of structures of new natural products. The different strategies for solving the problems using StrucEluc, as well as the large number of examples to which we have applied the system are reported in manifold publications and were reviewed recently ${ }^{33}$. A very detailed description of the system can be found in a review ${ }^{12}$ and we will not repeat that analysis in this manuscript. Rather, in this section we will give a very short explanation of the algorithms underpinning the system as well as specify the various operation modes that provide a high level of flexibility to the software.

Generally, the purpose of the system is to establish topological and spatial structures, as well as the relative stereochemistry of new complex organic molecules from highresolution mass spectrometry (HRMS) and 2D NMR data. Mass spectra are used to determine the most appropriate molecular formula for an unknown. The availability of an extensive knowledgebase within StrucEluc allows the application of spectrum-structural information accumulated by several generations of chemists and spectroscopists to the task of computer-assisted structure elucidation. The knowledge can be divided into two segments: factual and axiomatic knowledge.

The factual knowledge consists of a database of structures $(420,000$ entries) and a fragment library $\left(1,700,000\right.$ entries) with the assigned ${ }^{1} \mathrm{H}$ and ${ }^{13} \mathrm{C}$ NMR spectra (subspectra). There is also a library containing 207,000 structures and their assigned ${ }^{13} \mathrm{C}$ and ${ }^{1} \mathrm{H}$ NMR spectra used for the prediction of ${ }^{13} \mathrm{C}$ and ${ }^{1} \mathrm{H}$ chemical shifts from input chemical structures. 
The axiomatic knowledge includes correlation tables for spectral structural filtering by ${ }^{13} \mathrm{C}$ and ${ }^{1} \mathrm{H}$ NMR spectra and an Atom Property Correlation Table (APCT). The APCT is used to automatically suggest atom properties as outlined in the previous section. A list of fragments that are unlikely for organic chemistry (BADLIST) can also be related to axiomatic knowledge of the system.

Firstly, peak picking is performed in the $1 \mathrm{D}{ }^{1} \mathrm{H},{ }^{13} \mathrm{C}$ and $2 \mathrm{D}$ NMR spectra. Spectral data for ${ }^{15} \mathrm{~N},{ }^{31} \mathrm{P}$ and ${ }^{19} \mathrm{~F}$ can be also used if available. For the 2D NMR spectra the coordinates of the two-dimensional peaks are automatically determined in the HSQC (HMQC), COSY and HMBC spectra and the corresponding pairs of chemical shifts are then fed into the program. As a result of the 2D NMR data analysis the program transforms the 2D correlations into connectivities between skeletal atoms and then a Molecular Connectivity Diagram (MCD) is created by the system. The MCD displays the atoms $\mathrm{XH}_{\mathrm{n}}(\mathrm{X}=\mathrm{C}, \mathrm{N}, \mathrm{O}$, etc.; $\mathrm{n}=0-3)$ together with the chemical shifts of the skeletal and attached hydrogen atoms. Each carbon atom is then automatically supplied with the properties of hybridization, different possible neighborhoods with various heteroatoms and so on for which the APCT is used. This procedure is performed with great caution, and a property is specified only in those cases when both the ${ }^{13} \mathrm{C}$ and ${ }^{1} \mathrm{H}$ chemical shifts support it. In all other cases the label not defined is given to the property. All properties can be inspected and revised by the researcher. Most frequently the goal of revising the atom properties is to reduce the uncertainty of the data to shorten the time associated with structure generation and to restrict the size of the output structural file. The user may also simply connect certain atoms shown on the MCD by chemical bonds to produce certain fragments and involve them in the elucidation process. Revision should be performed wisely so as to prevent incorrect outcomes. At the same time different variants of the atom property settings and the inclusion of fragments by adding new bond connectivities produces a set of different axioms that may be tested by subsequent structure generation. The MCD also displays all connectivities between the corresponding atoms (see Figure 24 as an example) and this allows the researcher to perform a preliminary evaluation of the complexity of the problem.

In accordance with 2D NMR axioms (Section 2) the default lengths of the COSYconnectivities are one bond $\left({ }^{3} J_{\mathrm{HH}}\right)$, while the lengths of the HMBC-connectivities vary from two to three bonds $\left({ }^{2-3} J_{\mathrm{CH}}\right)$. We refer to these connectivities as standard. The program starts with the logical analysis of the COSY and HMBC data to check them for the presence of connectivities with nonstandard lengths (corresponding to ${ }^{4-6} J_{\mathrm{HH}, \mathrm{XH}}$ correlations). The presence of nonstandard correlations (NSCs) can lead to the loss of the 
correct structure by the violation of the 2D NMR axioms and it is crucial to detect their presence or absence in order to solve the problem. When they are present it is important to estimate both the number and lengths of the nonstandard correlations. The algorithm performing the checking of the $2 \mathrm{D}$ NMR data ${ }^{32,34}$ is rather sophisticated and performs logical analysis of the 2D NMR data. The conclusion is based on the rule referred as ad absurdum. The algorithm is heuristic and we have found that it is capable of detecting NSCs in $\sim 90 \%$ of cases $^{27}$.

If logical analysis indicates that the data are free of nonstandard correlations then the next step is strict structure generation from the MCD. Two modes of strict structure generation are provided - the Common Mode and the Fragment Mode. The Common Mode is used if the molecular formula contains many hydrogen atoms which can be considered as the mediators of structural information and contribute to the possibility of extracting rich connectivity content from the 2D NMR data. The Common Mode implies structure generation from free atoms and fragments that were drawn by hand on the MCD (for instance, $\mathrm{O}-\mathrm{C}=\mathrm{O}, \mathrm{O}-\mathrm{H}$, etc.). If the double bond equivalent (DBE) value is small then the total number of connectivities is usually large and hence the number of restrictions is enough to complete structure generation in a short time. It is usually measured in seconds or minutes as can be seen in examples given in Section 4.

Our experience shows ${ }^{28}$ that such situations can occur when the number of constraints is not enough to obtain a structural file of a manageable size in an acceptable time. It means that the structural information contained within the 2D NMR data is not complete (see Section 2). This happens when the molecular formula contains only a few hydrogen atoms or when there is severe signal overlap in the NMR spectra and, as a result, too many ambiguous correlations. Alternatively the analyzed molecule may be too large or complex, for example, 100 or more skeletal atoms with many heteroatoms would be very challenging. In some cases all of these factors can occur simultaneously and the molecule under study may be large, devoid of hydrogen atoms and rich in the number of heteroatoms. In such situations the Fragment Mode has been shown to be very helpful, and for this purpose the Fragment Library is used. The program performs a fragment search in the library using the ${ }^{13} \mathrm{C}$ NMR spectrum as the basis of the search. All fragments whose sub-spectra fit with the experimental ${ }^{13} \mathrm{C}$ spectrum are selected. The program then analyses the set of Found Fragments, reveals the most appropriate ${ }^{28}$ and includes them in a series of molecular connectivity diagrams. Structure generation is then performed from the full set of MCDs and the generated structures are collected in a merged file. If no appropriate fragments were found in the Fragment Library then the researcher can create a User 
Fragment Library containing a set of fragments that belong to a specific class of organic molecules related to the unknown substance. The effectiveness of such an approach has previously been proven on a series of difficult problems ${ }^{7-9}$. If the researcher wants to include a set of specific User Fragments in the structure elucidation then the program can assign the experimental chemical shifts to carbon atoms within the fragments and include these fragments directly into the MCD.

If nonstandard connectivities are identified in the 2D NMR data then strict generation is not applicable as the 2D NMR data become contradictory. Unfortunately, the exact number of nonstandard connectivities and their lengths cannot be determined during the process of checking the MCD. Only a minimum number of NSCs can be found automatically. To perform structure generation from such uncertain and contradictory data, an algorithm referred to as Fuzzy Structure Generation (FSG) has been developed ${ }^{34}$. This mode allows structure generation even under those conditions when an unknown number of nonstandard connectivities with unknown lengths are present in the data. To remove the contradictions the lengths of the nonstandard correlations have to be augmented by a specific number of bonds depending on the kind of coupling $\left({ }^{4} J_{\mathrm{HH}, \mathrm{CH}},{ }^{5} \mathrm{~J}_{\mathrm{HH}, \mathrm{CH}}\right.$, etc.). The problem is formulated as follows: find a valid solution provided that the 2D NMR data involves an unknown number $m(m=1-15)$ of nonstandard connectivities and the length of each of them is also unknown.

Fuzzy structure generation is controlled by parameters that make up a set of options. The two main parameters are: $m$ - number of nonstandard connectivities and $a$ - the number of bonds by which some connectivity lengths should be augmented. Since 2D NMR spectral data cannot deliver definitive information regarding the values of these variables, both of them can be determined only during the process of fuzzy structure elucidation. We have concluded that in many cases the problem can be considerably simplified if the lengthening of the $m$ connectivities is replaced by their deletion (in this case the real connectivity length is not needed). When set in the options the program can ignore the connectivities by deleting connectivity responses that have to be augmented (the parameter $a=x$ is used in these cases). As in the process of FSG the program tries to perform structure generation from many submitted connectivity combinations. The total time consumed for this procedure is usually larger than in the case of strict structure generation for the same molecule if all connectivities had only standard lengths.

The efficiency of this approach was verified by the examination of more than 100 real problems with initial data containing up to 15 nonstandard connectivities differing in length from the standard correlations by 1-3 bonds. To the best of our knowledge StrucEluc 
is presently the only system that includes mathematical algorithms enabling the search for contradictions as well as their elimination and, therefore, is the only system that can work with many of the contradictions that exist in real 2D NMR data.

All structures that are generated in the modes discussed above are sifted through the spectral and structural filters in such a manner that the output structural file contains only those isomers which satisfy the spectral data, the system knowledge (factual and axiomatic) and the hypotheses of the researcher as true. The structures of the output file are supplied with both the ${ }^{13} \mathrm{C}$ and ${ }^{1} \mathrm{H}$ chemical shift assignments. The next step is the selection of the most probable structure from the output file. This procedure is performed using empirical ${ }^{13} \mathrm{C}$ and ${ }^{1} \mathrm{H}$ NMR chemical shift prediction previously described in detail ${ }^{12,35-37}$. Since an output file may be rather big (hundreds, thousands and even tens of thousands of structures) very fast algorithms for NMR spectrum prediction are necessary.

The following three-level hierarchy for chemical shift calculation methods has been implemented into StrucEluc:

- Chemical shift calculation based on additive rules (the incremental method). The program based on this algorithm ${ }^{37}$ is extremely fast. It provides a calculation speed of 6000-10,000 chemical shifts per second with the average deviation of the calculated chemical shifts from the experimental shifts equal to $d_{I}=1.6-1.8 \mathrm{ppm}$ (the symbol $I$ is used to designate the incremental method).

- Chemical shift calculation based on an artificial neural net $(\mathrm{NN})$ algorithm $^{35,} 37$. This algorithm is a little slower (4000-8000 chemical shifts per second) and its accuracy is slightly higher $-d_{N}=1.5-1.6 \mathrm{ppm}$. During the ${ }^{13} \mathrm{C}$ chemical shift prediction the algorithm takes into account the configuration of stereocenters in 5and 6-membered cycles.

- Chemical shift calculation based on HOSE-code ${ }^{38}$ (Hierarchical Organization of Spherical Environments). This approach is also referred to as the fragmental approach because the chemical shift of a given atom is predicted as a result of search for its "counterparts" having similar environment in one or more reference structures. The program also allows for stereochemistry, if known, of the reference structures. The spectrum predictor employs a database containing 207,000 structures with assigned ${ }^{13} \mathrm{C}$ and ${ }^{1} \mathrm{H}$ chemical shifts. For each atom within the molecule under investigation, related reference structures used for the prediction can be shown with their assigned chemical shifts. This allows the user to understand the origin of the predicted chemical shifts. This approach provides accuracy similar or commonly better than the neural nets approach. In this article the average 
deviation for $d_{H O S E}$ will be denoted as $d_{A}$. A shortcoming of the method is that it is not very fast with the prediction speed varying between several seconds to tens of seconds per structure depending on the size and complexity of a molecule.

To select the most probable structure the following three-step methodology is common within StrucEluc:

- ${ }^{13} \mathrm{C}$ chemical shift prediction for the output file is performed using an incremental approach. For a file containing tens of thousands of structural isomers the calculation time is generally less than several minutes. Next, redundant identical structures are removed. Since different deviations $d_{I}$ corresponds to duplicate structures with different signal assignments the structure with the minimum deviation is retained from each subset of identical structures (i.e., the "best representatives" are selected from each family of identical structures).

- ${ }^{13} \mathrm{C}$ chemical shift prediction for the reduced output file is performed using neural nets. Isomers are then ranked by ascending $d_{N}$ deviation and our experiences show that if the set of used axioms is true and consistent the correct structure is commonly in first place with the minimal deviation or is at least among the first several structures at the beginning of the list.

- ${ }^{13} \mathrm{C}$ chemical shift calculation for the first 20-50 structures from the ranked file is then performed using the fragmental (HOSE) method. Isomers are then ranked by ascending $d_{A}$ deviation to check if the structure distinguished by $\mathrm{NN}$ is preferable when both methods are used. Ranking by $d_{A}$ values is considered as more exacting and the value $d_{A}(1) \leq 1.5-2.5 \mathrm{ppm}$ is usually acceptable to characterize the correct structure.

If the difference between the deviations calculated for the first and second ranked structures is small $\left[d_{A}(2)-d_{A}(1)<0.2 \mathrm{ppm}\right]$ then the final determination of the preferable structure is performed by the expert. It was noticed ${ }^{27}$ that a difference value $d_{A}(2)-d_{A}(1)$ of $1 \mathrm{ppm}$ or more can be considered as a sign of high reliability of the preferable structure. Generally the choice is reduced to between two or, less frequently, three structures. In difficult cases, the ${ }^{1} \mathrm{H}$ NMR spectra can be calculated for a detailed comparison of the signal positions and multiplicities in the calculated and experimental spectra. Solutions that may be invalid are revealed by a large deviation of the calculated ${ }^{13} \mathrm{C}$ spectrum from the experimental spectrum for the first structure of the ranked file. For instance, if $d_{A}(1)>3-4$ ppm the solution should be checked using fuzzy structure generation. The reduced $d_{A}(1)$ 
value found as a result of fuzzy structure generation should be considered as hinting towards the presence of one or more nonstandard connectivities. A deviation of 3-4 ppm or more is usually considered as a warning that the initially preferred structure may be incorrect. The NOESY spectrum can also give valuable structural information (spatial constraints) at this step. The databases of structures and fragments included into system knowledgebase can be used for dereplication of the identified molecule and comparison of the NMR spectra with spectra of similar compounds.

As we have shown recently ${ }^{39}$ the HOSE-code based ${ }^{13} \mathrm{C}$ chemical shift prediction can be used as a filter for distinguishing one or more of the most probable stereoisomers of the elucidated structure. To determine the relative stereochemistry of this structure and to calculate its 3D model an enhancement to the program was introduced which can use 2D NOESY/ROESY spectra and a Genetic Algorithm ${ }^{40}$.

A general flow diagram for StrucEluc summarizing the main steps for analysis of data from an unknown sample to produce the structural formula of the molecule is shown in Figure 1. 


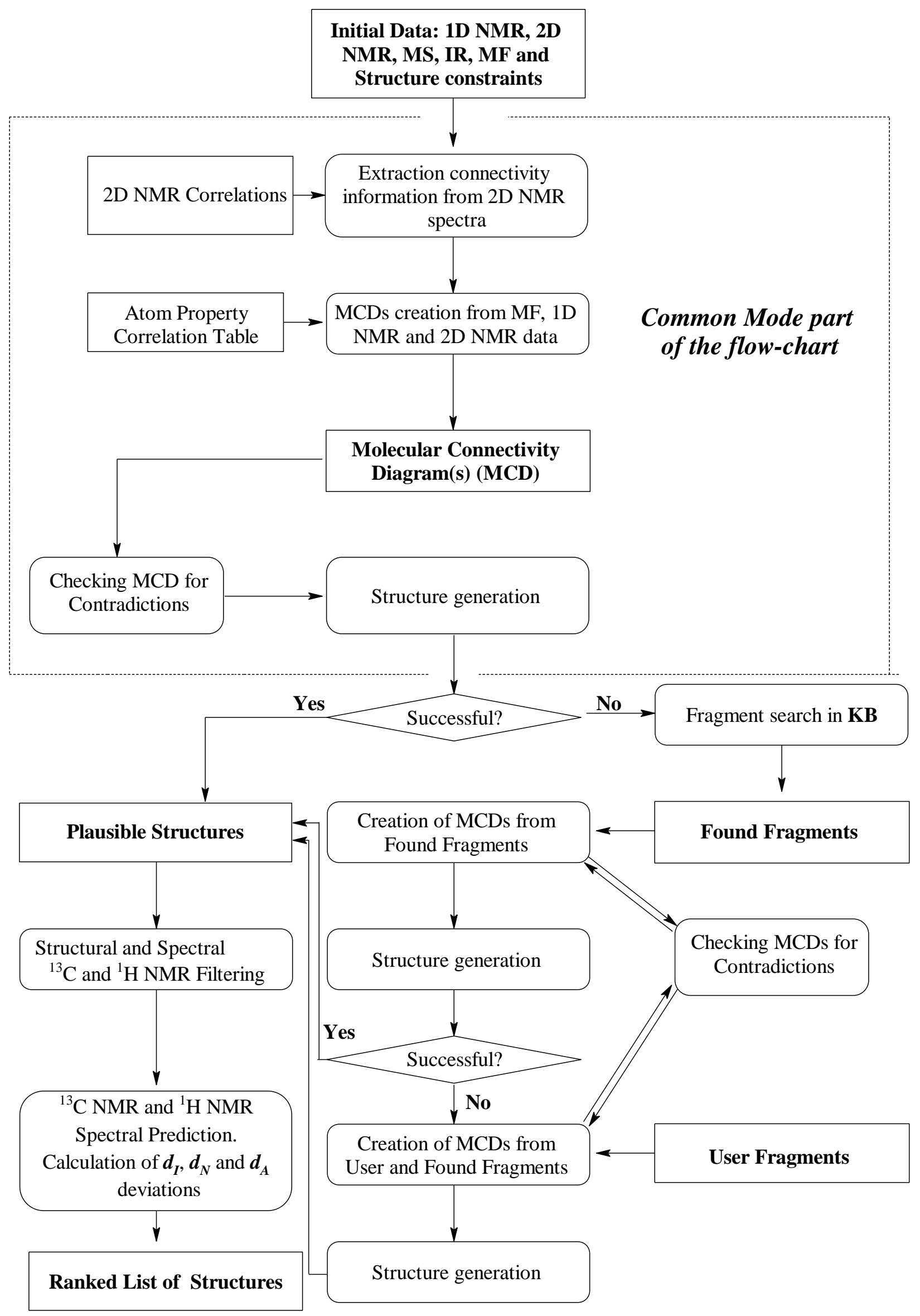

Figure 1. The flow diagram and decision tree for the application of StrucEluc. 


\section{Examples of structure revision using an expert system.}

In this section a series of articles are reviewed where an incorrect structure was initially inferred from the MS and NMR data and then later revised in later publications. In so doing we will demonstrate how the problem would have been solved if the StrucEluc system was used to process the initial information from the very beginning. The partial axiomatic theories were formed by the system from the spectrum-structure data and suggestions from the researchers presented in the corresponding articles.

The number of new natural products separated and published in the literature each year is huge. Obviously it is impossible for a scientific group to verify all structures presented in all articles. Therefore to choose the appropriate publications for consideration in this article we were forced to rely on those publications where the earlier identified structures were revised. Many references related to such structures were found in a review $^{20}$ covering the time period 1990-2005, while a series of later publications were revealed via an internet search. As a result we chose publications that were easily accessible. We then selected articles where the 2D NMR data were presented for the original structures (in the best cases - both for original and revised ones). With these data it was possible to analyze the full process of moving from the original spectra to the most probable structure and then clearly identify those points where questionable hypotheses led to the incorrect structures. If the 2D NMR data were not available within an article then it was only possible to assess the quality of the suggested structure on the basis of ${ }^{13} \mathrm{C} \mathrm{NMR}$ spectrum prediction.

It was difficult to decide how the various cases of structure revision could be classified. In the final analysis all problems were divided into four categories depending on the method or combination of methods which allowed us to reassign the original structure. We suggest that the following approaches can be distinguished: reinterpretation of experimental data, reexamination of the 2D NMR data, application of chemical synthesis, and ${ }^{13} \mathrm{C}$ NMR spectrum prediction. The reinterpretation of experimental data is required in those cases, for example, when an incorrect molecular formula is suggested, wrong fragments were suggested or artifacts in the 2D spectral data were taken as real signals, etc. In all cases it is impossible to obtain the correct structure. The reinterpretation of $2 \mathrm{D}$ data is necessary when a human expert misinterpreted the data because they were unable to enumerate all possible structures corresponding to the data.

\subsection{Revision of structures by reinterpretation of experimental data}


Randazzo et al ${ }^{41}$ isolated two new compounds, named halipeptins $\mathrm{A}$ and $\mathrm{B}$, from the marine sponge Haliclona sp. Their structures were determined by extensive use of $1 \mathrm{D}$ and 2D NMR (including ${ }^{1} \mathrm{H}-{ }^{15} \mathrm{~N}$ HMBC), MS, UV and IR spectroscopy assuming that these compounds belong to a class of materials with an elemental formula containing only CHNO, this assumption being an axiom. Halipeptin A showed an ion peak at $m / z 627.4073\left[(\mathrm{M}+\mathrm{H})^{+}\right]$in the high resolution fast atom bombardment mass spectrum (HRFABMS) consistent with a molecular formula of $\mathrm{C}_{31} \mathrm{H}_{54} \mathrm{~N}_{4} \mathrm{O}_{9}$ (calculated 627.3969 for $\mathrm{C}_{31} \mathrm{H}_{55} \mathrm{~N}_{4} \mathrm{O}_{9}$ with $\Delta \mathrm{m}=0.0104$, i.e. $16.6 \mathrm{ppm}$ ). The following structure (1) was suggested for halipeptin A (the suggested chemical shift assignment for the carbon and nitrogen nuclei is shown to simplify the observation of changes in the shift assignment when the structure is revised):

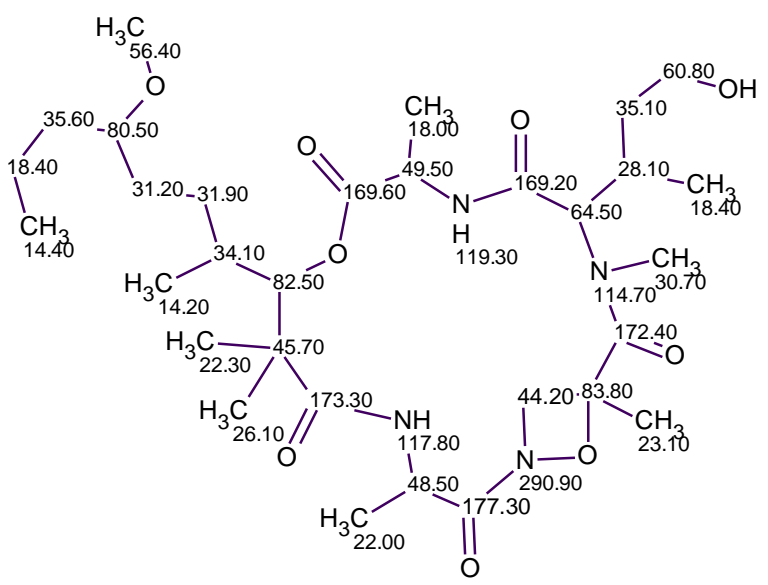

1

A four-membered ring cycle is known to occur very seldom in natural products. The authors $^{41}$ commented that a four-membered ring containing an $\mathrm{N}-\mathrm{O}$ bond appears to be a rather intriguing and unprecedented moiety. The presence of an $\mathrm{N}-\mathrm{O}$ bond was inferred from an IR band at $1446 \mathrm{~cm}^{-1}$ which was considered characteristic for an N-O bond as stretching in this range has already been observed in similar systems. Taking into account the axioms and accompanying examples described within the first group above such a consideration, in our opinion, is not convincing. The occurrence of this band does not contradict the presence of this specific fragment, but it also does not provide absolute evidence for the presence of the fragment in the analyzed structure. Moreover, all compounds containing $\mathrm{CH}_{2}$ groups also absorb in this region ${ }^{42}$. The unusual experimental chemical shift ( $\delta_{\mathrm{N}}=290.9 \mathrm{ppm}, \mathrm{NH}_{3}$ as reference) of the nitrogen nucleus associated with the hypothetical four-membered ring (the typical experimental $\delta_{\mathrm{N}}$ values in reference compounds used by Randazzo et al are 110-120 ppm) was explained in terms of the ring strain in the oxazetidine system. The large ${ }^{1} J_{\mathrm{CH}}$ values of 147.4 and $149.4 \mathrm{~Hz}$ observed for the two methylene protons, which is in excellent agreement with previously reported 
couplings for these ring systems, were considered as further support for the presence of this uncommon fragment.

To compare the suggested structure 1 with the results obtained from the StrucEluc software, the postulated molecular formula $\mathrm{C}_{31} \mathrm{H}_{55} \mathrm{~N}_{4} \mathrm{O}_{9}$ and spectral data including ${ }^{13} \mathrm{C}$ and ${ }^{15} \mathrm{~N}$ NMR spectra, HSQC, ${ }^{1} \mathrm{H}-{ }^{13} \mathrm{C}$ and ${ }^{1} \mathrm{H}-{ }^{15} \mathrm{~N}$ HMBC were used as input for the program. It was assumed that all axioms and hypotheses are consistent, that the valences of all nitrogen atoms are equal to 3 , and that $\mathrm{C} \equiv \mathrm{C}$ and $\mathrm{C} \equiv \mathrm{N}$ bonds were forbidden while the $\mathrm{N}-\mathrm{O}$ bond was permitted. No constraints on the ring cycle sizes were imposed. Molecular structure generation was run from the Molecular Connectivity Diagram (MCD) ${ }^{26}$ produced by the system and provided the result: $k=6 \rightarrow 4 \rightarrow 4, t_{g}=0.1 \mathrm{~s}$. This notation indicates that 6 structures were generated in $0.1 \mathrm{~s}$, and two sequenced operations - spectral-structural filtering and the removal of duplicates yielded four different structures. ${ }^{13} \mathrm{C}$ NMR spectrum prediction allowed us to select structure $\mathbf{2}$ as the most probable according to the minimal values of the mean average deviations $\left(d_{A} \cong d_{N}=3.6 \mathrm{ppm}\right)$ of the experimental ${ }^{13} \mathrm{C}$ chemical shifts from calculated ones. These different approaches of NMR prediction have been discussed in more detail elsewhere ${ }^{12,35}$ and shortly characterized in Section 3. They are included in the ACD/NMR Predictors software ${ }^{43}$ and implemented into StrucEluc.

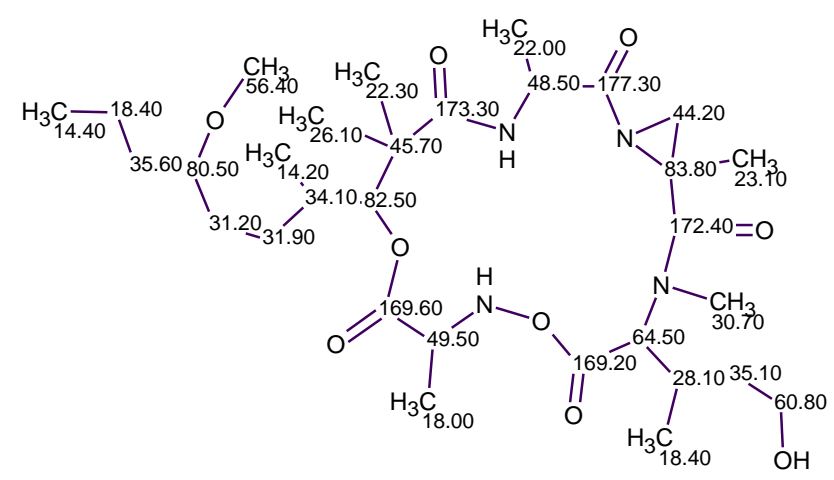

2

Structure $\mathbf{1}$ has not been generated. The deviations obtained are twice as large as the value of the calculation accuracy (1.6-1.8 ppm) but in cases such as this a decision regarding the structure quality is taken after analyzing the maximum deviations. A linear regression plot obtained using both HOSE and NN chemical shift predictions is presented in Figure 2. The graph and prediction limits were calculated using options available within the graphing program (Microsoft Excel). The graph shows that there is a single point lying outside the prediction limits and that the difference between the experimental (83.8) and calculated (45 ppm) chemical shifts is equal to about $40 \mathrm{ppm}$. This suggests that i) structure $\mathbf{2}$ is certainly wrong, ii) it is probable that at least one nonstandard correlation is present in the 2D NMR data. According to the general methodology inherent to the StrucEluc system, Fuzzy 
Structure Generation (FSG) ${ }^{34}$ should be used in such a situation. FSG was therefore executed and the presence of one NSC of an unknown length was assumed. The results are: $k=304 \rightarrow 284 \rightarrow 183$ and $t_{g}=35 \mathrm{~s}$. Figure 3 shows the first three structures of the output file ranked in order of increasing deviations following ${ }^{13} \mathrm{C}$ spectrum prediction. Structure $\mathbf{1}$ as suggested by the authors ${ }^{41}$ was ranked first, which means that they indeed inferred the best structure among all possible structures from the initial data (axioms). The crucial axiom influencing the final solution is the assumed molecular formula.

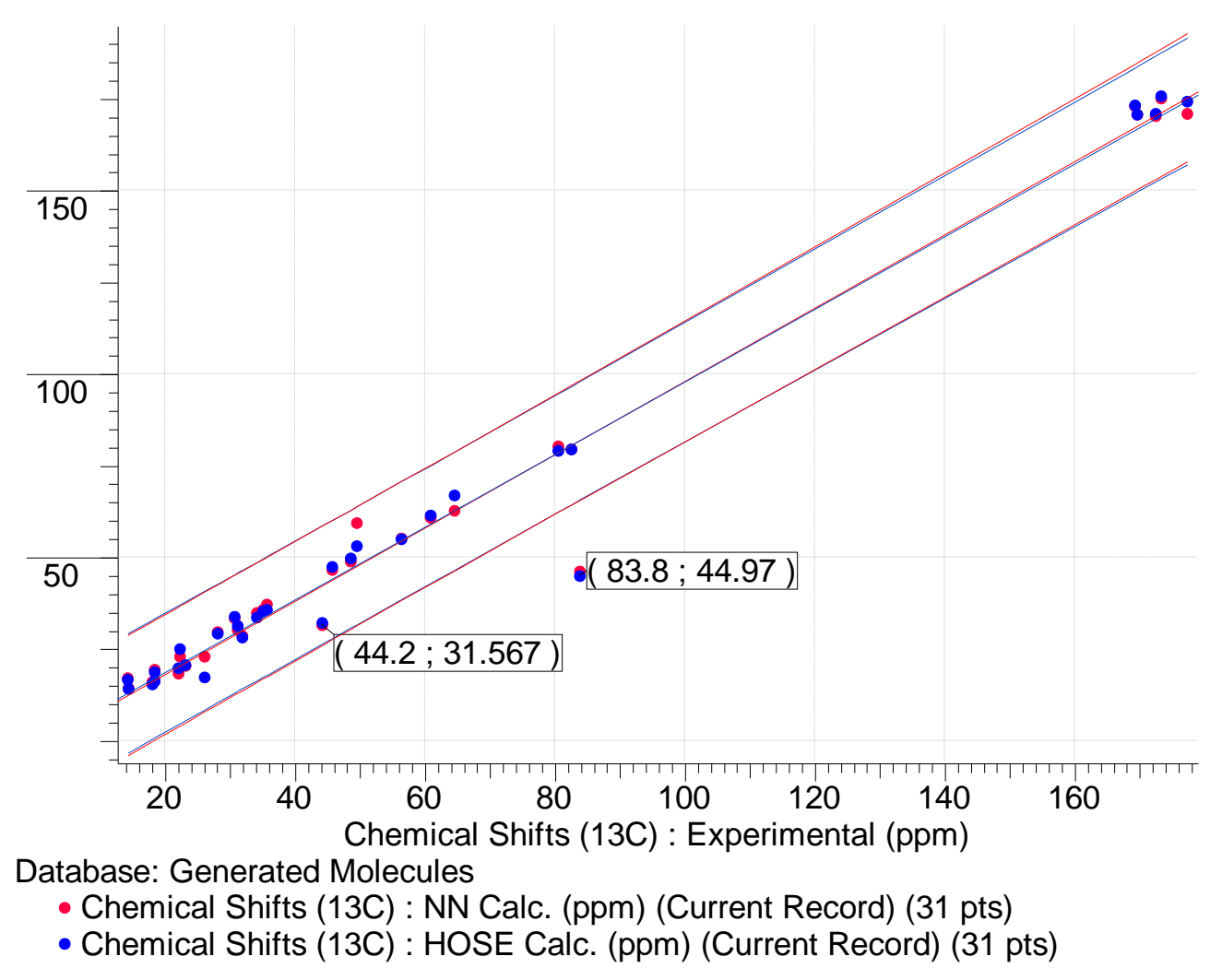

Figure 2. Linear regression plots for structure 2 generated from both HOSE and NN methods of ${ }^{13} \mathrm{C}$ chemical shift prediction. The first number shown in a box denotes the experimental chemical shift while the second is the calculated value. Both the HOSE and NN predictions practically coincide with the 45 -degree line $\left(\delta_{\text {calc }}=\delta_{\text {exp }}\right)$. Prediction limit lines are also shown. 


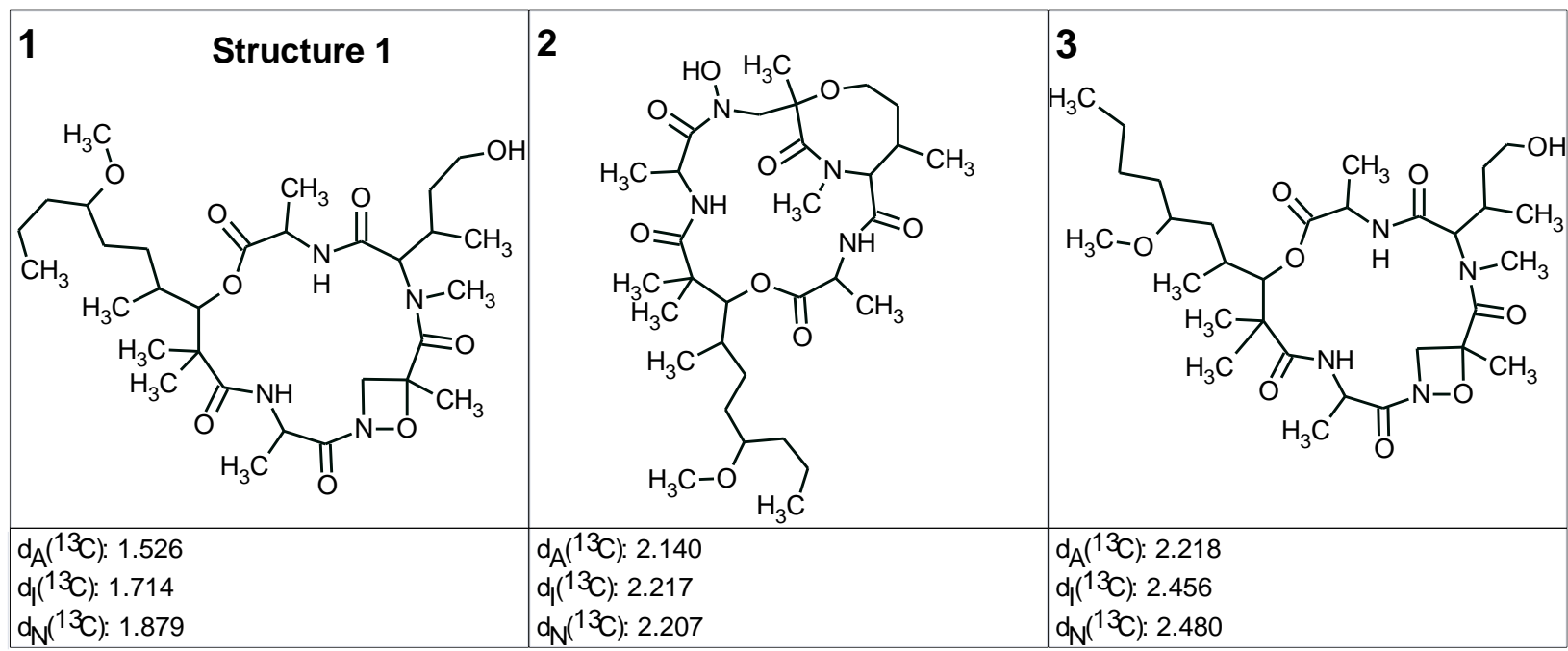

Figure 3. The first three structures of the ranked structural file when a molecular formula of $\mathrm{C}_{31} \mathrm{H}_{55} \mathrm{~N}_{4} \mathrm{O}_{9}$ was assumed. The numbers in the top left of each box correspond to the rank ordered structures.

In the next article ${ }^{44}$ by the same group of authors reported that using superior HRMS instrumentation capable of reaching a resolution of about 20000 they revised the molecular formula. A hint in regards to how to revise the structure was provided by the following finding: when a related natural product halipeptin $\mathrm{C}$ was isolated the presence of an unexpected sulfur atom in this compound was clearly detected by HRMS. The authors suggested that the molecule halipeptin A also contained a sulfur atom instead of two oxygen atoms to give a molecular formula of $\mathrm{C}_{31} \mathrm{H}_{54} \mathrm{~N}_{4} \mathrm{SO}_{7}$. In this case a pseudomolecular ion peak was found at $\mathrm{m} / \mathrm{z} 649.3628\left(\mathrm{M}+\mathrm{Na}^{+}, \Delta \mathrm{m}=-0.0017\right.$ or $\left.2.6 \mathrm{ppm}\right)$. For the original molecular formula $\mathrm{C}_{31} \mathrm{H}_{55} \mathrm{~N}_{4} \mathrm{O}_{9}$ the difference between the measured and calculated molecular mass was much higher: 0.0160 or $24.6 \mathrm{ppm}$, so the wrong hypothesis about the elemental composition would probably be rejected if a more precise $m / z$ value was obtained in the earlier investigation. With the revised molecular formula the following structure was deduced $^{44}$ : 


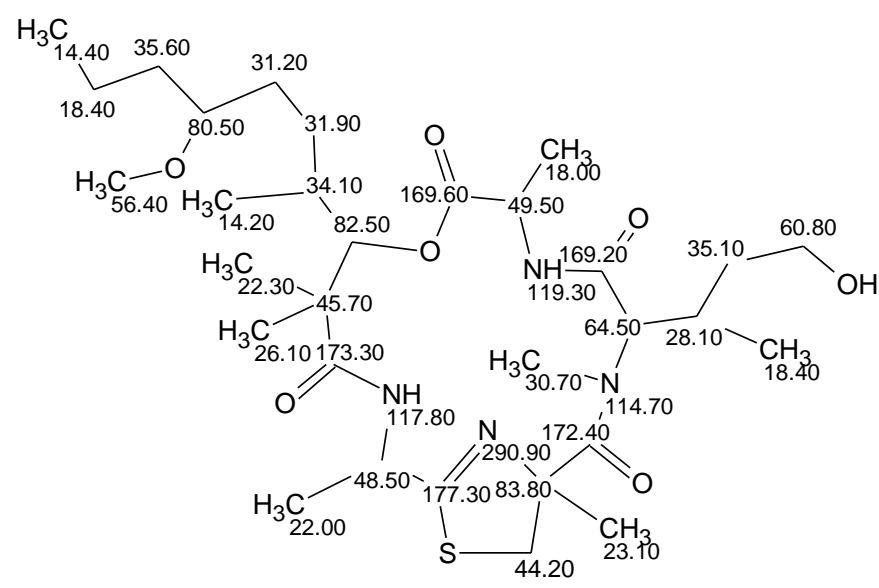

3

We will now show how this problem would be solved using the Structure Elucidator software. The accurate molecular mass of 627.4073 determined in reference ${ }^{41}$ was used as input for the molecular formula generator. Taking into account the number of signals in the ${ }^{13} \mathrm{C}$ NMR spectrum and the integrals in the ${ }^{1} \mathrm{H}$ NMR spectrum, the following admissible limits on atom numbers in molecular formula (the axioms of chemical composition) were set: $\mathrm{C}(31), \mathrm{H}(52-56), \mathrm{O}(0-10), \mathrm{N}(0-10), \mathrm{S}(0-2)$. For the initially determined mass of $627.4073 \pm 0.1$, three possible molecular formulae were generated: $\mathrm{C}_{31} \mathrm{H}_{54} \mathrm{~N}_{4} \mathrm{O}_{9}(\Delta \mathrm{m}=-$ 0.0104, $16.6 \mathrm{ppm}), \mathrm{C}_{31} \mathrm{H}_{54} \mathrm{~N}_{4} \mathrm{O}_{7} \mathrm{~S}_{1}(\Delta \mathrm{m}=-0.0281,44 \mathrm{ppm})$ and $\mathrm{C}_{31} \mathrm{H}_{54} \mathrm{~N}_{4} \mathrm{O}_{5} \mathrm{~S}_{2}(\Delta \mathrm{m}=-$ $0.0459,73 \mathrm{ppm})$ where the mass differences are shown in brackets. If high precision MS instruments are used then a mass difference exceeding $10 \mathrm{ppm}$ is commonly not acceptable. We suppose that in our case the value $\Delta \mathrm{m}=16 \mathrm{ppm}$ should suggest the presence of other elements or re-examination of the sample on a more advanced MS instrument.

We will show that if a CASE system is available correct structure elucidation of an unknown compound is possible even under non-ideal conditions. Though $\mathrm{C}_{31} \mathrm{H}_{54} \mathrm{~N}_{4} \mathrm{O}_{9}$ is obviously the most probable molecular formula based on the calculated mass defect the closest related formula, $\mathrm{C}_{31} \mathrm{H}_{54} \mathrm{~N}_{4} \mathrm{O}_{7} \mathrm{~S}_{1}$, can also be taken into account with the StrucEluc system.

Both the molecular formulae and the 2D NMR spectral data ${ }^{41}$ were used to perform structure generation with the same axioms listed earlier. The valence of the sulfur atom was set equal to 2 . An output file containing 303 structures was produced in 36 seconds. The three top structures of the output file ranked in ascending order of deviations are presented in Figure 4. The figure shows that the revised structure $\mathbf{3}$ is placed in first position by the program while the original structure is listed in second position. Application of the StrucEluc software would provide the correct structure from the molecular ion recorded even at modest resolution MS. This example also illustrates the methodology ${ }^{45}$ based on 
the application of an expert system which allows a user simultaneously to determine both the molecular and structural formula of an unknown compound.

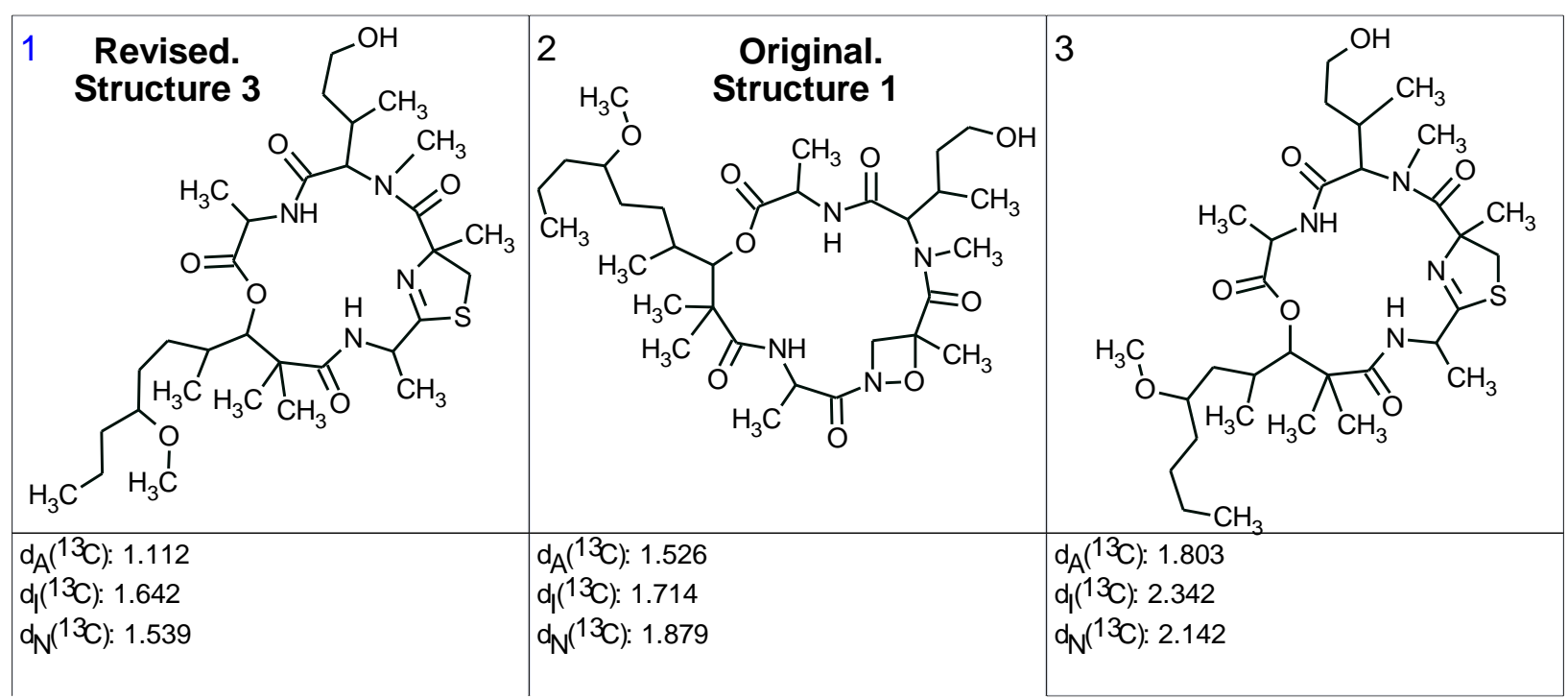

Figure 4. The top three structures of the output file generated from the two molecular formulae $\mathrm{C}_{31} \mathrm{H}_{54} \mathrm{~N}_{4} \mathrm{O}_{9}$ and $\mathrm{C}_{31} \mathrm{H}_{54} \mathrm{~N}_{4} \mathrm{O}_{7} \mathrm{~S}_{1}$. The numbers in the top left of each box correspond to the rank ordered structures.

For clarity the differences between the original and revised structures are shown in Figure 5.

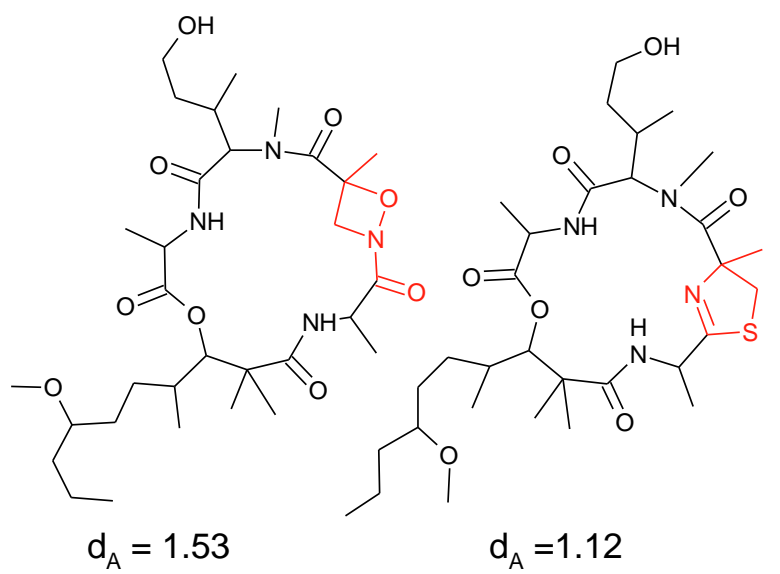

Figure 5. The original and revised structures of halipeptin A.

Sakuno et al $^{46}$ isolated an aflatoxin biosynthesis enzyme inhibitor with molecular formula $\mathrm{C}_{20} \mathrm{H}_{18} \mathrm{O}_{6}$. It is labeled as TAEMC161. The following structure for this alkaloid was suggested from the 1D NMR, HMBC and NOE data (an experimental chemical shift assignment suggested by authors is displayed): 


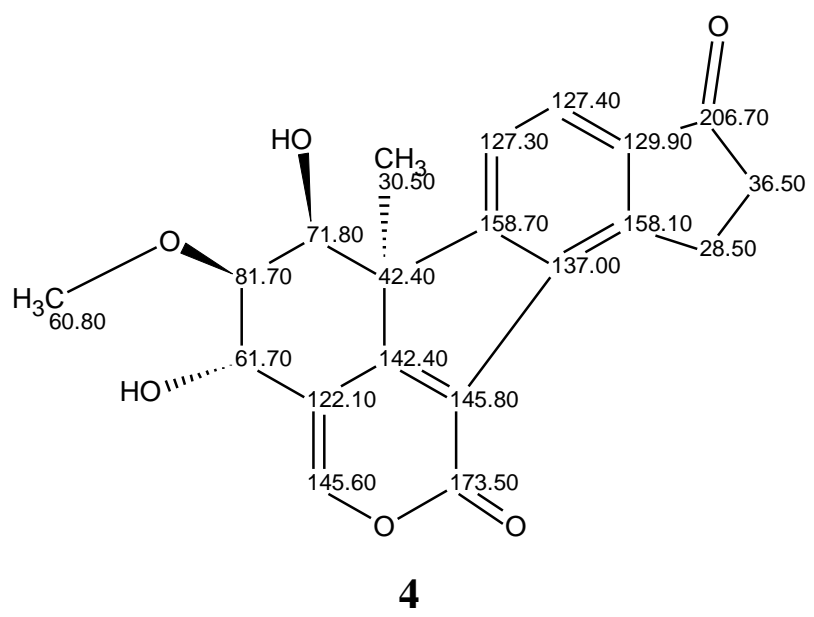

During the process of structure elucidation the authors ${ }^{46}$ postulated that the ${ }^{13} \mathrm{C}$ chemical shift at $173.50 \mathrm{ppm}$ was associated with the resonance of the ester group carbon. The spectral data were input into the StrucEluc system and, similar to Sakuno et al $\mathrm{O}=\mathrm{C}-\mathrm{O}$ group was involved in the process of fuzzy structure generation by manually adding to the molecular connectivity diagram (MCD). The results gave: $\mathrm{k}=174 \rightarrow 80 \rightarrow 60, t_{g}=30 \mathrm{~s}$. When the output file was ordered as described above then structure 4 occupied first position but with deviation values of about 4.5 ppm. Such large deviations suggest caution ${ }^{26-28}$ and close inspection of the data. It should be remembered that the accuracy of chemical shift calculation is about 1.6-1.8 ppm.

Wipf and Kerekes ${ }^{47}$ compared the NMR and IR spectra of TAEMC161 with a number of spectra of its structural relatives and found close similarity between the spectra of TAEMC161 and viridol, 5:

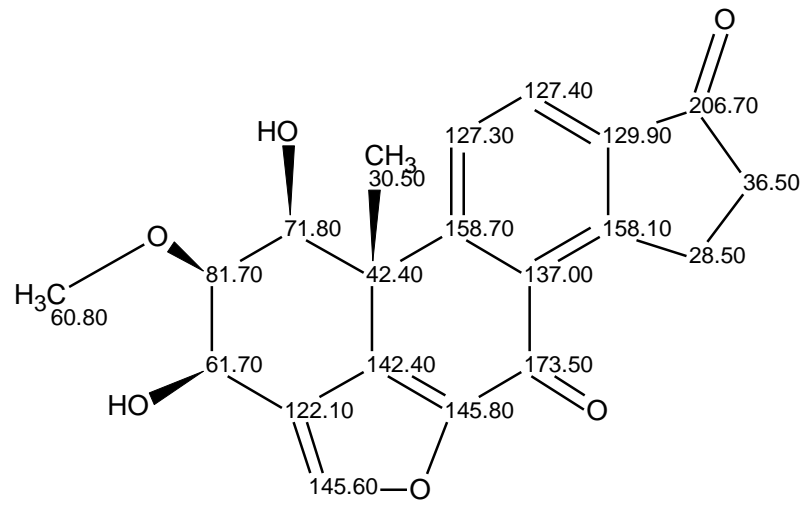

5

In this molecule both carbonyl groups are ketones and the structure is in accordance with the 2D NMR data used for deducing structure 4. Density functional theory calculations of ${ }^{13} \mathrm{C}$ chemical shifts were performed by authors ${ }^{47}$ for structures $\mathbf{4}$ and $\mathbf{5}$ using GIAO approximation. It was proven that TAEMC161 is actually identical to $\mathbf{5}$. We repeated structure generation without any constraints imposed on the carbonyl groups with the following result: $k=494 \rightarrow 398 \rightarrow 272, t_{g}=1 \mathrm{~m}$ $40 \mathrm{~s}$. The three top structures in the ranked output file are presented in Figure 6. 


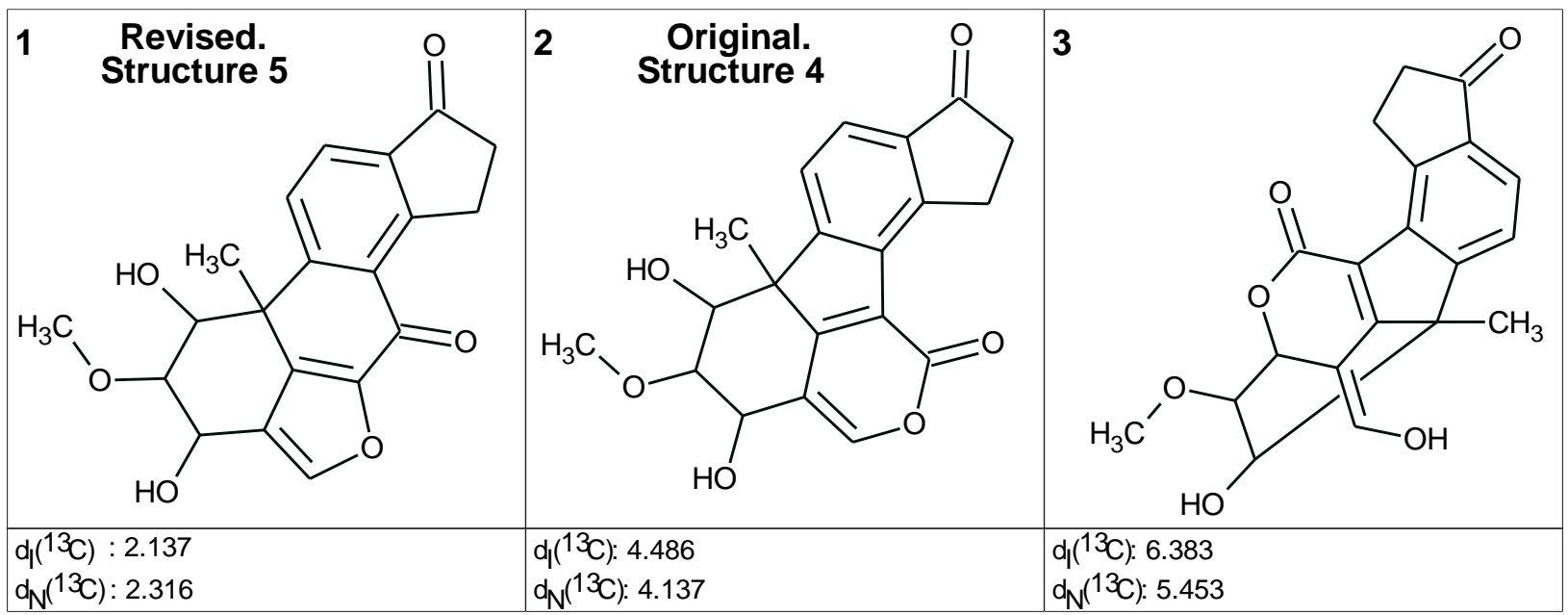

Figure 6. The top three structures of the output file generated for compound $\mathrm{C}_{20} \mathrm{H}_{18} \mathrm{O}_{6}$ (viridol).

The numbers in the top left of each box correspond to the rank ordered structures.

The figure shows that empirical prediction of ${ }^{13} \mathrm{C}$ chemical shifts convincingly demonstrates the superiority of the revised structure over the original suggested for TAEMC161. The differences between the original and revised structures are shown in Figure 7

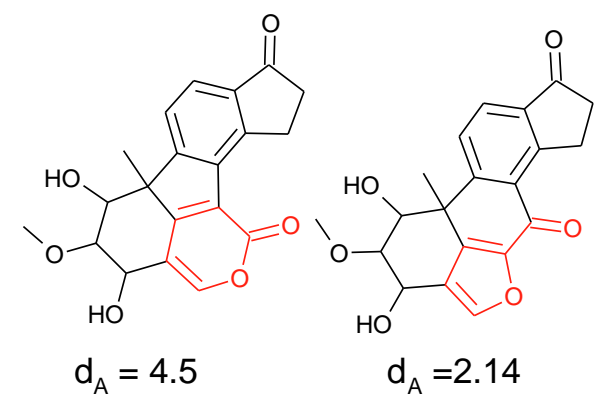

Figure 7. The original and revised structures of inhibitor (viridol).

In 1997 Cóbar et al ${ }^{48}$ isolated three new diterpenoid hexose-glycosides, calyculaglycodides $\mathrm{A}, \mathrm{B}$ and $\mathrm{C}$, and their structures were determined from MS, $1 \mathrm{D}$ NMR , COSY, ${ }^{1} \mathrm{H}-{ }^{13} \mathrm{C} \mathrm{HMBC}$ and NOE spectra. The following structure was suggested for calyculaglycodide B (molecular formula $\left.\mathrm{C}_{30} \mathrm{H}_{48} \mathrm{O}_{8}\right)$ :

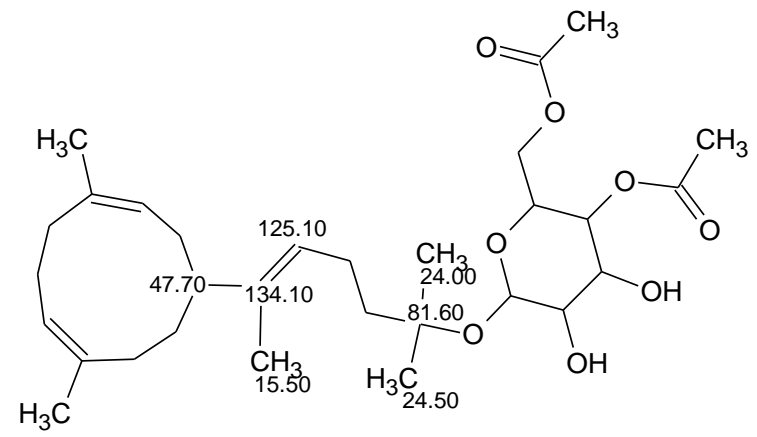


In 2001 the same group ${ }^{49}$ reinvestigated this natural product and discovered that structure 6 is incorrect. A hint to revision of the structure was obtained on the basis of the comparison of NMR spectra of similar compounds which were isolated from the same material. It was noticed that the NMR spectra of all compounds including an aglycone substructure contained indistinguishable portions of the spectra. With this in mind, the NMR and mass spectra of calyculaglycodides A, B and C were thoroughly reinvestigated and as a result the revised structure 7 was postulated for calyculaglycodide $B$ :

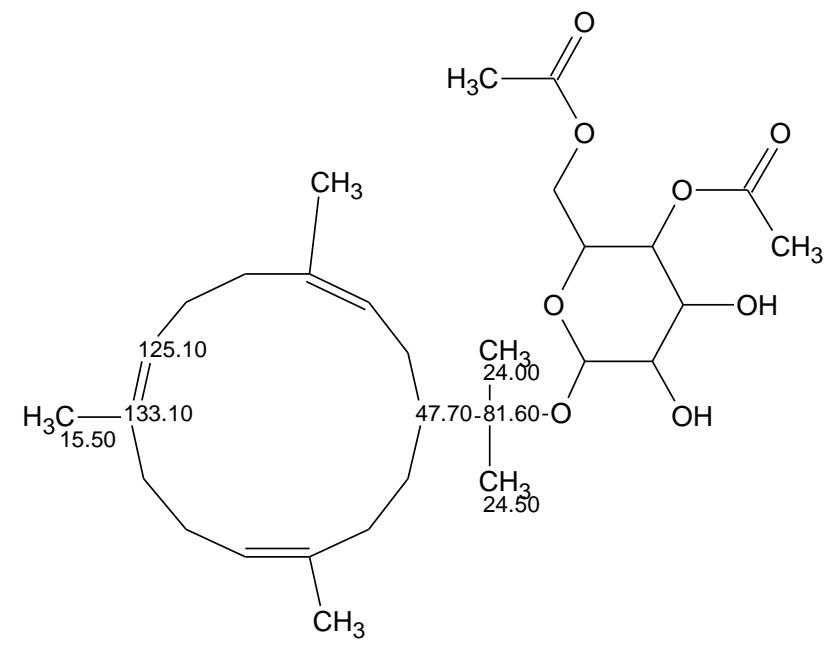

7

Freshly recorded NMR spectra showed that the $\mathrm{HMBC}$ connectivity $\mathrm{CH}_{3}(15.5) \rightarrow \mathrm{C}(47.7)$ was earlier identified as an artifact while a strong correlation of the dimethyl group to $\mathrm{C}(47.5)$ was missed. As a consequence the initial set of axioms was false and inferring the correct structure was absolutely impossible. The ${ }^{13} \mathrm{C}$ chemical shifts predicted for structure $\mathbf{6}$ led to average deviations of values around $2 \mathrm{ppm}$ which are of an appropriate magnitude to not further question the correctness of structure.

When the corrected HMBC data were input into StrucEluc the program detected the presence of NSCs, and FSG was carried out. During fuzzy generation the program determined that there were 2 NSCs and provided the following results: $\mathrm{k}=10 \rightarrow 6, t_{g}=1 \mathrm{~h} 39 \mathrm{~m}$. The time of structure generation is quite long because in this case the program tried to generate structures from 861 different combinations of connectivities (see Section 3). The revised structure was selected using ${ }^{13} \mathrm{C}$ spectral prediction to choose the most probable one (see Figure 8). The difference between the structures is only in the positions of the double bond and methyl group on the large cycle (see Figure 9). 


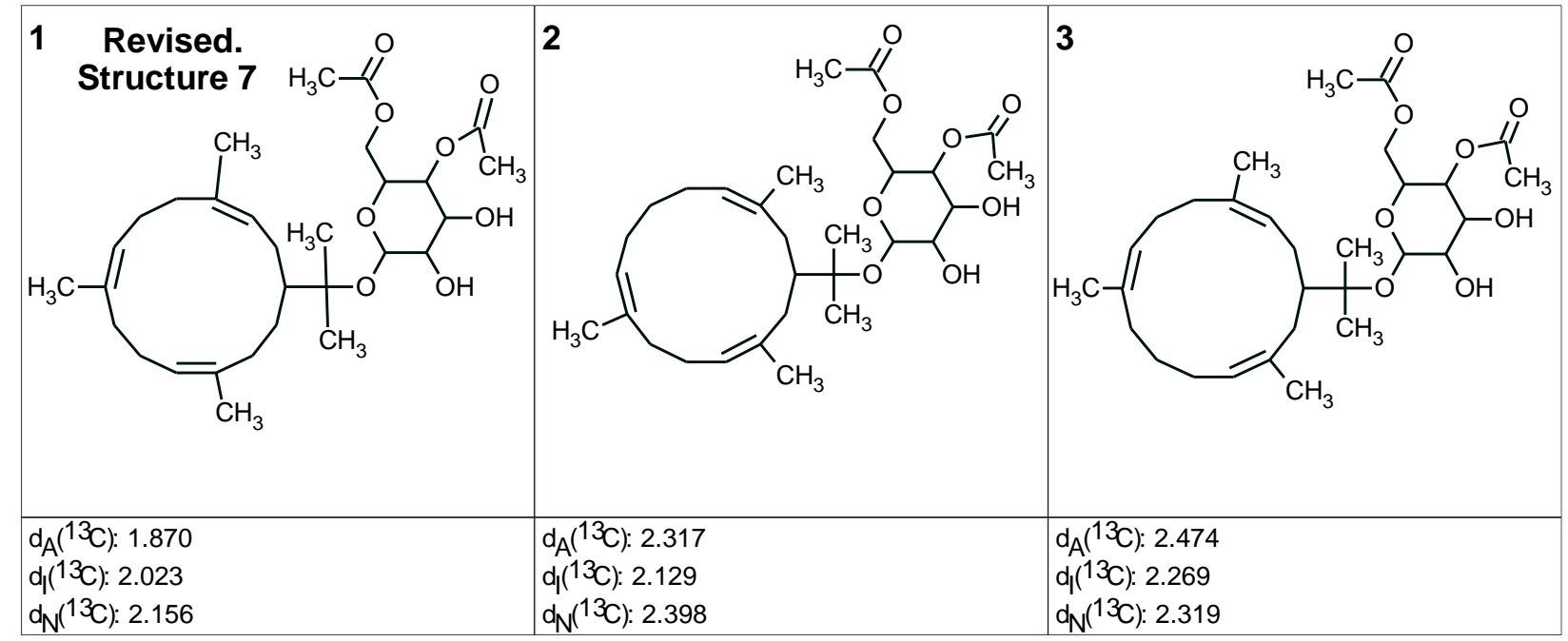

Figure 8. The top structures of the output file generated by the StrucEluc software for the $\mathrm{C}_{30} \mathrm{H}_{48} \mathrm{O}_{8}$ compound calyculaglycodide $B$.
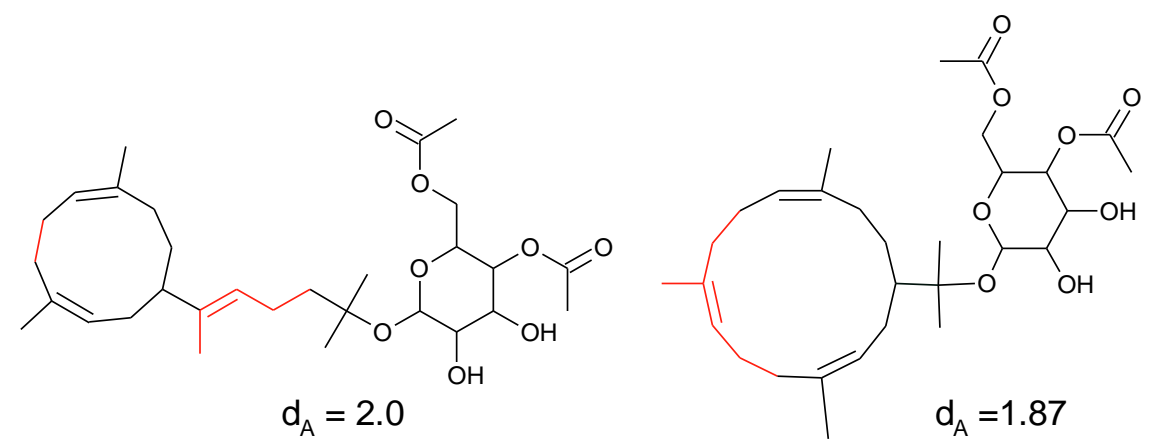

Figure 9. The original and revised structures of calyculaglycodide $\mathrm{B}$

Ralifo and Crews ${ }^{50}$ reported on the separation (an isolated amount of about $3.2 \mathrm{mg}$ ) of (-)spiroleucettadine $8\left(\mathrm{C}_{20} \mathrm{H}_{23} \mathrm{~N}_{3} \mathrm{O}_{4}\right)$, the first natural product to contain a fused 2-aminoimidazole oxalane ring. In spite of the modest size of this molecule the high value of the double bond equivalent $(\mathrm{DBE}=11)$ hints that the structure elucidation may be a very complicated problem.

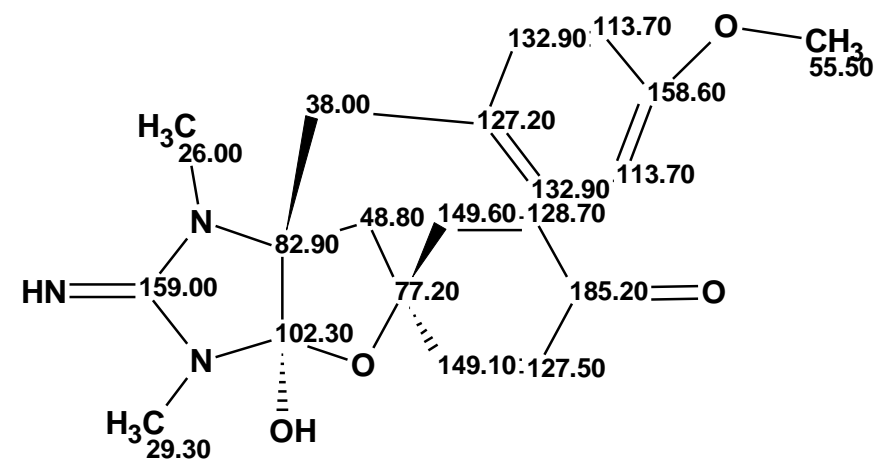

The structure was inferred on the basis of the 2D NMR data, as well as by structural and spectral comparison between structure $\mathbf{8}$ and a series of known molecules of similar structure and 
origin. The authors ${ }^{50}$ suggested the presence of a guanidine group ( $\delta \mathrm{C} 159.0$ ) substituted with two methyls (axiom 1). This proposition was justified based on the characteristic $\mathrm{NCH}_{3}$ signals at $(29.3 ; 2.48)$ and $(26.0 ; 2.91)$, along with the gHMBC correlation from $\mathrm{NCH}_{3}(2.48)$ to $\mathrm{C}(159)$. The absence of an expected $\mathrm{HMBC}$ correlation from $\mathrm{NCH}_{3}(2.91)$ to $\mathrm{C}(159.0)$ was considered as acceptable and the possible reason for the absence of the correlation was not analyzed. The position of carbon $\mathrm{C}(48.8)$ was confirmed by a HMBC correlation from this nucleus to the hydrogen $\delta \mathrm{H}(1.97)$ attached to $\mathrm{C}(38.0)$ (axiom 2). The signal of exchangeable hydrogen in the ${ }^{1} \mathrm{H}$ NMR spectrum was assigned to an $\mathrm{OH}$ group (axiom 3) but no attempt to confirm this postulate by IR spectroscopy was mentioned in the article. The relative stereochemistry of structure $\mathbf{8}$ was determined using a combination of ROESY data and molecular modeling. The absolute stereochemistry was determined using OED-CD spectroscopy.

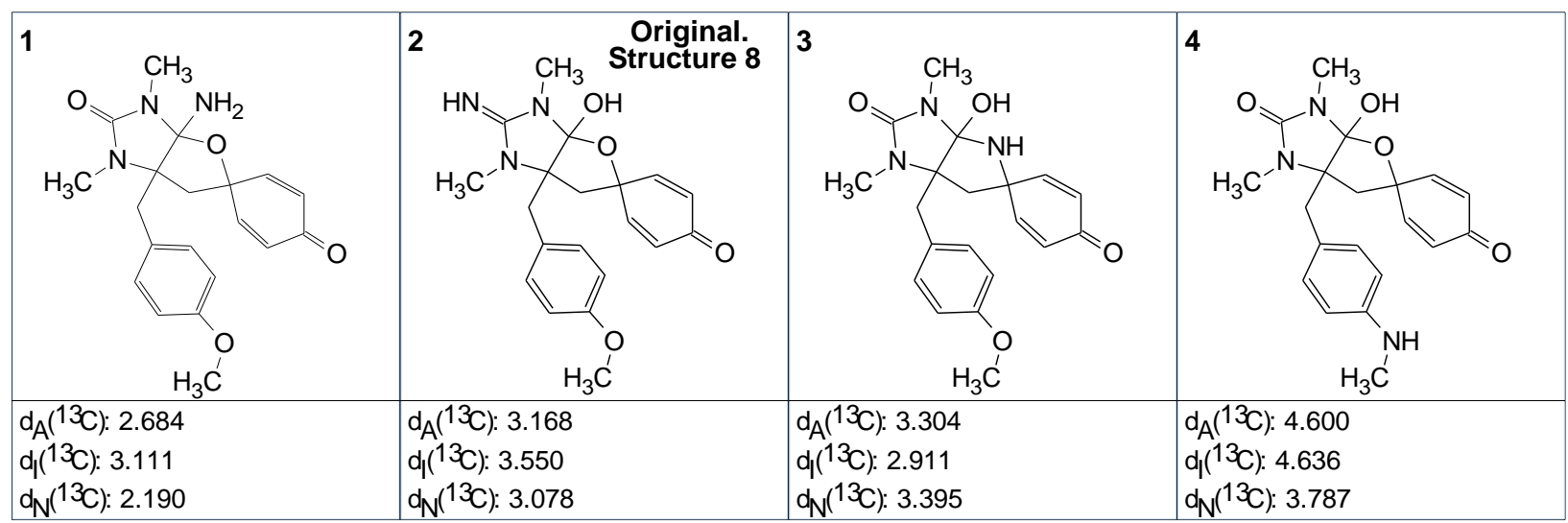

Figure 10. The top ranked structures of the output file generated by the StrucEluc software for the $\mathrm{C}_{20} \mathrm{H}_{23} \mathrm{~N}_{3} \mathrm{O}_{4}$ compound (-)-spiroleucettadine elucidated from the 2D NMR data contained in ref. ${ }^{50}$ The numbers in the top left of each box correspond to the rank ordered structures.

As a result of utilizing the $1 \mathrm{D}$ and HMBC NMR data published by the authors ${ }^{50}$ as an input to the StrucEluc system the following result was obtained under the conditions of strict structure generation: $\mathrm{k}=117 \rightarrow 83 \rightarrow 79, t_{g}=10 \mathrm{~s}$. Figure 10 presents the best ranked structures from the start of the output file. Note that structures containing too "exotic" fragments were deleted. The postulated axioms led to a preferred structure that differs from the original structure $\mathbf{8}$ which was also generated: instead of the $\mathrm{C}=\mathrm{NH}$ fragment this structure contains a $\mathrm{C}=\mathrm{O}$ group while the $\mathrm{OH}$ group is replaced by an $\mathrm{NH}_{2}$ group. The third and fourth structures also contain a carbonyl group at the same position. There is no doubt that if the computer-based solution presented in Figure 10 was available to Crews's group, one of the leading groups in the chemistry of natural products, then their elucidated structure for $\mathbf{8}$ would be questioned and a different and likely correct structure would be found after appropriate revision of the experimental data and set of axioms. 
Structure $\mathbf{8}$ was met with keen interest by the natural products and synthetic communities and several attempts to synthesize it were undertaken but without any success. Questions regarding the original structure elucidation process therefore arose. Aberle et $a l^{51}$ suggested structures 9 and $\mathbf{1 0}$ as alternatives but DFT calculations of chemical shifts performed by the Crews's group ${ }^{52}$ showed that both of them should be declined.

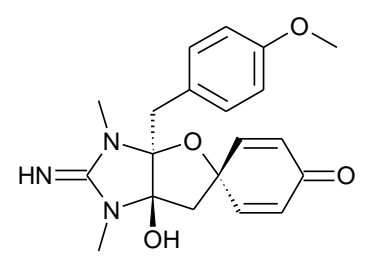

9

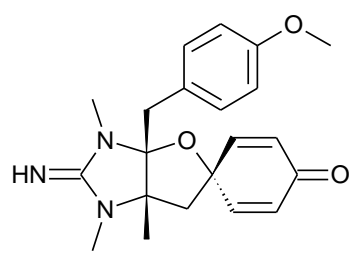

10

With this in mind the Crews's group ${ }^{52}$ fulfilled a successful re-isolation of spiroleucettadine, and X-ray analysis established the correct structure of spiroleucettadine, shown as $\mathbf{1 1}$ below.

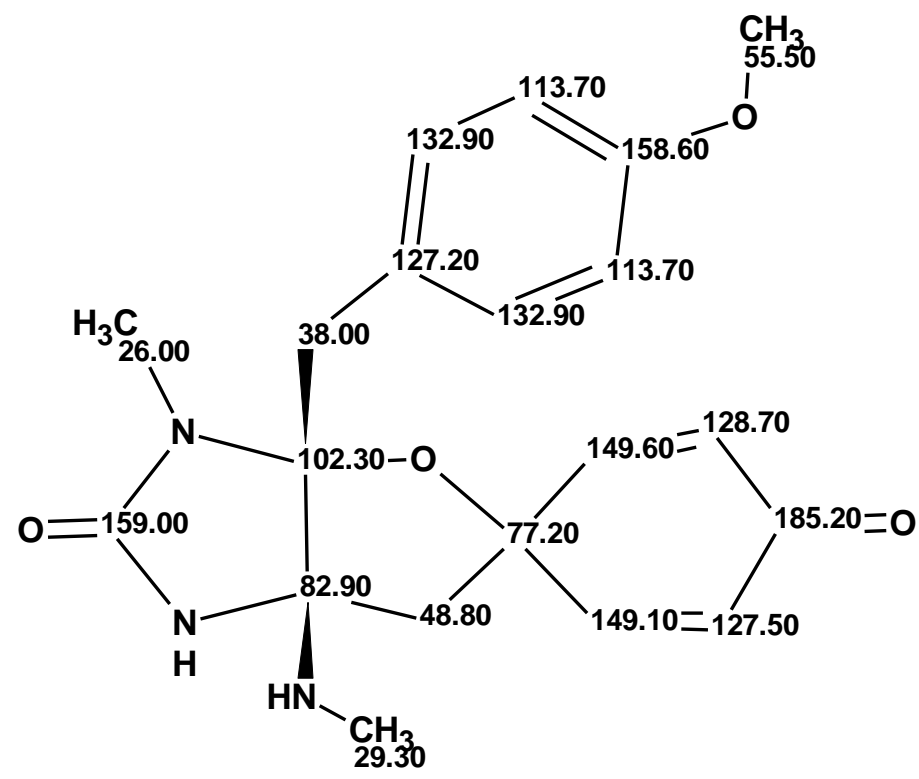

Fresh 2D NMR data on spiroleucettadine were obtained and verified ${ }^{52}$. It was revealed that the connectivity from $\mathrm{C} 48.8$ to $\mathrm{C} 38.0$ for structure $\mathbf{8}$ in methanol- $d_{4}$ was actually due to a solvent $J_{\mathrm{CH}}$ peak. In this case axiom 2 was false. An inconsistency in axiom 1 became evident due to the lack of parity displayed between the two $N$-methyl groups as follows from structure 11. The relative stereochemistry was also revised as shown in structure $\mathbf{1 1}$ and its superiority over structures 8-10 was proven by DFT calculations.

When the new 2D NMR data were input into the StrucEluc system the structure generation was performed with very "liberal" atom properties: no constraints for heteroatom neighboring for carbons with chemical shifts in the interval range of 113.7-158.6 ppm. The following solution 
was obtained: $\mathrm{k}=342 \rightarrow 256, t_{g}=8 \mathrm{~h} 2 \mathrm{~m}$. The reason for the long generation time, the so-called "overnight mode", was the high DBE value and the lack of structural restrictions. The best structures are presented in Figure 11.

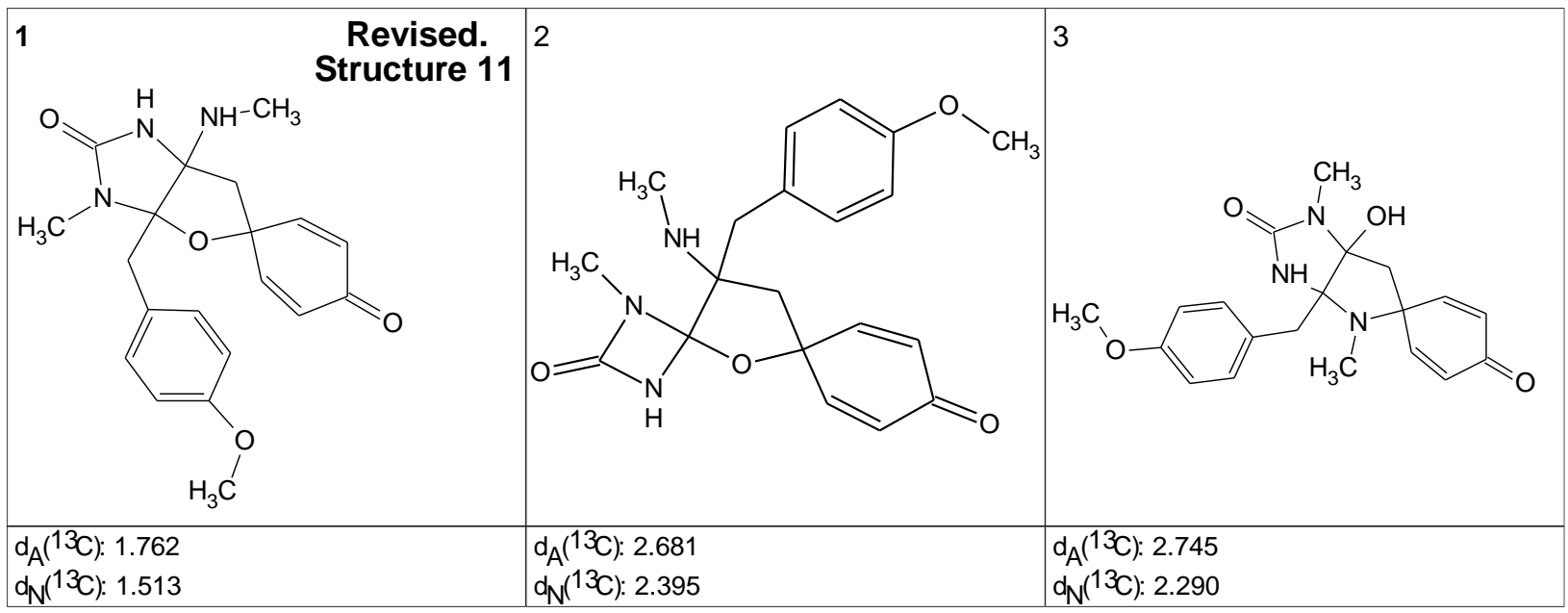

Figure 11. The highest ranked structures of the output file generated by the StrucEluc software for the $\mathrm{C}_{20} \mathrm{H}_{23} \mathrm{~N}_{3} \mathrm{O}_{4}$ compound from the new 2D NMR data obtained from ref. ${ }^{52}$ The numbers in the top left of each box correspond to the rank ordered structures.

The revised structure 11 was selected as the most probable one by the program in accord with the results of crystallographic analysis and the conclusions of the researchers ${ }^{52}$. The differences between the original and revised structures are shown in Figure 12.

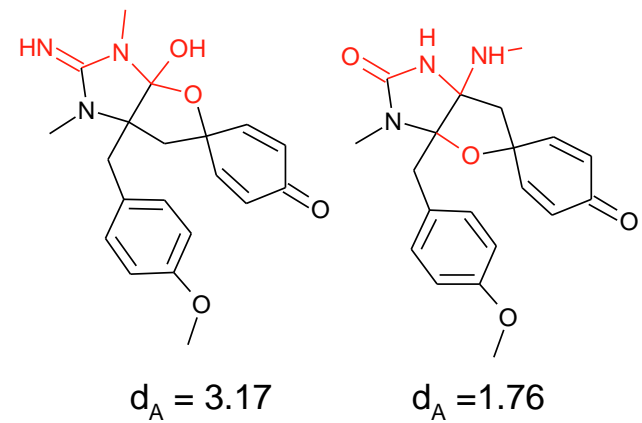

Figure 12. The original and revised structures of (-)-spiroleucettadine

Since four isomeric structures (8-10) and the first ranked structure in Figure 10 were considered as potential candidates for the genuine structure the authors ${ }^{52}$ carried out DFTbased ${ }^{13} \mathrm{C}$ chemical shift calculations using the B3LYP/6-31G*//B3LYP/6-31G* protocol for all stereoisomers. This resulted in the examination of a total of 16 structures and their modifications where the oxygen atom in the 5-membered ring was migrated either "up and down". It was found that the configuration of structure $\mathbf{1 1}$ corresponds to the minimum 
discrepancy between the experimental and calculated spectra, while structure $\mathbf{1 0}$ got a low rank.

We performed ${ }^{13} \mathrm{C}$ chemical shift prediction using HOSE code-based and neural net algorithms ${ }^{35,43}$ for the same structure set (see Table 1). Note that both methods take stereochemistry into account (see Section 3). As a result stereoisomer 11 was also distinguished as the best by empirical calculations. The total elapsed time was 7 min with no geometry optimization being necessary.

Table 1. Selection of the correct structure and the best stereoisomer of spiroleucettadine.

Structures are labeled as in ref ${ }^{52}$.

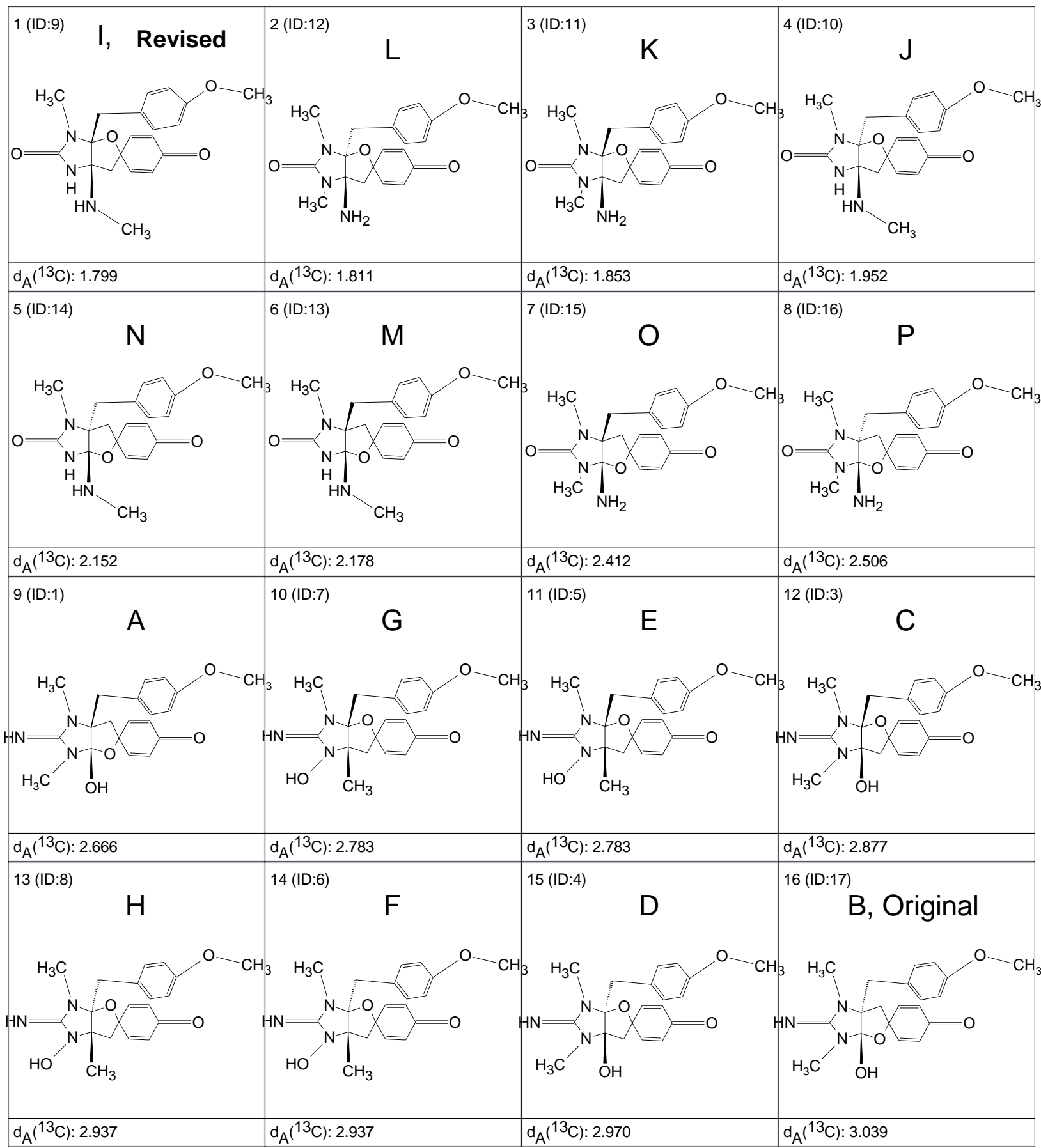


Buske et l $^{53}$ described the structural elucidation of antidesmone, 12, a novel type tetrahydroisoquinoline alkaloid with molecular formula $\mathrm{C}_{19} \mathrm{H}_{29} \mathrm{NO}_{3}$ :

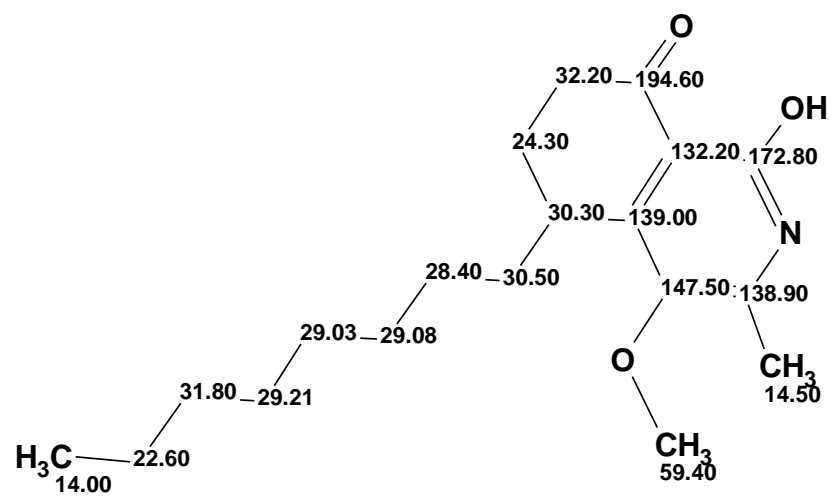

12

Antidesmone was identified as an unprecedented and novel alkaloid where the nitrogen is located in the aromatic ring and the substitution pattern, in particular the unusual n-octyl residue on the isocyclic ring, is also unique. The authors ${ }^{53}$ reported that no HMBC correlations to carbon 172.8 could be found but from the chemical shift and molecular formula they deduced the presence of an $\mathrm{OH}$ group attached to this carbon. This axiom crucially influenced the solution of the problem. The absolute configuration of antidesmone was determined using its methyl ether for which quantum chemical calculations of CD and UV spectra were performed.

The NMR data presented ${ }^{53}$ were used to determine which structure would be deduced by StrucEluc from the published spectral data as the best structure if the assumptions of the researchers were included into the initial data of the program. The attachment of an $\mathrm{OH}$ group at carbon 172.8 was accepted as an axiom. The first run was performed in strict generation mode with the result $k=13092 \rightarrow 12636 \rightarrow 1031, t_{g}=1 \mathrm{~m} 13 \mathrm{~s}$. The first ranked structure gave deviations with values between 3.5-4.7 $\mathrm{ppm}$. This hinted at the presence of at least one NSC. At the same time structure 12 was not generated. Fuzzy Structure Generation was initiated with the following result: $\mathrm{k}=144228 \rightarrow 116496 \rightarrow 6604, t_{g}=19 \mathrm{~m}$ 28 s. The best structure was identical to that in the previous run but structure 12 was generated this time and ranked in 113th position by neural network based chemical shift calculation. This is very convincing that structure $\mathbf{1 2}$ is incorrect. It is obvious that some incorrect restrictions (axioms) were included into the initial set of statements.

The problem was solved using StrucEluc to analyze the 2D NMR data. Our common methodology was used: no user defined constraints were imposed on the generated structures and the fragment $=\mathrm{C}-\mathrm{O}-\mathrm{H}$ remained disconnected in the MCD. Strict structure 
generation gave the following result: $\mathrm{k}=59916 \rightarrow 51888 \rightarrow 4274, t_{g}=6 \mathrm{~m} 5 \mathrm{~s}$. Chemical shift calculations using all three methods promoted the structure $\mathbf{1 3}$ to first position in the ranked output file with the following average deviations: $d_{A}=1.437, d_{N}=2.767, d_{I}=1.964$.

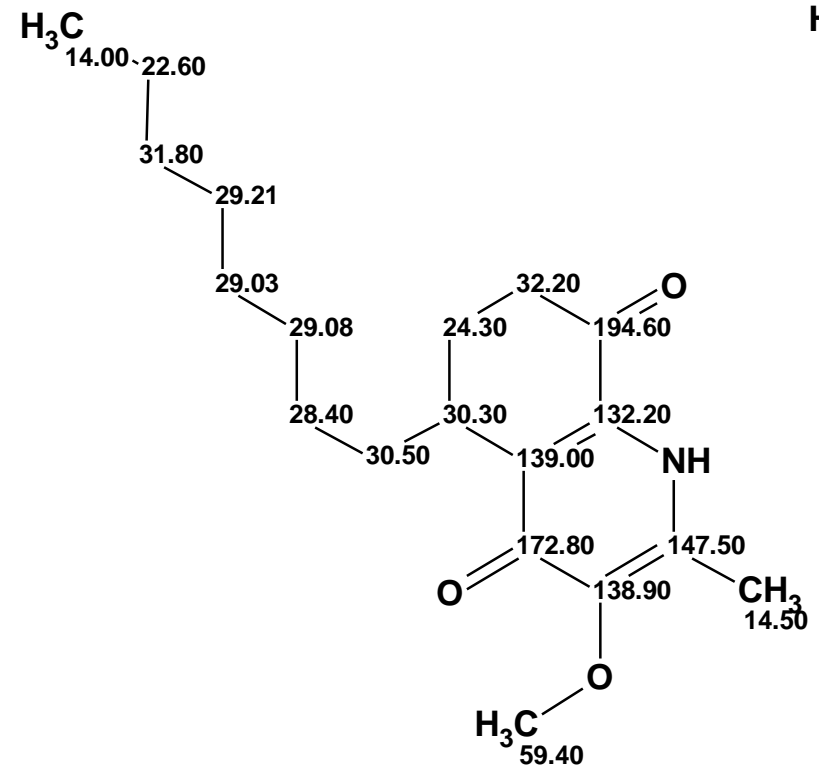

13

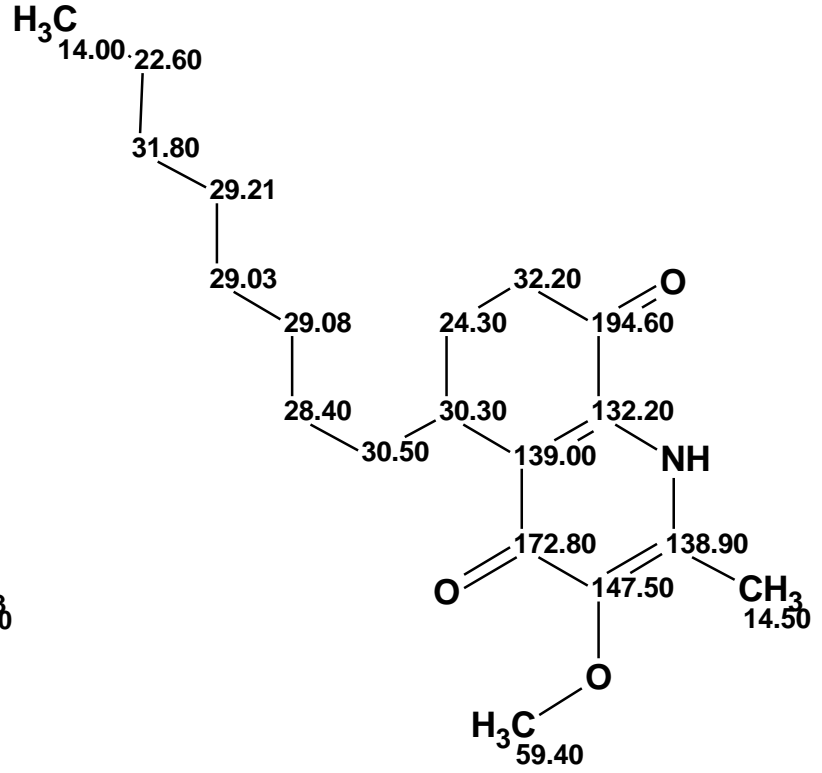

13A

Structure 12 was also generated but it was ranked 342nd by NN prediction and 183rd using HOSE-based prediction. Application of StrucEluc allowed us to establish the most probable structure and reject the author's ${ }^{53}$ original structural suggestion.

In the next article published by the same group ${ }^{54}$ it was reported that structure 12 was mistaken due to the poor quality of the 2D NMR spectral data obtained from a small amount of sample. The correct structure, 13, was inferred for antidesmone from fresh 2D NMR data including HSQC, HMBC, COSY and NOESY. When the new HMBC data were used as input for the StrucEluc system the program produced the following results: $k=3972$ $\rightarrow 3876 \rightarrow 323, t_{g}=1 \mathrm{~m} 13 \mathrm{~s}$. The best structure 13A $\left(d_{A}=0.974, d_{N}=2.056, d_{I}=1.572\right)$ coincided with structure 13, but the chemical shift assignment was refined according to the improved 2D NMR data and the chemical shifts at 147.5 and 138.9 were exchanged. For clarity the differences between the original and revised structures are shown in Figure 13

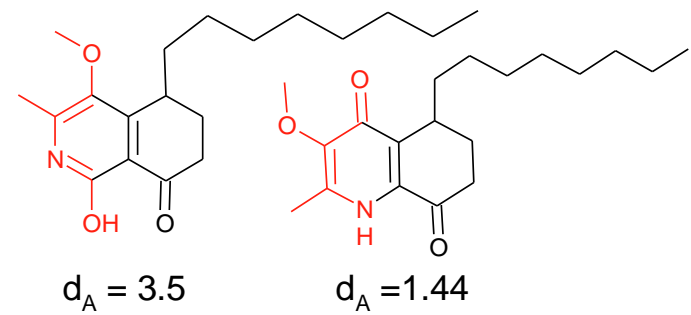

Figure 13. The original and revised structures of antidesmone 
This example shows that even in those cases when the spectral data are of low quality the correct structure can still be determined in certain cases. It was possible because when the StrucEluc system is utilized the chemist can afford to avoid subjective suggestions such as those postulated by the authors ${ }^{53}$.

\subsection{Revision of structures with application of chemical synthesis.}

In 2004 Hsieh et $a l^{55}$ isolated a new alkaloid with molecular formula $\mathrm{C}_{15} \mathrm{H}_{10} \mathrm{~N}_{2} \mathrm{O}_{2}$ (DBE=12) and named as drymarietin (5-methoxycanthin-4-one). Using a combination of ${ }^{1} \mathrm{H}-{ }^{13} \mathrm{C}$ HMBC and ${ }^{1} \mathrm{H}^{15}{ }^{15} \mathrm{HMBC} 2 \mathrm{D}$ NMR data they hypothesized the drymarietin structure to be as shown in $\mathbf{1 4}$ with the chemical shift assignment shown.

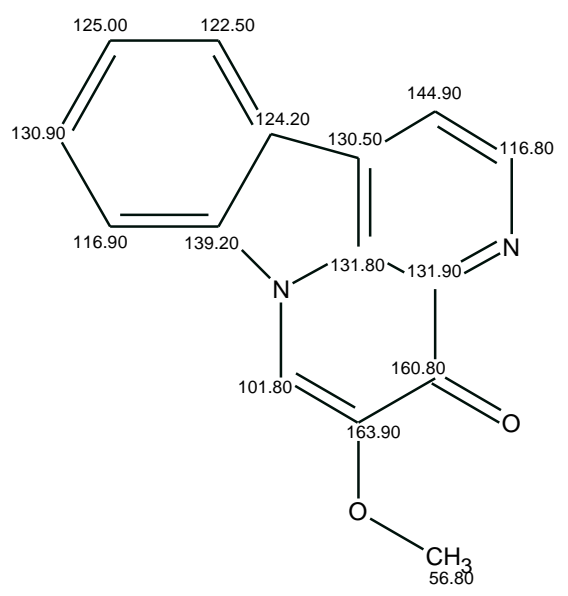

14

This alkaloid showed interesting anti-HIV activity and has been mentioned in a series of review articles dealing with bioactive natural products ${ }^{56}$.

In 2009 Wetzel et $a l^{56}$ revised structure 14. They synthesized 5-methoxycanthin-4-one and discovered that the synthetic product displayed spectroscopic data significantly different from those of the drymarietin alkaloid. Extensive re-evaluation of the spectroscopic data published for this and related alkaloids led them to the conclusion that drymarietin is identical to the known alkaloid cordatanine 15 (4-methoxycanthin-6-one):

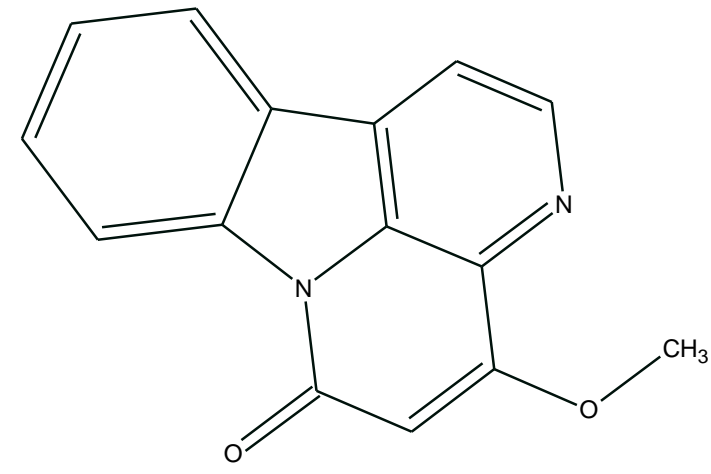


To investigate whether CASE methods could help researchers to avoid a pitfall in this case, we first predicted the ${ }^{13} \mathrm{C}$ chemical shifts of structure $\mathbf{1 4}$ and determined that all average deviations were 8-9 ppm. This unambiguously demonstrated that the structure does not correspond to the ${ }^{13} \mathrm{C}$ NMR spectrum. The calculated shifts are shown in structure $14 \mathrm{~A}$ where the shifts with the largest differences are marked in bold:

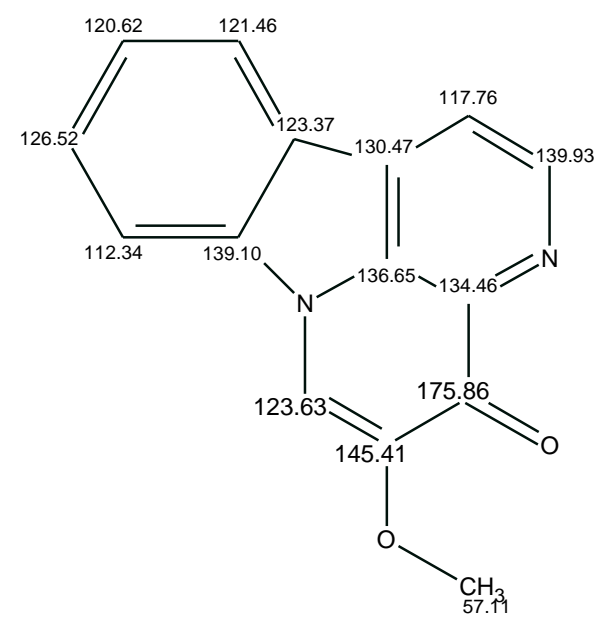

14A

Figure 14 shows a linear regression plot for the experimental versus calculated shifts for structure 14. 


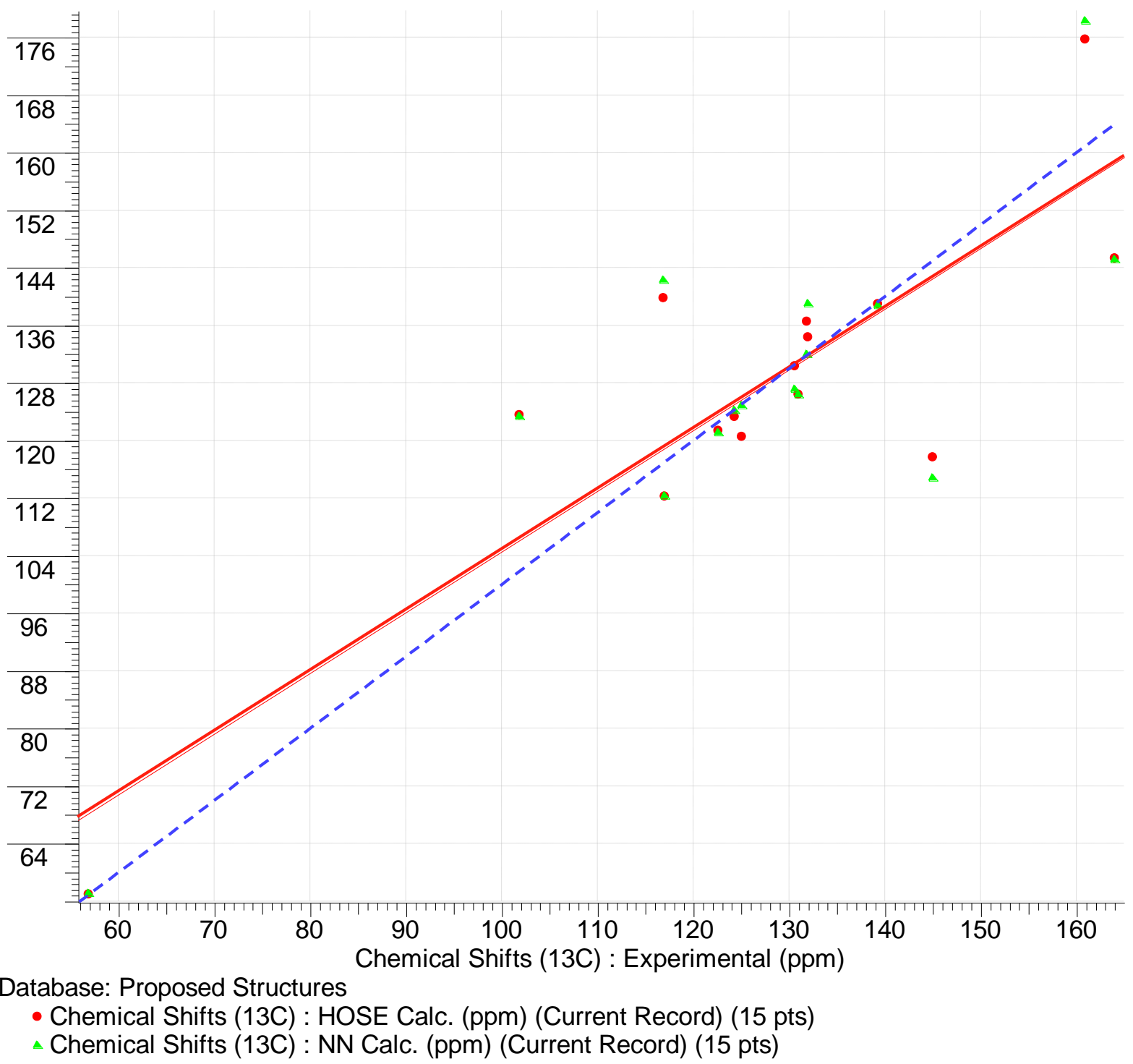

Figure 14 Linear regression plots for structure 14 generated using both HOSE and NN methods of ${ }^{13} \mathrm{C}$ chemical shift prediction. The linear regression parameters are: $\mathrm{R}^{2}(\mathrm{HOSE})=0.742, \delta_{\mathrm{HOSE}}$ $=0.843 \delta_{\exp }+20.3 ; \mathrm{R}^{2}(\mathrm{NN})=0.710, \delta_{\mathrm{NN}}=0.841 \delta_{\exp }+20.9$. The intersection angle between the regression plot and the 45 -degree line is equal to $-4^{\circ}$.

Figure 14 is convincing evidence that the structure and chemical shift assignment are wrong. We posited the question what structure would be inferred by the StrucEluc program if the data of Hsieh et al were used as input for the system?

The program created an MCD which clearly showed the presence of a benzene ring. The corresponding atoms were therefore connected by chemical bonds. Structure generation quickly identified the presence of 3 NSCs in the 2D NMR data and Fuzzy Structure Generation performed using $m=3$ and $a=1$ ( $a$ is the number of bonds by which the connectivity length should be augmented) gave the following result: $\mathrm{k}=3149 \rightarrow 1463 \rightarrow 146, t_{g}=56 \mathrm{~s}$. 
The best ranked structures are presented in Figure 15 where correct structure $\mathbf{1 5}$ was ranked first. Application of ${ }^{13} \mathrm{C}$ spectrum prediction therefore showed that structure $\mathbf{1 6}$ was wrong. The correct solution 15 was then obtained without any synthesis of the suggested structure 14. If the authors ${ }^{55}$ had used fast ${ }^{13} \mathrm{C}$ chemical shift prediction to verify their hypothesis (structure 14) then it would allow them to detect the wrong structural suggestion. In this case no chemical synthesis would be necessary to disprove structure 14.

Structure 14 which was synthesized by Wetzel et al was also confirmed by strict structure generation (no NSCs) from the 2D NMR data ${ }^{56}$ with the following results: $k=4083 \rightarrow 3874 \rightarrow 1439, t_{g}=12 \mathrm{~m} 6 \mathrm{~s}$. The first ranked structure coincided with structure 14.

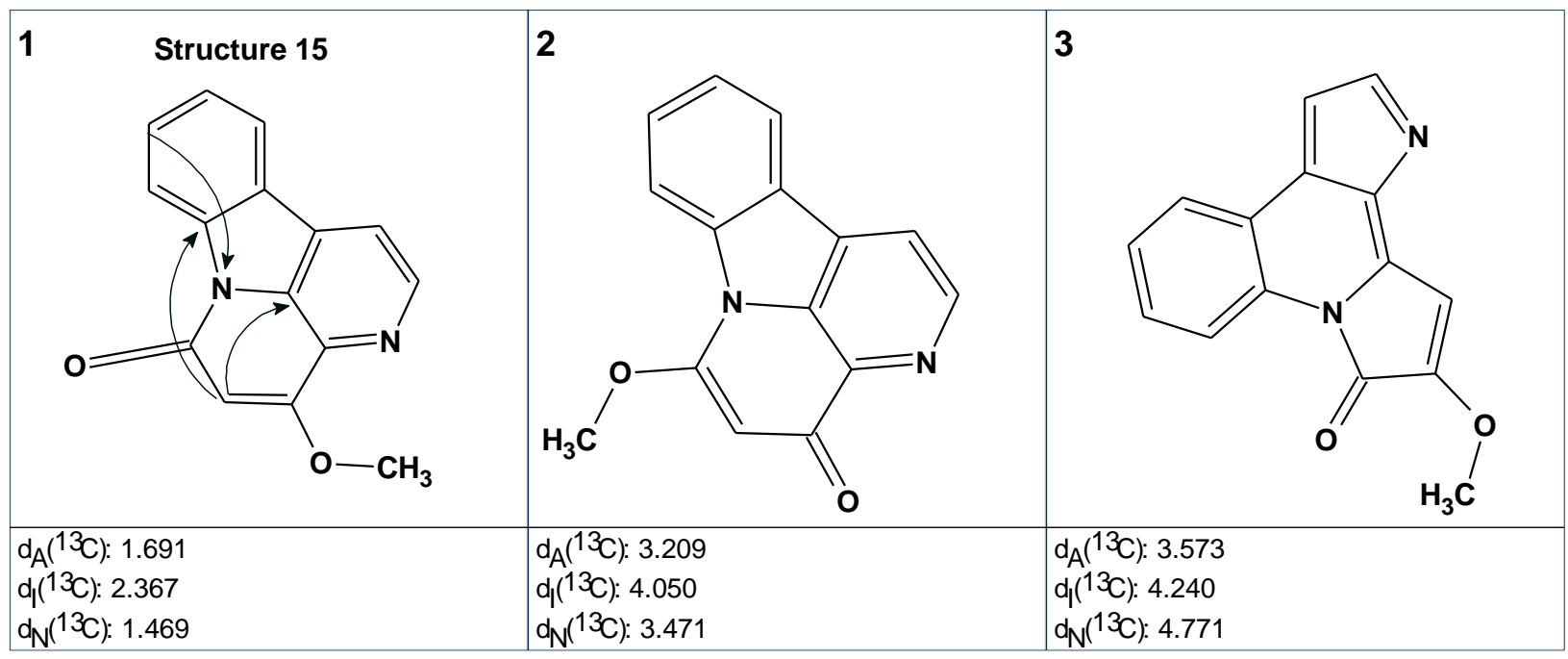

Figure 15. The top ranked structures inferred by the StrucEluc system from the spectral data obtained by Hsieh et $a l^{55}$. The numbers in the top left of each box correspond to the rank ordered structures.

The structure of cordatanine (15) was ranked first by the system. Nonstandard HMBC correlations are shown using arrows. For clarity the differences between the original and revised structures are shown in Figure 16.
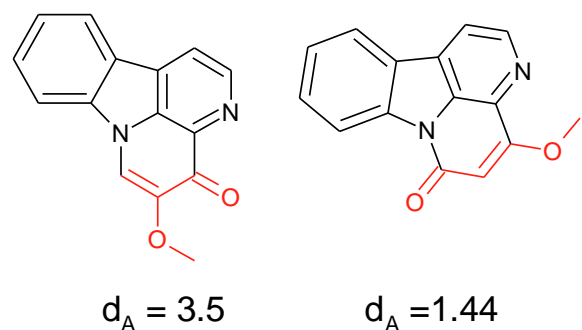

$$
\mathrm{d}_{\mathrm{A}}=3.5
$$$$
d_{A}=1.44
$$

Figure 16. The original and revised structures of drymarietin 
Wetzel et al comment in the conclusion of their article that their results "demonstrate that structure elucidations based only on spectroscopic data bear some risks of misinterpretation" and that "efforts regarding the total synthesis of alkaloids (performed sine ira et studio) helped to identify an erroneous structure assignment". We agree with the authors but our results show that when a software program such as the StrucEluc system is utilized the risks of misinterpretation can be minimized and laborious total synthesis can theoretically be avoided. This example also convincingly shows that ${ }^{13} \mathrm{C}$ chemical shift calculation and dereplication of any isolated natural product are very useful as the first steps towards structure identification. Spectrum prediction frequently allows researcher to recognize if the suggested structure is reliable while dereplication can help to identify the unknown if its structure is already present in a database.

In $2006 \mathrm{Wu}$ et al ${ }^{57}$ isolated a new series of alkaloids, particularly cephalandole A, 16. Using 2D NMR data (not tabulated in the article) they performed a full ${ }^{13} \mathrm{C}$ NMR chemical shift assignment as shown on structure $\mathbf{1 6}$.

Mason et $a l^{58}$ synthesized compound $\mathbf{1 6}$ and after inspection of the associated ${ }^{1} \mathrm{H}$ and ${ }^{13} \mathrm{C}$ NMR data concluded that the original structure assigned to cephalandole $A$ was incorrect. The synthetic compound displayed significantly different data from those given by $\mathrm{Wu}$ et al. The ${ }^{13} \mathrm{C}$ chemical shifts of the synthetic compound are shown on structure 16A.

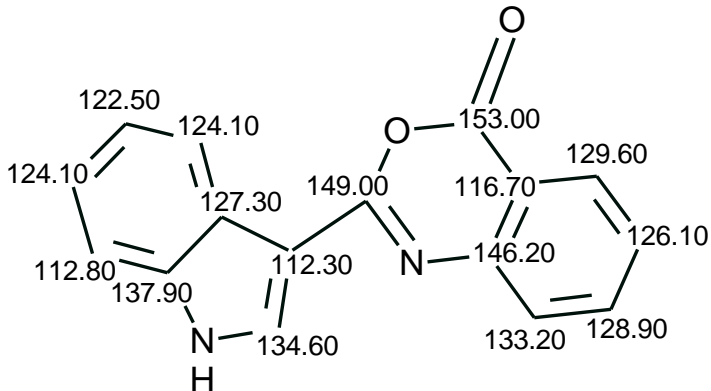

16

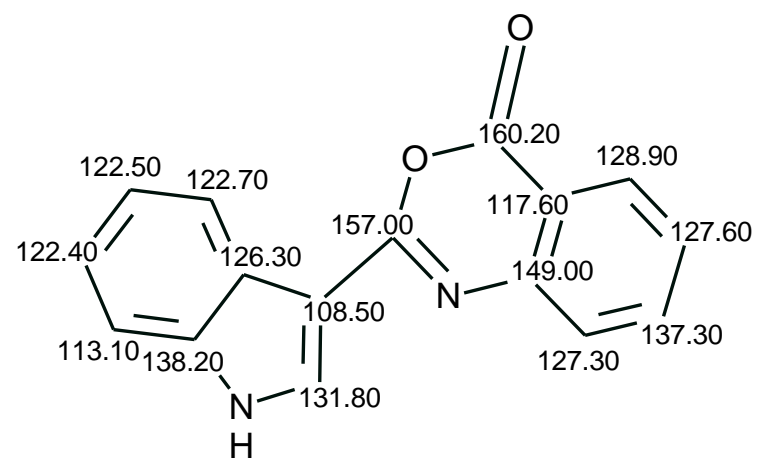

16A

Cephalandole $A$ was clearly a closely related structure with the same elemental composition as 16, and structure $\mathbf{1 7}$ was hypothesized as the most likely candidate. Compound 17 was described in the mid 1960s and this structure was synthesized by Mason et al. The spectral data of the reaction product fully coincided with those reported by $\mathrm{Wu}$ et al. The true chemical shift assignment is shown in structure $\mathbf{1 7}$. 


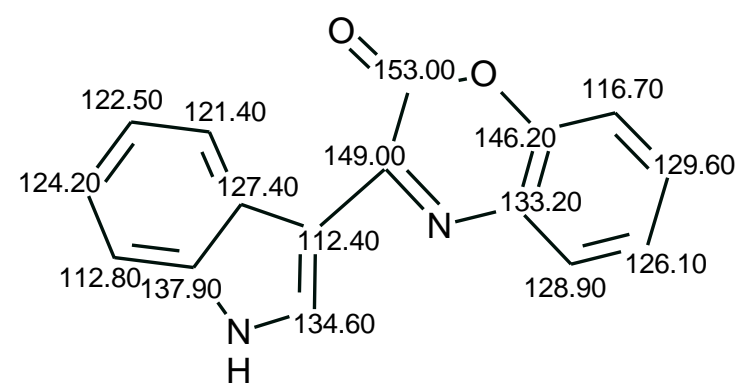

17

For clarity the differences between the original and revised structures are shown in Figure 17.

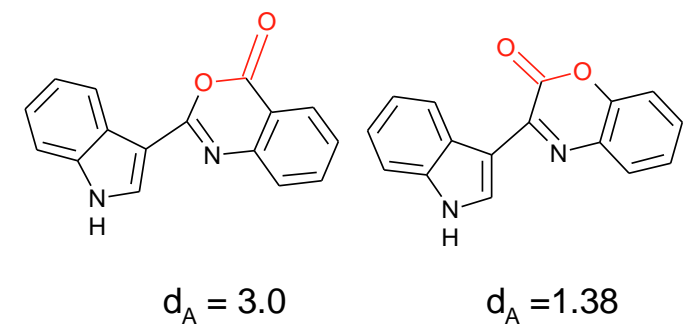

Figure 17. The original and revised structures of cephalandole $A$

We expect that ${ }^{13} \mathrm{C}$ chemical shift prediction, if originally performed for structure $\mathbf{1 6}$, would encourage caution by the researchers (we found $d_{A}=3.02 \mathrm{ppm}$ ). Figure 18 presents the correlation plots of the ${ }^{13} \mathrm{C}$ chemical shift values predicted for structure $\mathbf{1 6}$ by both the HOSE and NN methods versus experimental shift values obtained by Wu et al. The large point scattering, the regression equation, the low $\mathrm{R}^{2}=0.932$ value (an acceptable value is usually $\mathrm{R} 2 \geq 0.995$ ) and the significant magnitude of the $\gamma$-angle between the correlation plot and the 45-degree line (a visual indication for disagreement between the experiment and model) could indicate inconsistencies with the proposed structure and should encourage close consideration of the structure. Our experience has demonstrated that a combination of warning attributes can serve to detect questionable structures even in those cases when the StrucEluc system is not used for structure elucidation. 


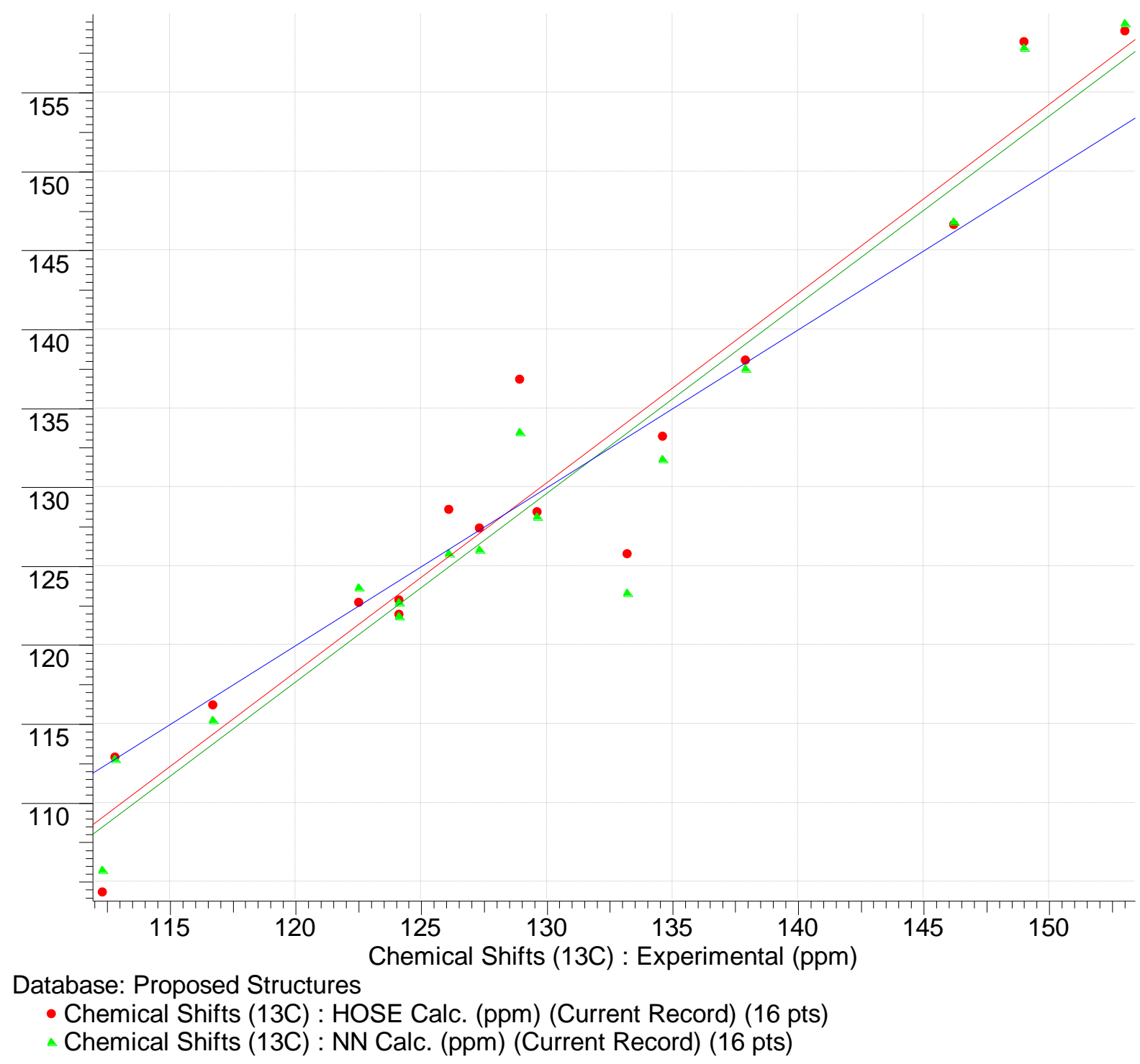

Figure 18. Correlation plots of the ${ }^{13} \mathrm{C}$ chemical shift values predicted for structure $\mathbf{1 6}$ by HOSE and NN methods versus experimental shift values obtained by Wu et al. Extracted statistical parameters: $\mathrm{R}^{2}(\mathrm{HOSE})=0.932, \delta_{\mathrm{HOSE}}=1.20_{\text {हexp }}-25.6$.

In 1988 Sharma et al ${ }^{59}$ isolated two natural products sclerophytins A and B (structures 18 and 19 correspondingly):<smiles></smiles>

18<smiles>C=C1CC[C@H](C(C)C)C2C1[C@H]1O[C@@H]2C[P@]2(C)O[C@@H]1CC[C@@]2(C)OC(C)=O</smiles>

19 
The novel structural features of these oxygen-bridged heterocycles and the significant cytotoxic properties of $\mathbf{1 8}$ have attracted the attention of chemists. At the same time the relative stereochemistry at C-2, C-3, C-6 and C-7 were dubious, and a series of syntheses were undertaken to verify these structures ${ }^{60}$. In consideration of the fact that the synthetic analogs of 18 differed significantly from the originally isolated marine metabolites, an extensive NMR analysis of sclerophytins $A$ and $B$ was undertaken ${ }^{61,62}$. The real structures of these natural products were revealed to be 18A and 19B which are characterized by molecular weights and molecular formulae differing from those found by Sharma et al.

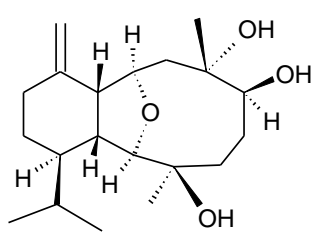

18A

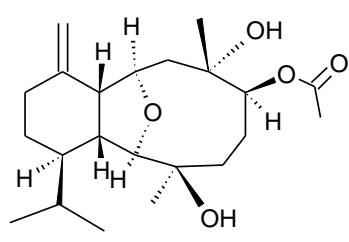

19B

Since the MS and tabulated 2D NMR data of the original structure 18 were not available to us, we carried out ${ }^{13} \mathrm{C}$ chemical shift predictions for structures 18 and $\mathbf{1 8 A}$. The following deviations were obtained:

Structure 18, $\mathrm{d}_{\mathrm{A}}=3.01, \mathrm{~d}_{\mathrm{N}}=2.52, \mathrm{~d}_{\mathrm{A}}(\max )=9.57, \mathrm{R}^{2}(\mathrm{HOSE})=0.985$

Structure 18A, $\mathrm{d}_{\mathrm{A}}=1.37, \mathrm{~d}_{\mathrm{N}}=1,89, \mathrm{~d}_{\mathrm{A}}(\max )=4.95, \mathrm{R}^{2}(\mathrm{HOSE})=0.996$

The data can be used to reject structure 18. The superiority of structure $18 \mathrm{~A}$ is convincingly confirmed by comparison of both deviations and $\mathrm{R}^{2}$ values calculated for structures $\mathbf{1 8}$ and 18a. For clarity the differences between the original and revised structures are shown in Figure 19

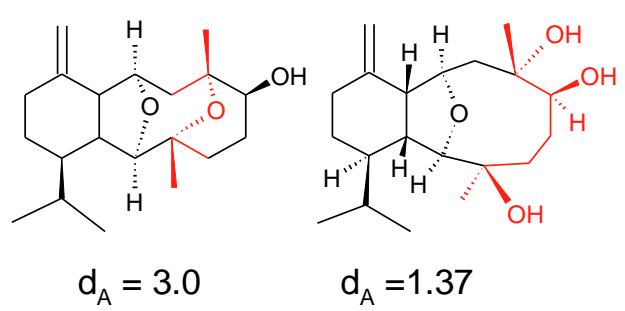

Figure 19. The original and revised structures of sclerophytin A

For revision of the structure of sclerophytin $B$, Friederich et al ${ }^{61}$ synthesized the compound and determined the structure of the reaction product using a combination of mass spectrometry and 2D NMR. When the 1D, HMQC and HMBC data published by the authors ${ }^{61}$ were input into StrucEluc, the system automatically detected the presence of two NSCs in the HMBC data and generated a unique structure, 19B, in $0.17 \mathrm{~s}$ with $d_{A}=1.59$ 
ppm. The solution obtained is evidence that structure 19 is incorrect and could not have been inferred as a candidate from the MS and NMR data presented in the work $^{61}$.

Sakano et al ${ }^{63}$ reported the isolation of the novel lanosterol synthase inhibitors epohelmins A (20) and B (21). The structures were determined by detailed spectroscopic analysis and proposed to be novel 9-oxa-4-azabicyclo[6.1.0]-nonanes. These structure assignments gave rise to doubts based on both chemical and spectroscopic grounds ${ }^{64}$.

Snider and $\mathrm{Gao}^{64}$ comprehensively analyzed both the spectral and chemical aspects of the study of epohelmins A and B. They observed that the originally suggested bicyclo[6.1.0]nonane structures could cyclize readily to give pyrrolizidin-1-ol structures and pointed to the observed chemical shifts as being more consistent with the rearranged product. They suggested structures 22 and $\mathbf{2 3}$ correspondingly as being more appropriate hypotheses.

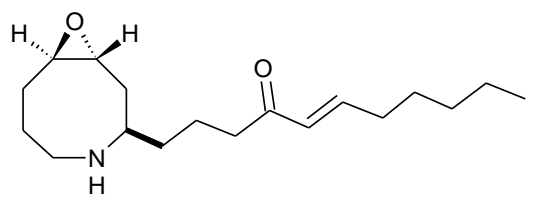

20

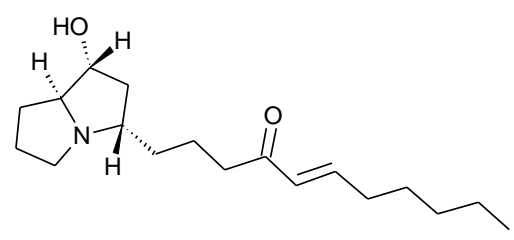

2

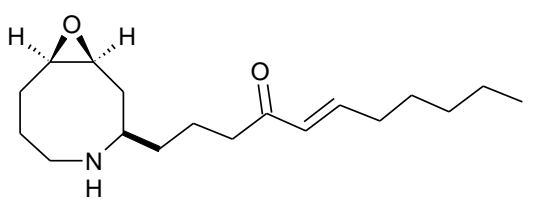

21

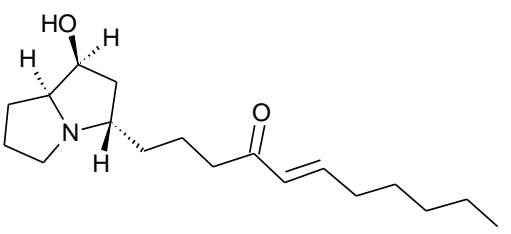

23

To validate their suggestions, the authors ${ }^{64}$ developed an eight step synthesis of epohelmin A (22) and an 11 step synthesis of epohelmin B (23). The ${ }^{1} \mathrm{H}$ and ${ }^{13} \mathrm{C}$ NMR spectra of 22 and 23 were identical to those reported for epohelmin A (20) and epohelmin B (21), and the revised structures of these compounds were therefore unambiguously established via chemical synthesis.

2D NMR spectra of the investigated compounds were not available to us, so only the prediction and comparison of the ${ }^{13} \mathrm{C}$ NMR spectra of competing structures $\mathbf{2 1}$ and $\mathbf{2 3}$ was possible together with review of the discrepancies between the predicted and experimental data (see Table 2). 
Table 2. Comparison of deviations and $\mathrm{R}^{2}$ values calculated for competing structures 21 and 23

\begin{tabular}{|c|c|c|c|c|c|}
\hline Structure & $\mathrm{d}_{\mathrm{A}}, \mathrm{ppm}$ & $\mathrm{d}_{\mathrm{N}, \mathrm{ppm}}$ & $\mathrm{d}_{\mathrm{A}(\max )}$ & $\mathrm{R}^{2}{ }_{(\mathrm{HOSE})}$ & $\mathrm{R}^{2}(\mathrm{~N})$ \\
\hline $\mathbf{2 1}$ & 4.00 & 4.17 & 21.4 & 0.978 & 0.980 \\
\hline $\mathbf{2 3}$ & 1.23 & 1.25 & 4.84 & 0.999 & 0.999 \\
\hline
\end{tabular}

Table 2 unambiguously shows that structure $\mathbf{2 3}$ is superior over structure $\mathbf{2 1}$. For clarity the differences between the original and revised structures are shown in Figure 20.

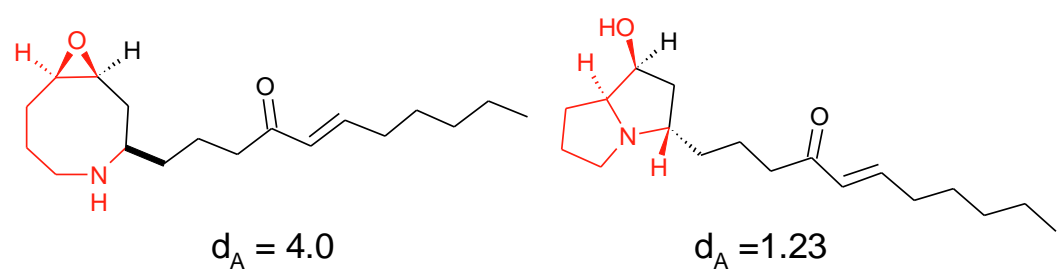

Figure 20. The original and revised structures of epohelmin B

It is likely that if 2D NMR data were available to the researchers then application of StrucEluc would deliver the correct structure very quickly and structure $\mathbf{2 1}$ would immediately be rejected by the program due to the very large deviations, especially with a $\mathrm{d}_{(\max )}$ value of $21.4 \mathrm{ppm}$. Multi-step syntheses would also not be necessary to resolve the structural problem. However, at the same time the method of synthesizing epohelmin A and epohelmin B would not be developed! This contradictory peculiarity of the reassignment problem was strongly underlined in review ${ }^{20}$ where a number of striking examples were given.

In 2000 Hardt et $a l^{65}$ isolated a new cytotoxic marinone derivative neomarinone, molecular formula $\mathrm{C}_{26} \mathrm{H}_{32} \mathrm{O}_{5}$, for which structure 24 was determined from the $1 \mathrm{D}$ and $2 \mathrm{D}$ NMR data:

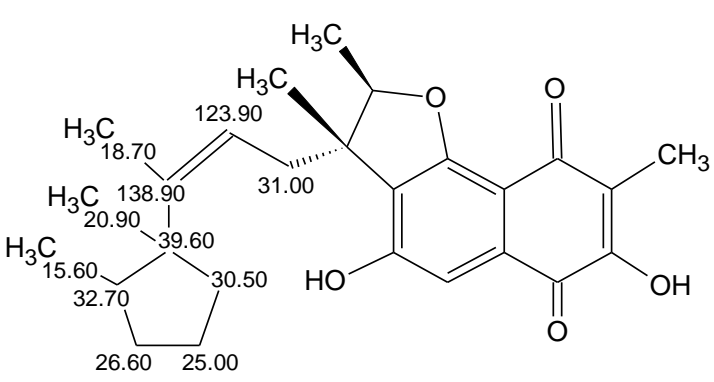

24

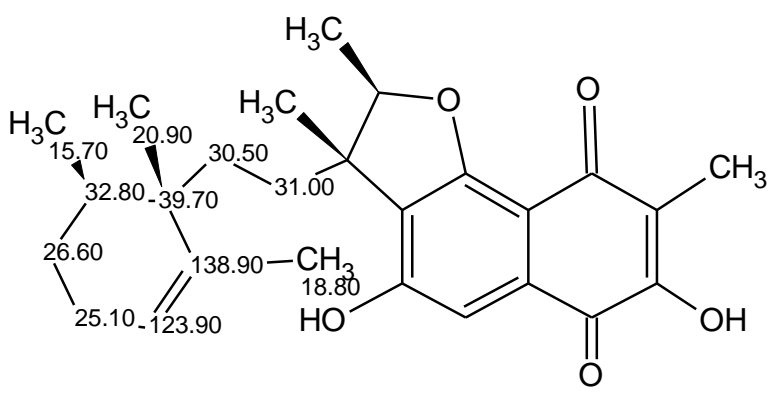

25

The authors noted that the connectivity of the sesquiterpenoid side-chain, and the presence of a methylated cyclopentane ring, were established by ${ }^{1} \mathrm{H}$ NMR, HMBC and COSY data. 
It is worth noting that all $\mathrm{HMBC}$ connectivities between the atoms forming a 5-membered cycle are always of standard length: all combinations of connectivities meet the 2D NMR axioms. This results in difficulties in the unambiguous determination of the atom arrangement in the ring cycle from the HMBC data. The chemical shift assignment for the mentioned fragments is displayed on structure $\mathbf{2 4}$.

On the basis of the novel structure of the sesquiterpenoid unit in neomarinone, in 2003 Kalaitzis et $a l^{66}$ attempted to investigate its biosynthesis via labeling studies with ${ }^{13} \mathrm{C}$ labeled intermediates. The feeding experiments unexpectedly resulted in the modification of the earlier published structure $\mathbf{2 4}$ of neomarinone. The labeling studies and 2D NMR data, including an INADEQUATE experiment, allowed the researcher to obtain evidence that the true structure of neomarinone is $\mathbf{2 5}$. The crucial observation disproving structure 24 was the INADEQUATE connectivity between carbons resonating at 25.10 and 123.90 ppm.

Tabulated 2D NMR data were not available from the original references ${ }^{65,66}$, and it was not possible to apply StrucEluc to this problem. Instead ${ }^{13} \mathrm{C}$ NMR chemical shift prediction was applied to structures $\mathbf{2 4}$ and $\mathbf{2 5}$. The results obtained were:

Structure 24: $\quad \mathrm{d}_{\mathrm{A}}=3.22, \mathrm{~d}_{\mathrm{N}}=3.43, \mathrm{R}^{2}{ }_{\mathrm{HOSE}}=0.995, \mathrm{~d}_{\mathrm{A}(\max )}=9.0$

Structure 25: $\mathrm{d}_{\mathrm{A}}=1.08, \mathrm{~d}_{\mathrm{N}}=2.01, \mathrm{R}^{2}{ }_{\mathrm{HOSE}}=0.999, \mathrm{~d}_{\mathrm{A}(\max )}=5.20$

For clarity the differences between the original and revised structures are shown in Figure 21

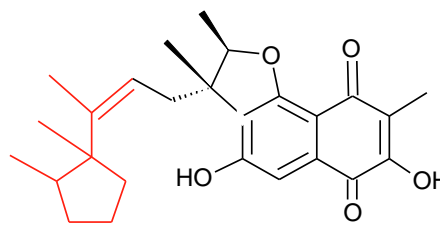

$d_{A}=3.22$

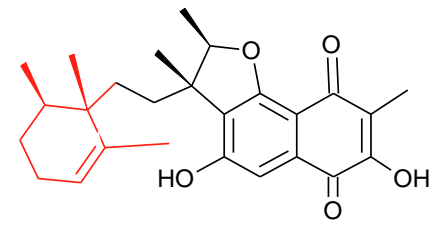

$\mathrm{d}_{\mathrm{A}}=1.08$

Figure 21. The original and revised structures of neomarinone

It is likely that the application of StrucEluc would allow the correct structure to be recognized by its small deviation values in the ranked output file.

\subsection{Revision of structures by the reexamination of 2D NMR data}

In 1992 Suemitsu and coworkers ${ }^{67}$ isolated a new natural product, porritoxin, with molecular formula $\mathrm{C}_{17} \mathrm{H}_{23} \mathrm{NO}_{4}$, for which the following structure was determined from the NMR data: 


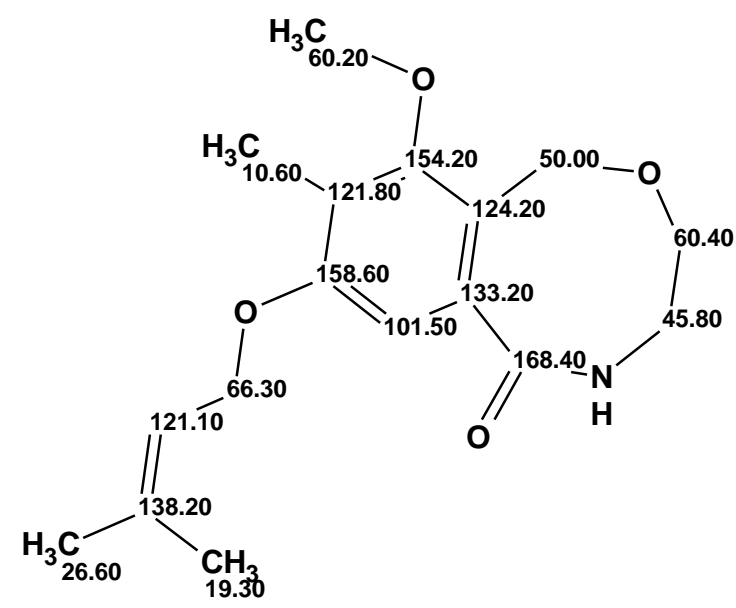

26

In 2002 the same group ${ }^{68}$ reinvestigated the structure of porritoxin by detailed analysis of 2D NMR data including COSY, ${ }^{1} \mathrm{H}-{ }^{13} \mathrm{C}$ HMBC and ${ }^{1} \mathrm{H}-{ }^{15} \mathrm{~N}$ HMBC experiments. This led to the revised structure 27:

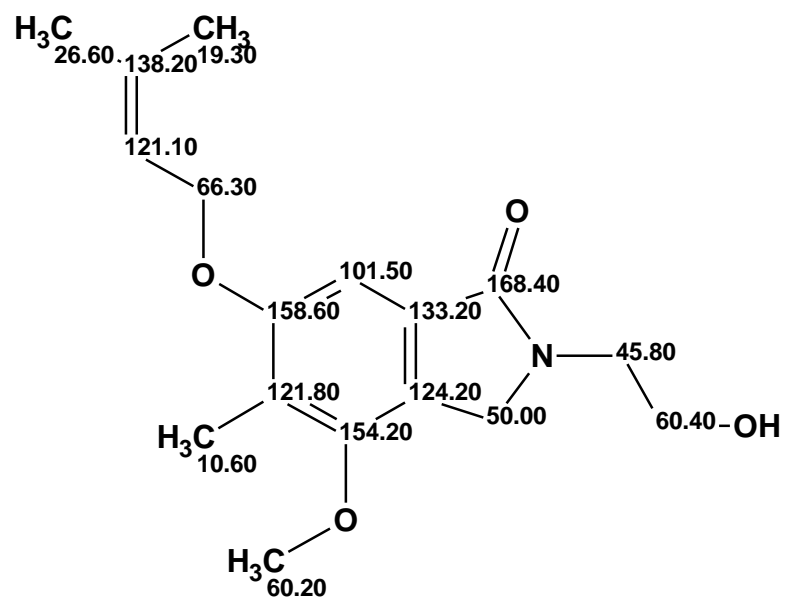

27

Only the ${ }^{1} \mathrm{H}^{-13} \mathrm{C}$ HMBC data were used with the StrucEluc system to produce two structures in $1 \mathrm{~s}$ (see Figure 22) in Fuzzy Structure Generation (FSG) mode (one NSC was detected). The correct structure was reliably distinguished using ${ }^{13} \mathrm{C}$ chemical shift prediction. The original structure $\mathbf{2 6}$ was not generated because the presence of three NSCs must be permitted to allow its generation. For completeness, FSG was restarted with $m=3, a=x$ option ( $m$ is the number of NSCs and $a=x$ means that the lengths of the NSCs are unknown). Results: $\mathrm{k}=52,998 \rightarrow 20,163 \rightarrow 12,573, t_{g}=6 \mathrm{~m} 50 \mathrm{~s}$. Neural net based ${ }^{13} \mathrm{C}$ chemical shift prediction was performed for the output file (calculations took $50 \mathrm{~s}$ ). The correct structure was ranked in first place based on deviations while the original structure was placed only in 59th position with $d_{A}=3.71 \mathrm{ppm}$. The suggested structure for 26 would be immediately rejected if ${ }^{13} \mathrm{C}$ spectrum prediction was performed to check the reliability of the structure assignment. 


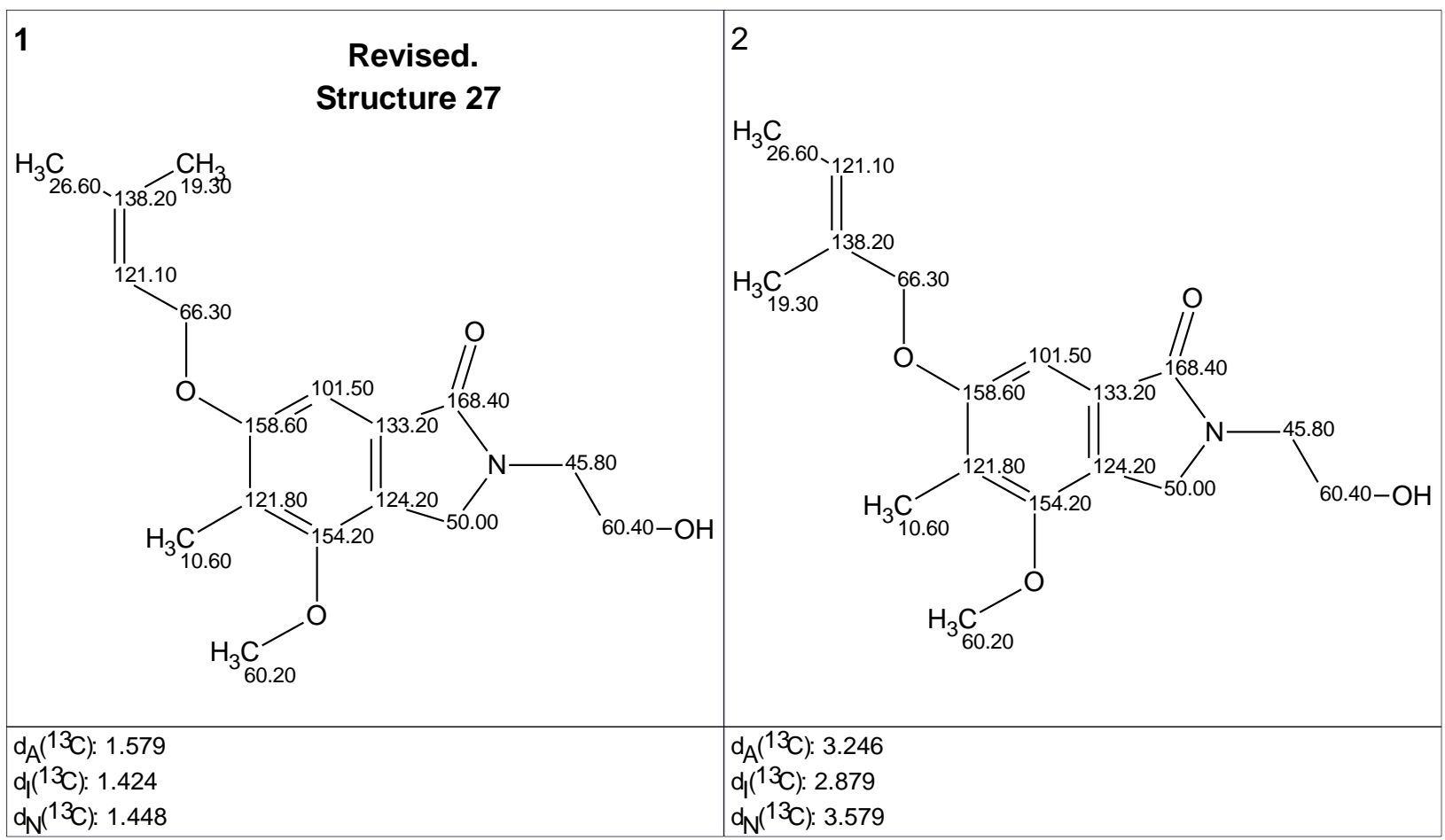

Figure 22. The structures of the output file generated by StrucEluc software for the $\mathrm{C}_{17} \mathrm{H}_{23} \mathrm{NO}_{4}$ compound (porritoxin). The numbers in the top left of each box correspond to the rank ordered structures.

For clarity the differences between the original and revised structures are shown in Figure 23.

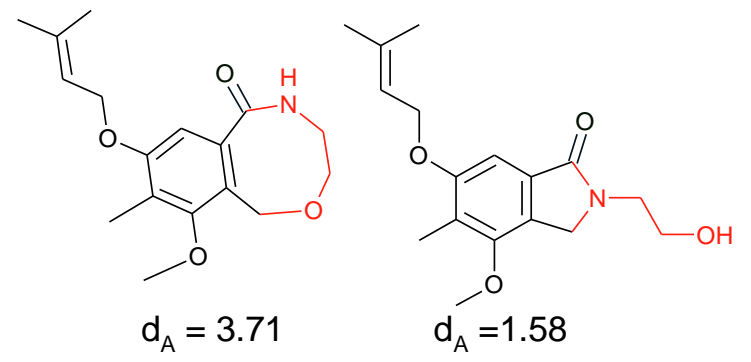

Figure 23. The original and revised structures of porritoxin

Komoda et $a l^{69}$ isolated a new lipoxygenase inhibitor tetrapetalone $A$ (20 mg of material), structure 28, with a molecular formula of $\mathrm{C}_{26} \mathrm{H}_{33} \mathrm{O}_{7} \mathrm{~N}$. The chemical structure was determined using a combination of IR, ${ }^{1} \mathrm{H},{ }^{13} \mathrm{C}$ NMR, DEPT spectra and HMQC, ${ }^{1} \mathrm{H}-{ }^{1} \mathrm{H}$ COSY, HMBC and 2D-INADEQUATE data and by methylation with diazomethane. The authors ${ }^{69}$ inferred structure 28 using a common approach for organic chemists: four fragments were constructed on the basis of the 2D NMR correlations and then the fragments were joined taking into account the HMBC data. The set of mentioned fragments that should be present in the analyzed structure can be 
considered as a set of structural axioms. The stereochemistry was investigated by the coupling constants in ${ }^{1} \mathrm{H}$ NMR, NOESY data and the modified Mosher's method ${ }^{70}$.

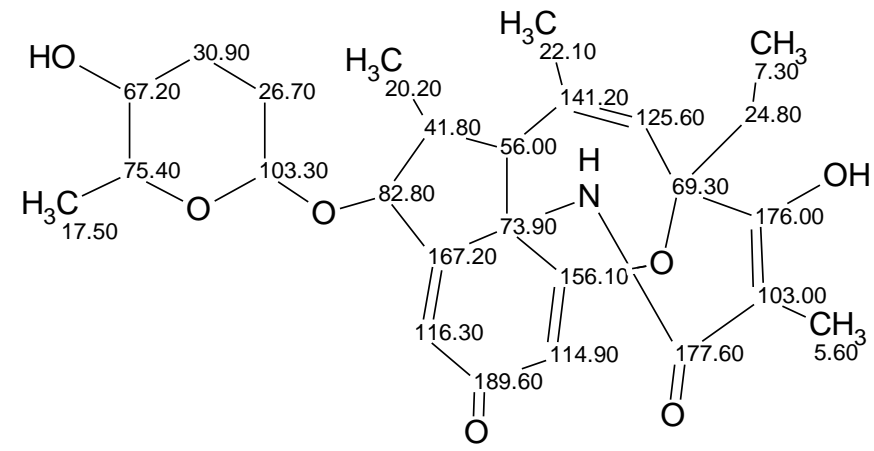

28

All available spectral data and the associated postulated fragments were input into StrucEluc. The fragments were drawn into the molecular connectivity diagram window ${ }^{25,26}$, MCD, as shown in Figure 24. The chemical bonds are denoted by black lines and the HMBC correlations by green lines.

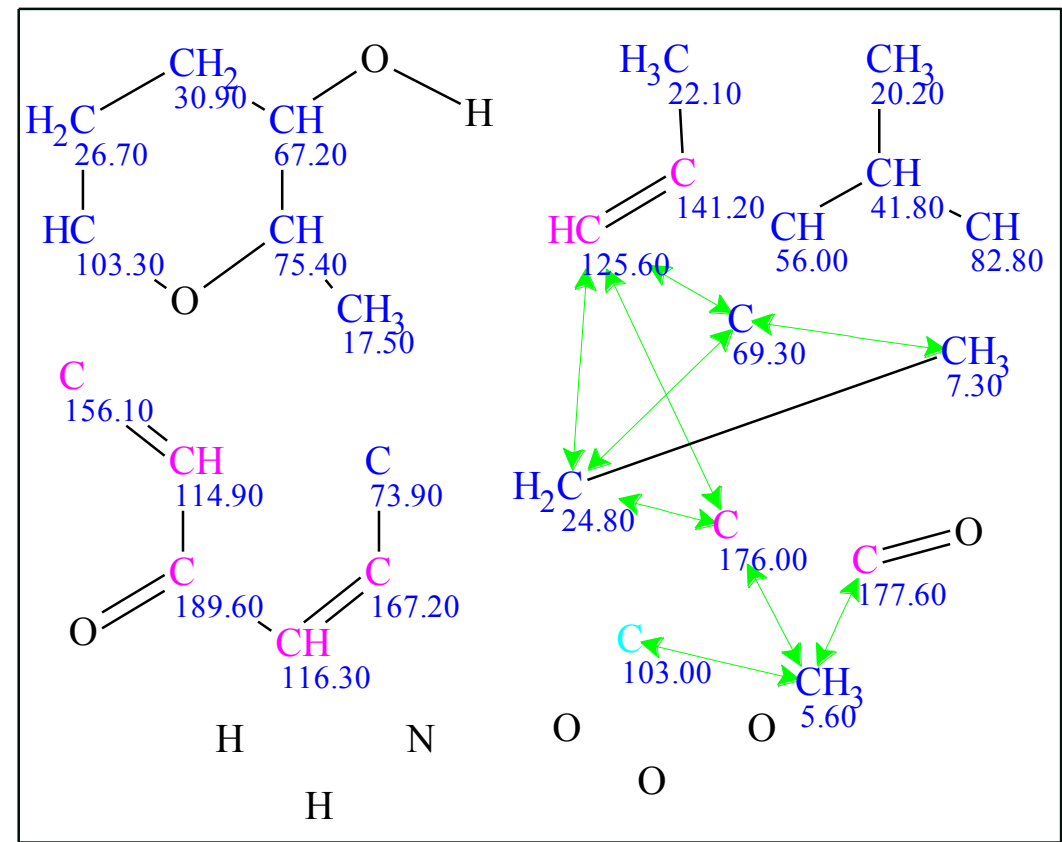

Figure 24. The molecular connectivity diagram (MCD) which shows the fragments suggested by the authors ${ }^{69}$ and used by the StrucEluc software for the purpose of structure generation. The green arrows denote the HMBC correlations and the black lines the chemical bonds. The following colors are used to denote the atom hybridizations: $s p^{2}-$ violet, $s p^{3}-$ blue, not $s p-$ sky blue.

Structure generation from the MCD led to the following results: $k=16,465 \rightarrow 13,672 \rightarrow 9,203$ and $t_{g}=61 \mathrm{~s}$. Ranking the output file in ascending order of mean average error values placed structure 28 into 111th position. The first two structures and the structure occupying position 111 are shown in Figure 25. 


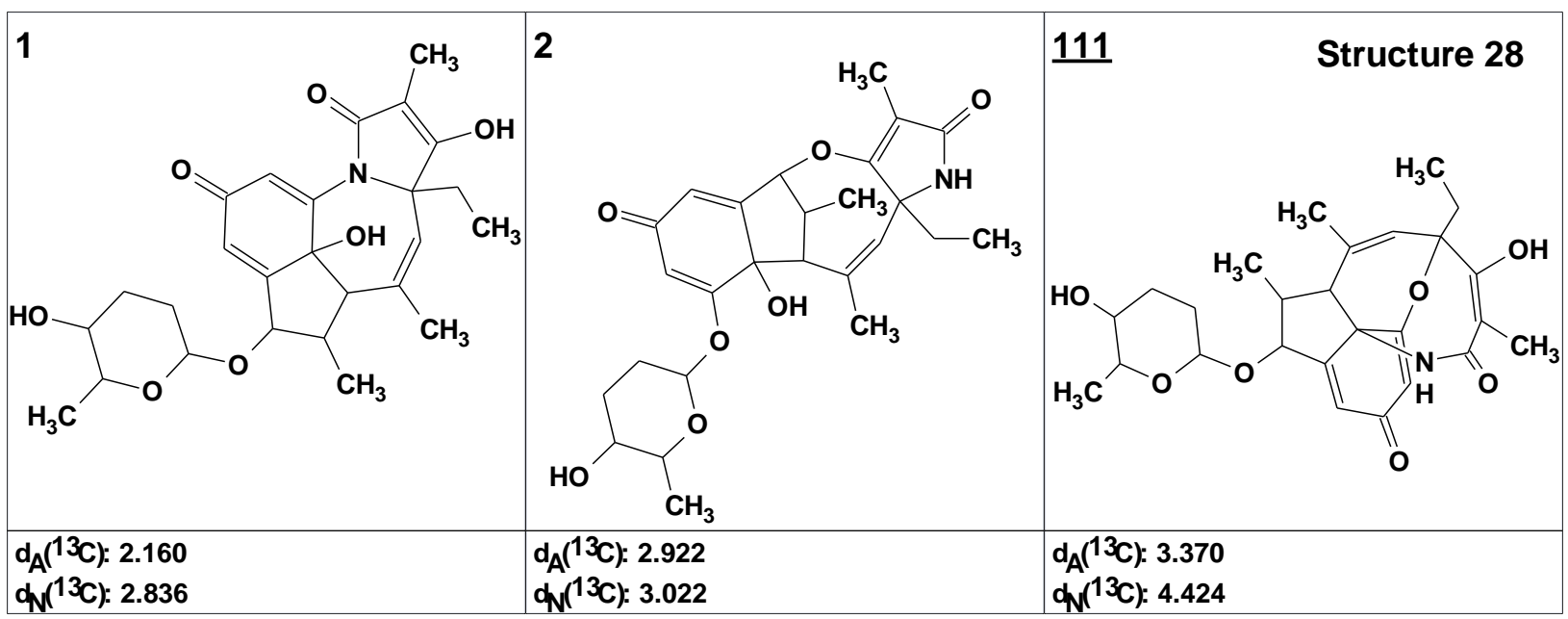

Figure 25. The first, second and 111th structures in the ranked output file produced by StrucEluc as a solution to the problem of tetrapetalone $A$ structure elucidation. The 111th structure is equivalent to structure $\mathbf{2 8}$ of tetrapetalone $A$ suggested by other authors. ${ }^{69}$ The numbers in the top left of each box correspond to the rank ordered structures.

The automatically obtained solution to the problem delivered the best structure from among almost 10,000 candidates. The structure was characterized by deviation values that were significantly smaller than those found for structure $\mathbf{2 8}$. It should be obvious that structure $\mathbf{2 8}$ cannot be the correct structure.

The same group ${ }^{71}$ undertook a reinvestigation of tetrapetalone $A$ structure. In this study the ${ }^{1} \mathrm{H}-{ }^{15} \mathrm{~N}$ HMBC data were used to provide more convincing evidence of the structural conclusions. As a result structure $\mathbf{2 8}$ was revised and the following structure was assigned to tetrapetalone $A$. The stereochemistry was determined as shown below:

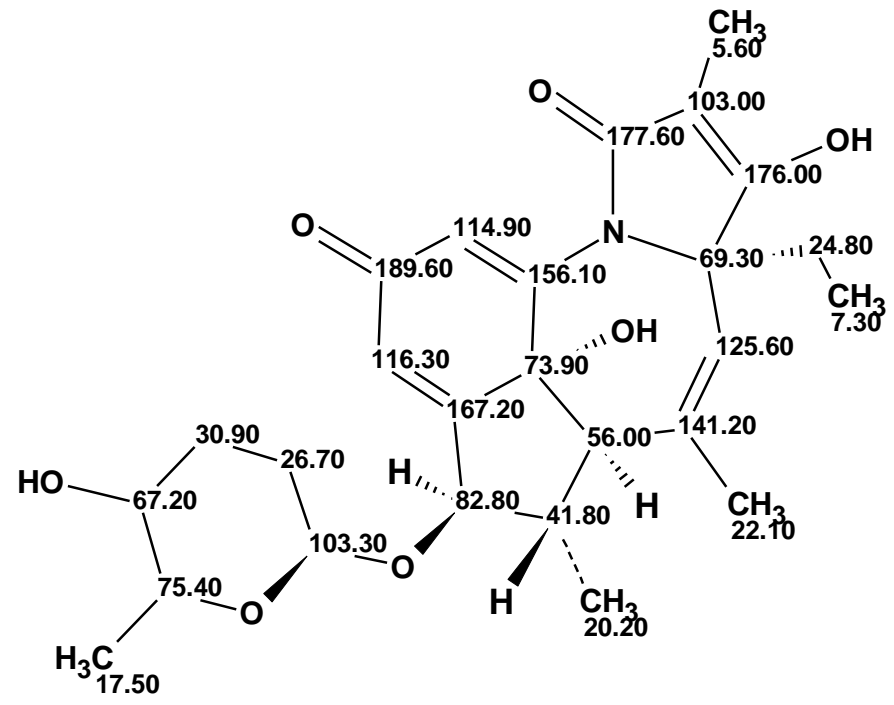

29

Comparison of structure $\mathbf{2 9}$ with the first structure in Figure 25 leads to conclusion that the StrucEluc system has generated and automatically selected the true structure of tetrapetalone $A$ without using any additional information. The structure could therefore be 
correctly identified in several minutes if the StrucEluc system was used for solving this problem. Moreover, all 256 stereoisomers of structure 29 were generated and HOSE-code based ${ }^{13} \mathrm{C}$ chemical shift calculation was performed to select the most probable stereochemistry which also coincided with the stereoconfiguration shown in structure 29. For clarity the differences between the original and revised structures are shown in Figure 26.

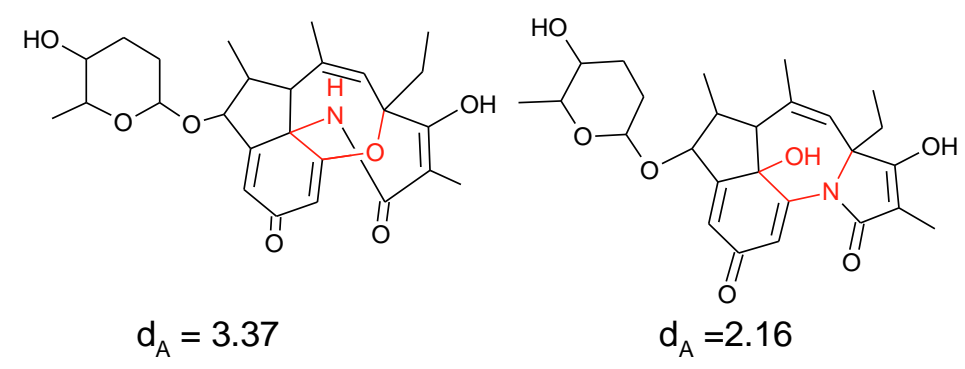

Figure 26. The original and revised structures of tetrapetalone $A$

In 1990 Cáceres et $\mathrm{l}^{72}$ isolated the dolabellane diterpenoid palominol of molecular formula $\mathrm{C}_{20} \mathrm{H}_{32} \mathrm{O}$, for which structure 30 was suggested with the shown ${ }^{13} \mathrm{C}$ shift assignment:

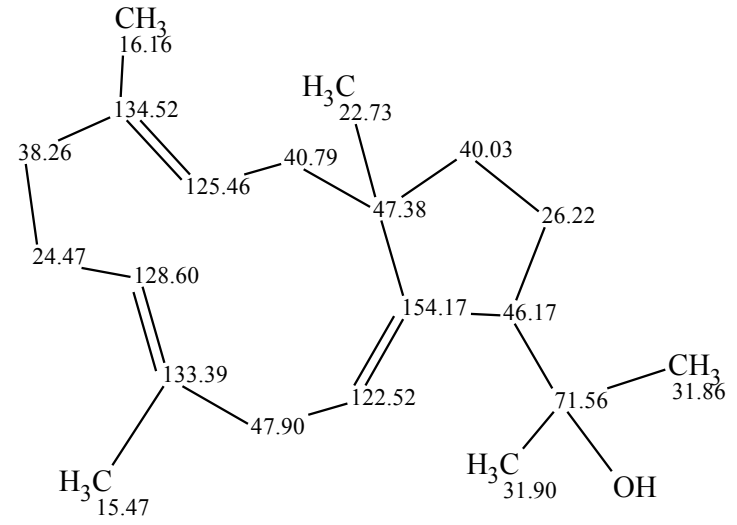

30

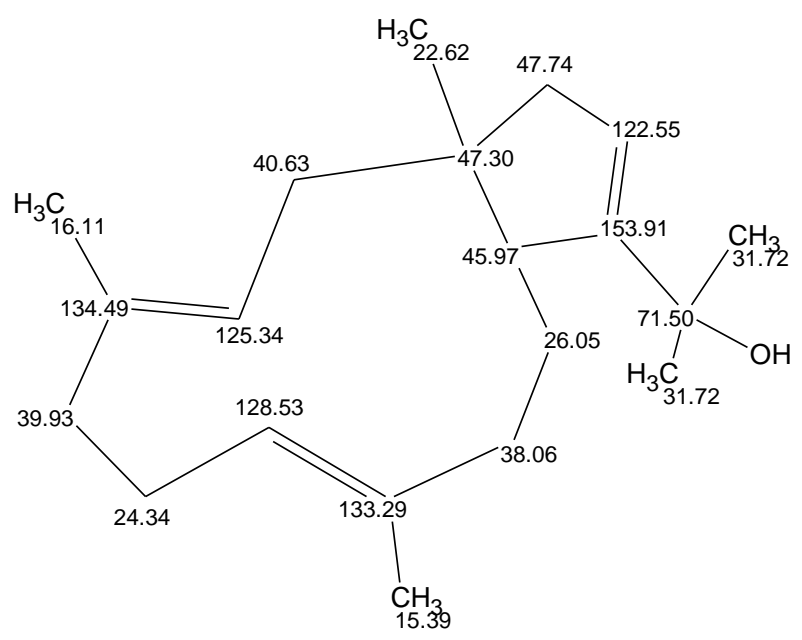

31

In 1993 the same group ${ }^{73}$ reinvestigated structure 30 using HMQC, HMBC, COSY, INADEQUATE and ROESY data and established that structure $\mathbf{3 1}$ was the actual structure. Using the StrucEluc system and utilizing 1D NMR, HMQC and HMBC data we obtained four structures in $1 \mathrm{~s}$ in Fuzzy Generation Mode with one NSC detected by the program. Structure 30 was not generated at all. Our studies showed that many NSCs, around 8, would need to be present in the HMBC data to allow it to be generated. ${ }^{13} \mathrm{C}$ chemical shift prediction was performed for the four candidate structures. In so doing both the cis- and trans- configurations of the double bonds included into the 11-membered ring were taken into account. The smallest deviations $\left(d_{A}=2.18 \mathrm{ppm}\right)$ were found for the trans- 
configurations and the priority of structure 31 was confirmed (for double bond transconfigurations in structure 30 , the value $d_{A}=2.56 \mathrm{ppm}$ was found). For clarity the differences between the original and revised structures are shown in Figure 27.
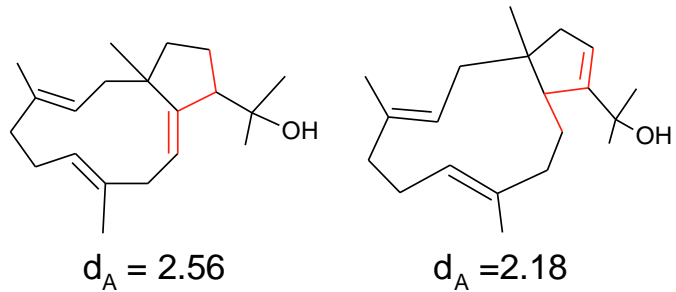

Figure 27. The original and revised structures of palominol

Further testing of the StrucEluc system used the experimental data of Krishnaiah et $a l^{74}$ for the structure elucidation of a newly separated alkaloid lamellarin $\gamma$. The following structure was deduced by the authors ${ }^{74}$ from the molecular formula $\mathrm{C}_{30} \mathrm{H}_{27} \mathrm{O}_{9} \mathrm{~N},{ }^{1} \mathrm{H},{ }^{13} \mathrm{C}$ NMR spectra and 2D NMR data (HMQC, HMBC and NOESY):

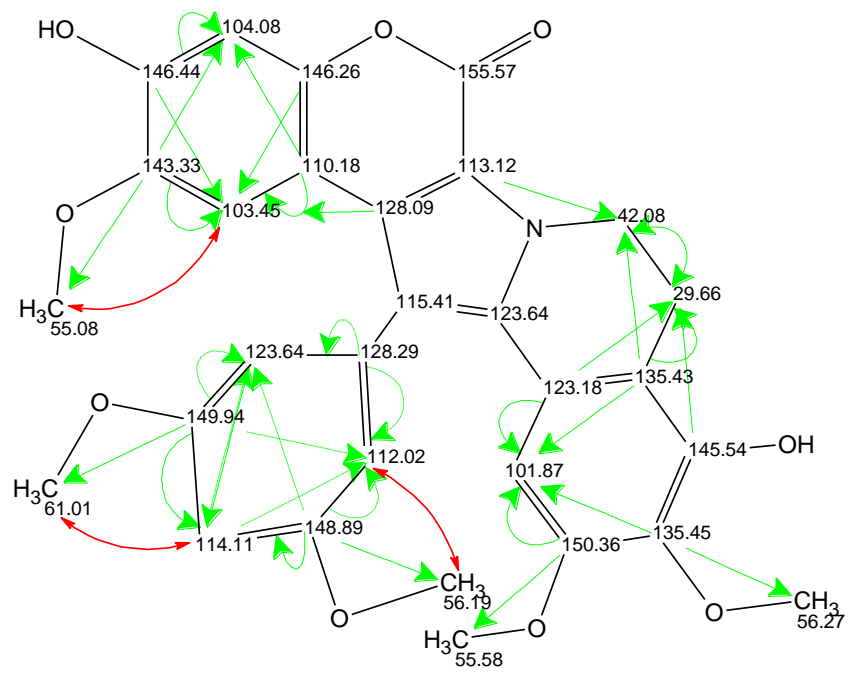

32

The chemical shift assignments suggested in the original work ${ }^{74}$ are shown on the chemical structure 32. The green arrows indicate HMBC correlations, while the double-sided red arrows show the NOESY correlations. The dotted green lines are used to denote ambiguous connectivities. It is obvious that the structure is in agreement with the suggestion that all HMBC correlations are of a standard length (2-3 bonds, ${ }^{2-3} \mathrm{~J}_{\mathrm{CH}}$ ), while the NOESY correlations support the structure only in those cases when the methoxy groups at 61.01 and $56.19 \mathrm{ppm}$ are asymmetrically oriented on the 1,3,5-trisubstituted benzene ring. The chemical shift assignment of structure 32 shows that the chemical shifts of the 1,3,5-trisubstituted benzene ring and the methoxy groups do not meet the local symmetry of this fragment. There is no reason that the theoretically symmetric carbons at 112.0 and 123.6 ppm should be so distinct. 
Considering this observation we $\mathrm{e}^{75}$ performed ${ }^{13} \mathrm{C}$ chemical shift prediction for structure 32 using ACD/NMR Predictors ${ }^{43}$ based on both the HOSE code and neural nets algorithmic approaches. The following results were obtained: $d_{A}=4.70 \mathrm{ppm}, d_{N}=5.29 \mathrm{ppm}$. It is obvious that the calculated deviations are extremely high in terms of providing confirmation of structure 32 . The correlation plots of the ${ }^{13} \mathrm{C}$ chemical shift values predicted for structure 32 via both prediction approaches are presented in Figure 28.

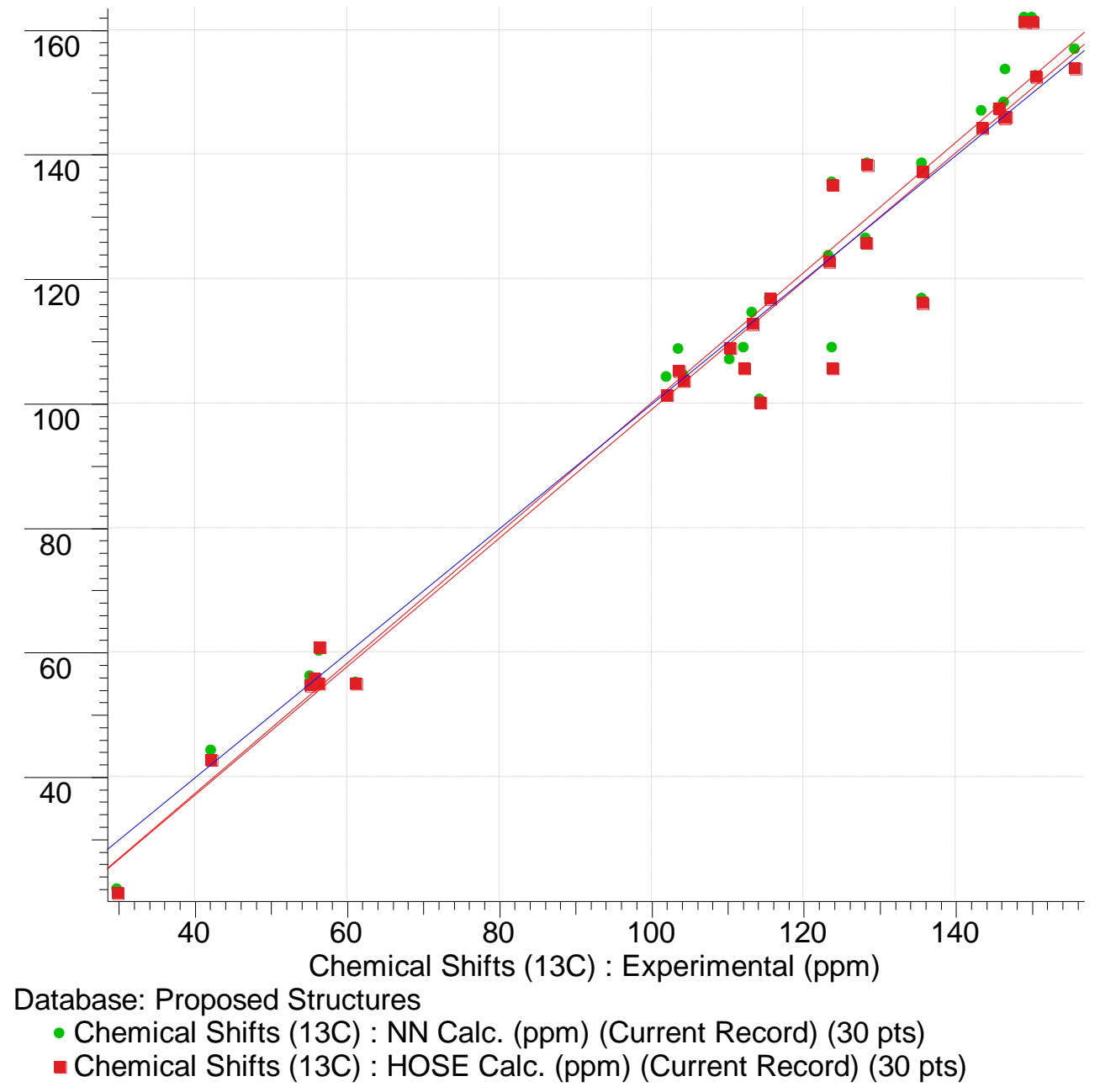

Figure 28. Correlation plots of ${ }^{13} \mathrm{C}$ chemical shift values predicted for structure 32 by HOSE (red points) and $\mathrm{NN}$ (green points) prediction methods versus experimental shift values. The target line $\mathrm{Y}=\mathrm{X}$ is colored in blue. The $\mathrm{R}^{2}$ value calculated by HOSE based method is equal to 0.965 .

The data shown in Figure 28 and represented by the statistical parameters indicates that the calculated ${ }^{13} \mathrm{C}$ NMR chemical shifts differ significantly from the experimental values. This observation encouraged us to apply StrucEluc to validate the assignment. 
The molecular formula and associated spectral data ${ }^{74}$ were input into StrucEluc and a molecular connectivity diagram (MCD) was created. An attempt to perform structure generation in Common Mode $^{26}$ where possible structures are assembled from "free" atoms indicated that solving the problem would be extremely time consuming. This is accounted for by a deficit in the number of hydrogen atoms in the molecular formula where the double bond equivalent $\mathrm{DBE}=18$. A lack of HMBC correlations can be observed in structure 32. According to a general methodology described elsewhere ${ }^{26}$ in such a situation the application of fragments stored into the system Fragment Library can be helpful. A fragment search using ${ }^{13} \mathrm{C}$ NMR chemical shifts resulted in the selection of 2,318 fragments whose ${ }^{13} \mathrm{C}$ chemical shifts agreed with the experimental spectrum. The Found Fragments, ranked in descending order of carbon atom numbers, are displayed in the software program and fragments placed at the top of the ranked file are considered as the most likely since they consume a large number of skeletal atoms. For instance, in the case described here the first fragment had the molecular formula $\mathrm{C}_{17} \mathrm{H}_{10} \mathrm{NO}_{4}$ and the ${ }^{13} \mathrm{C}$ chemical shifts of the fragment were close to those observed experimentally.

The MCD creation procedure was applied to the top ten ranked Found Fragments, and 192 MCDs were produced. Each MCD contained only one fragment - the first ranked one, and the observed difference between the MCDs was in regards to the chemical shift assignments of fragment carbons performed automatically by the software program. Consequently, the lengths of the HMBC correlations corresponding to different pairs of associated chemical shifts in the different MCDs are different. Fuzzy Structure Generation ${ }^{34}$ was initiated with the following options: $m=0-20 ; a=x$ (the augmentations of the connectivities are unknown) and was completed in 11 min with the following results: $k=133504 \rightarrow 120816 \rightarrow 1530$. The chemical shift prediction for ca. 121,000 molecules took $11 \mathrm{~min}$.

Structure 33, characterized by $d_{A}=1.26 \mathrm{ppm}$ and $d_{N}=2.55 \mathrm{ppm}$ was distinguished as the best structure: 


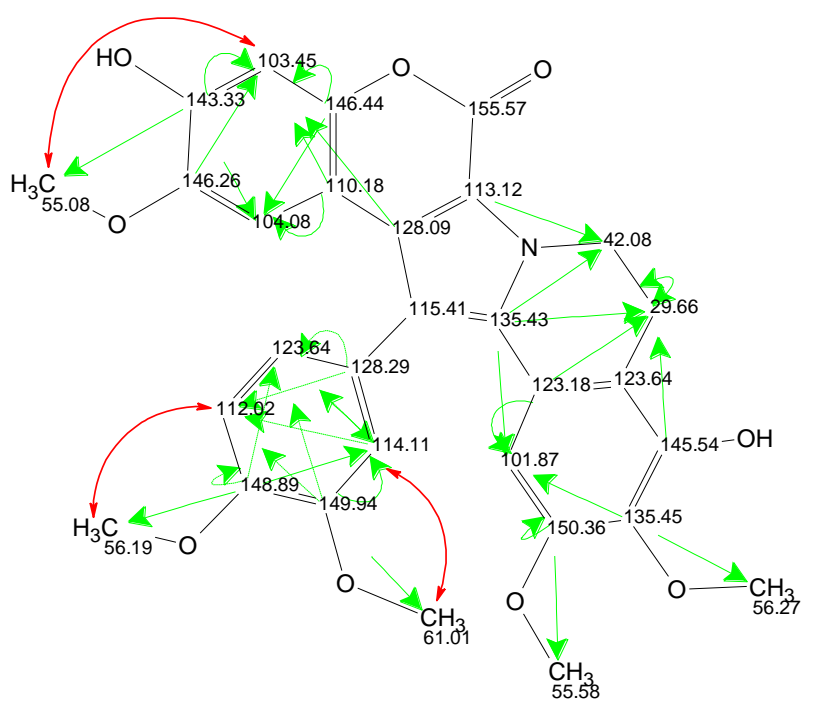

33

A comparison of deviations calculated for structures $\mathbf{3 2}$ and $\mathbf{3 3}$ shows that structure $\mathbf{3 3}$ is much more probable. However structure $\mathbf{3 3}$ possesses an attribute which suggests that there may be a need for chemical shift reassignment: one of the four NOESY correlations (see the left portion of structure 33) does not make sense chemically. At the same time structure 32, suggested by the authors ${ }^{74}$, was also generated by the program and placed in 21 st position by the ranking procedure. This also confirms the superiority of structure $\mathbf{3 3}$ over structure $\mathbf{3 2}$.

The next step was to automatically find the chemical shift assignments of structure $\mathbf{3 3}$ which are in accord with both the HMBC and NOESY correlations. As shown above, there are a lot of identical structures among the $>120 \mathrm{k}$ structures generated from the 192 MCDs. For our purpose, we collected all isomorphic structures for structure 33, 384 in total, in a separate file. We then performed NMR spectral predictions and ranked the file. The structure ranked first fit both the HMBC and NOESY spectra and structure 33A was finally selected.

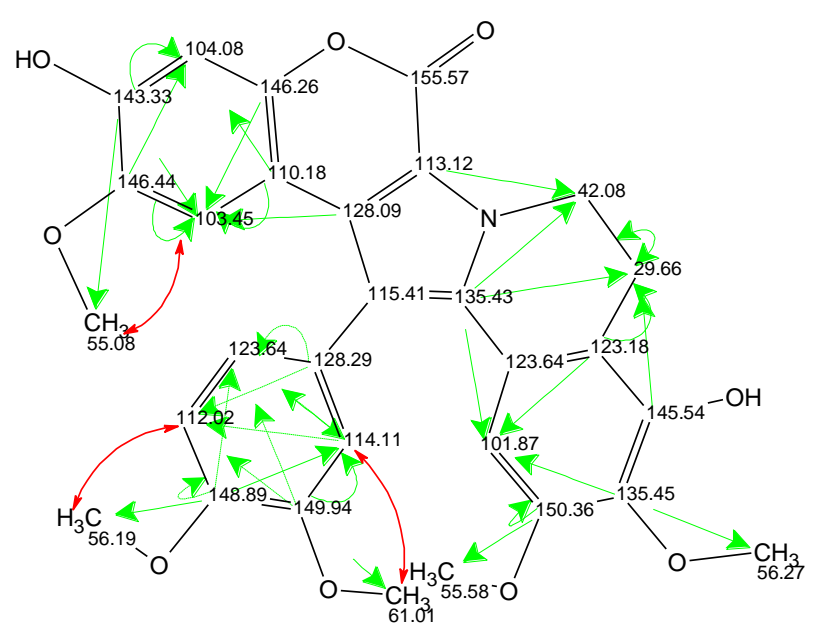

33A 
Deviations and $\mathrm{R}^{2}$ values calculated for structures $\mathbf{3 2}$ and $\mathbf{3 3 A}$ are presented in Table 3.

Table 3. Comparison of deviations and $\mathrm{R}^{2}$ values calculated for competing structures 32 and 33A.

\begin{tabular}{|c|c|c|c|c|c|}
\hline Structure & $d_{A}, \mathrm{ppm}$ & $d_{N}, \mathrm{ppm}$ & $d_{A}(\max )$ & $\mathrm{R}^{2}(\mathrm{HOSE})$ & $\mathrm{R}^{2}(\mathrm{NN})$ \\
\hline 32 & 4.70 & 5.29 & 18 & 0.965 & 0.967 \\
\hline 33A & 1.26 & 2.55 & 5 & 0.997 & 0.993 \\
\hline
\end{tabular}

Table 3 shows the evident superiority of structure 33A over structure 32. For clarity the differences between the original and revised structures are shown in Figure 29.

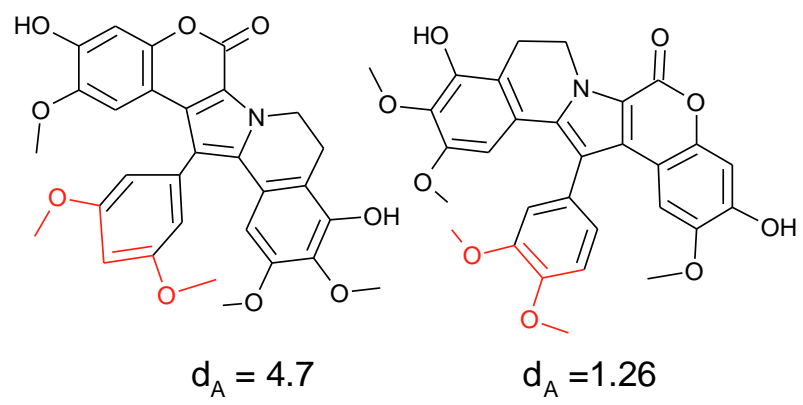

Figure 29. The original and revised structures of lamellarin $\gamma$

In 2004 Hiort et $a l^{76}$ isolated from the Mediterranean sponge Axinella damicornis seven new natural products including four pyranonigrins featuring a novel pyrano[3,2$b$ ]pyrrole skeleton previously unknown in nature. All structures were elucidated on the basis of extensive one- and two-dimensional NMR spectroscopic studies $\left({ }^{1} \mathrm{H},{ }^{13} \mathrm{C}, \mathrm{COSY}\right.$, HMQC, HMBC, NOE difference spectra) and MS analysis. For the two chiral pyranonigrin molecules, particularly for pyranonigrin $A,\left(\mathrm{C}_{9} \mathrm{H}_{10} \mathrm{NO}_{5}, \mathrm{DBE}=7\right) \mathbf{3 4}$, the absolute configurations were established by quantum mechanical calculations of their circular dichroism (CD) spectra.

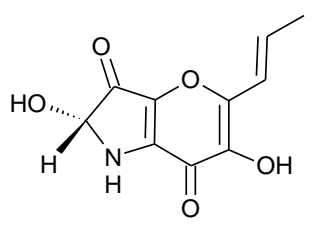

34

In 2007 Schlingmann et $a l^{77}$ isolated from the marine fungus Aspergillus niger a compound of molecular formula $\mathrm{C}_{9} \mathrm{H}_{10} \mathrm{NO}_{5}$ whose physical data were identical to those published by Hiort et $a l^{76}$ for pyranonigrin A. Interpretation of the NMR data did not permit the authors ${ }^{77}$ to assign structure 34 to pyranonigrin A. They suggested that the correct structure is one of the following three possible candidates: 
<smiles>CC=Cc1oc2c(c(=O)c1O)C(O)NC2=O</smiles>

34b<smiles>C/C=C/c1oc2c(c(=O)c1O)C(=O)N[C@H]2O</smiles>

$34 c$

Similar to the previous report ${ }^{76}$ the structure determination of the pyranonigrin A was based on the interpretation of spectroscopic data, especially MS and NMR data, which included HSQC, COSY, ROESY, HMBC, and an essential ${ }^{1} \mathrm{H}-{ }^{15} \mathrm{~N}$ HMBC. Comprehensive analysis of the experimental 1D and 2D NMR spectra allowed authors ${ }^{77}$ to reject hypotheses 34b and 34c. It was concluded that pyranonigrin A was consistent with structure 34d. To further prove this finding the researchers produced hydrophobic derivatives of the analyzed compound suitable for comparison of experimental UV/CD spectra with that of $a b$ initio predicted data (in vacuo), since the substance itself was soluble only in polar solvents. As a result of extensive experimental and theoretical investigations, the structure of pyranonigrin $A$ was unambiguously elucidated, and its absolute configuration was determined.

The initial spectral data presented for pyranonigrin $A$ by Hiort et al were input into the StrucEluc system, and strict structure generation was performed excluding any NSCs as the authors ${ }^{76}$ had suggested (an axiom). The results gave: $k=109 \rightarrow 81 \rightarrow 72, t_{g}=0.3 \mathrm{~s}$. The first and sixth ranked structures are presented in Figure 30.

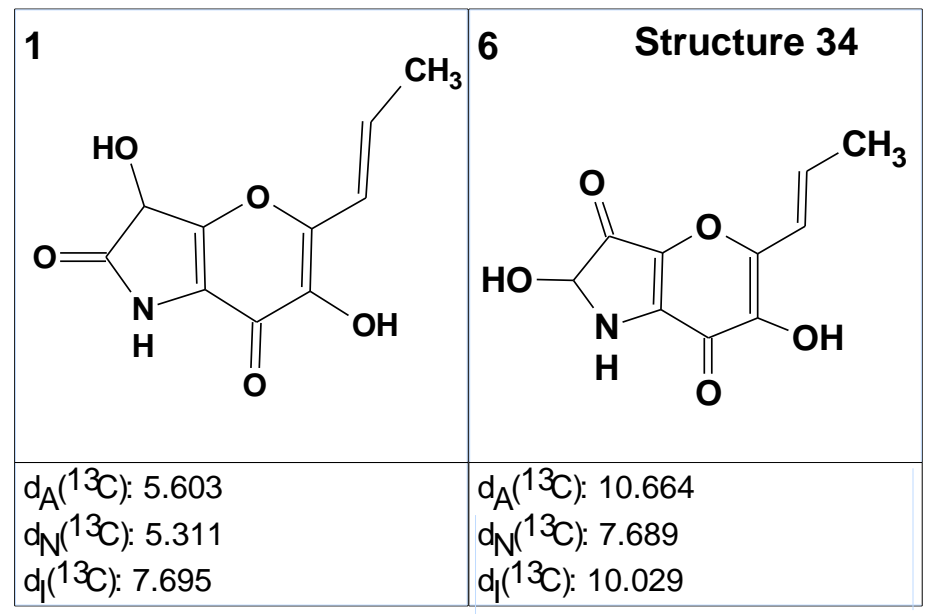

Figure 30. The first and sixth ranked structures of the output file produced using strict structure generation for pyranonigrin $A$. The numbers in the top left of each box correspond to the rank ordered structures. 
The first ranked structure, similar to $\mathbf{3 4}$, is characterized by unacceptably large deviations, while the suggested original structure $\mathbf{3 4}$ should be immediately rejected as it had a large deviation of $d_{A}=10.6 \mathrm{ppm}$. The hypothesized structures $\mathbf{3 4 b}-\mathbf{3 4 d}$ were not generated at all. As mentioned earlier, large deviations found for the first ranked structure should be considered as an indication to the possible presence of non-standard correlations in the 2D NMR data. The next step was Fuzzy Structure Generation with options $m=1, a=$ $x$ to provide the result: $k=3024 \rightarrow 2130 \rightarrow 1144, t_{g}=14 \mathrm{~s}$. The correct structure $\mathbf{3 4 d}$ was generated and ranked first $\left(d_{A}=2.03\right)$, structure $\mathbf{3 4 \mathbf { c }}$ was ranked fifth $\left(d_{A}=5.26\right)$ and structure $\mathbf{3 4}$ was placed in 31 st position. Structure $\mathbf{3 4 b}$ was not generated.

To check the solution for stability we performed fuzzy structure generation using $m=2$ and $a=x$ as options to provide the following results: $k=18275 \rightarrow 10725 \rightarrow 3506, t_{g}=2 \mathrm{~m} 23 \mathrm{~s}$. Under the condition that two NSCs may present in a structure, all structures $(\mathbf{3 4}, \mathbf{3 4 b - 3 4 d})$ considered by the authors ${ }^{77}$ were generated. During this run, the program produced a full set of structures containing all six possible rearrangements of $\mathrm{OH}, \mathrm{NH}$ and $\mathrm{C}=\mathrm{O}$ groups on the 5-membered cycle. These structures along with their rank ordered positions in the output file are presented in Figure 31.

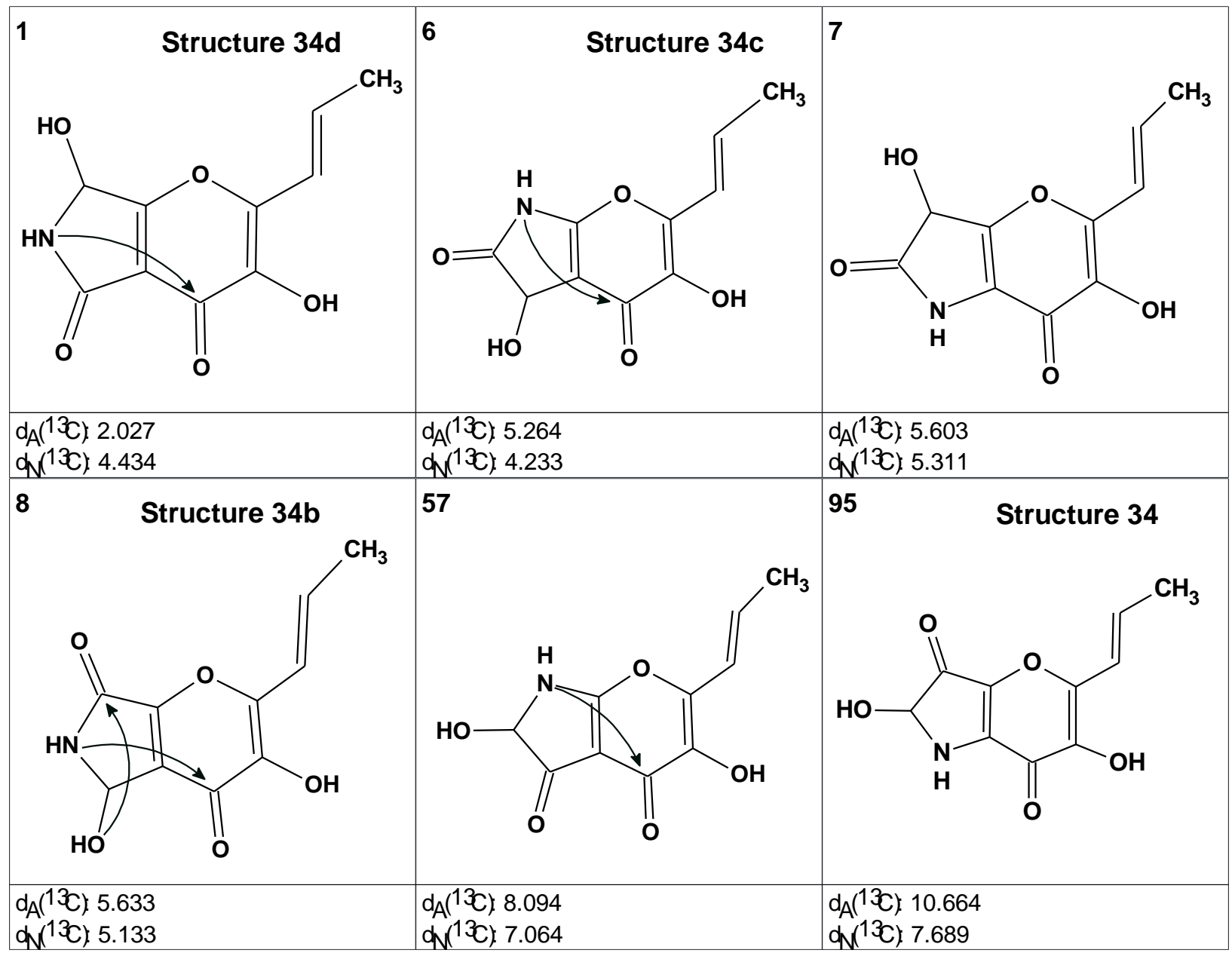


Figure 31. The full set of structures containing all arrangements of $\mathrm{OH}, \mathrm{NH}$ and $\mathrm{C}=\mathrm{O}$ groups on a 5-membered cycle. The numbers in the left upper corner denote the rank of the corresponding structure and the arrows show nonstandard HMBC correlations. The numbers in the top left of each box correspond to the rank ordered structures.

Figure 31 convincingly demonstrates the priority of the correct structure, $\mathbf{3 4 d}$, while the original structure, 34 , was placed in $95^{\text {th }}$ position by the program. Note that the structure ranked as \#7 was the best one in the file obtained by strict structure generation (see Figure 30 ), because only this structure and structure $\mathbf{3 4}$ meet the authors ${ }^{76}$ restrictive suggestion (axiom) regarding the absence of non-standard correlations in the 2D NMR data. Structure 34b could be considered only using the suggestion that it contains two NSCs. For clarity the differences between the original and revised structures are shown in Figure 32

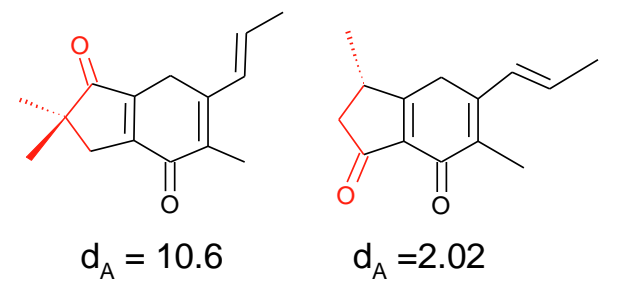

Figure 32. The original and revised structures of pyranonigrin $A$

The example shows that even small molecules with a deficit of hydrogen atoms can become a structure elucidation challenge using traditional approaches. The application of the StrucEluc program would allow Hiort $e t a l^{76}$ to automatically generate all conceivable candidate structures and select the correct molecule in a much reduced time. If only ${ }^{13} \mathrm{C}$ chemical shift prediction was performed for the original structure then it would immediately show that the structure is incorrect since $d_{A}=10.66 \mathrm{ppm}$. New hypotheses would need to be examined.

\subsection{Structure selection on the basis of spectrum prediction}

Johnson $e a^{78}$ reported the unexpected isolation of a novel thiopyrone CTP-431 with molecular formula $\mathrm{C}_{23} \mathrm{H}_{29} \mathrm{NO}_{5} \mathrm{~S}$. On the basis of both mass spectrometry and 2D NMR data (HMQC, HMBC, COSY and NOESY) two possible structures were suggested:
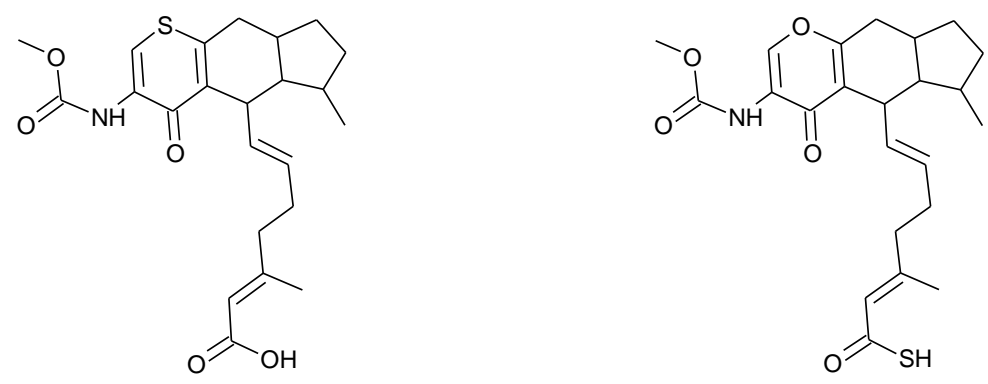
To choose between these two structures, the authors ${ }^{78}$ performed DFT GIAO ${ }^{13} \mathrm{C}$ chemical shift calculations allowing them to select structure $\mathbf{3 5}$ as the most probable. The conclusion was supported by the results of X-ray crystallography.

When StrucEluc was applied the program delivered the following solution from the HMBC data: $\mathrm{k}=408 \rightarrow 273 \rightarrow 273, t_{g}=0.6 \mathrm{~s}$. The top four structures in the ranked output file are presented in Figure 33.

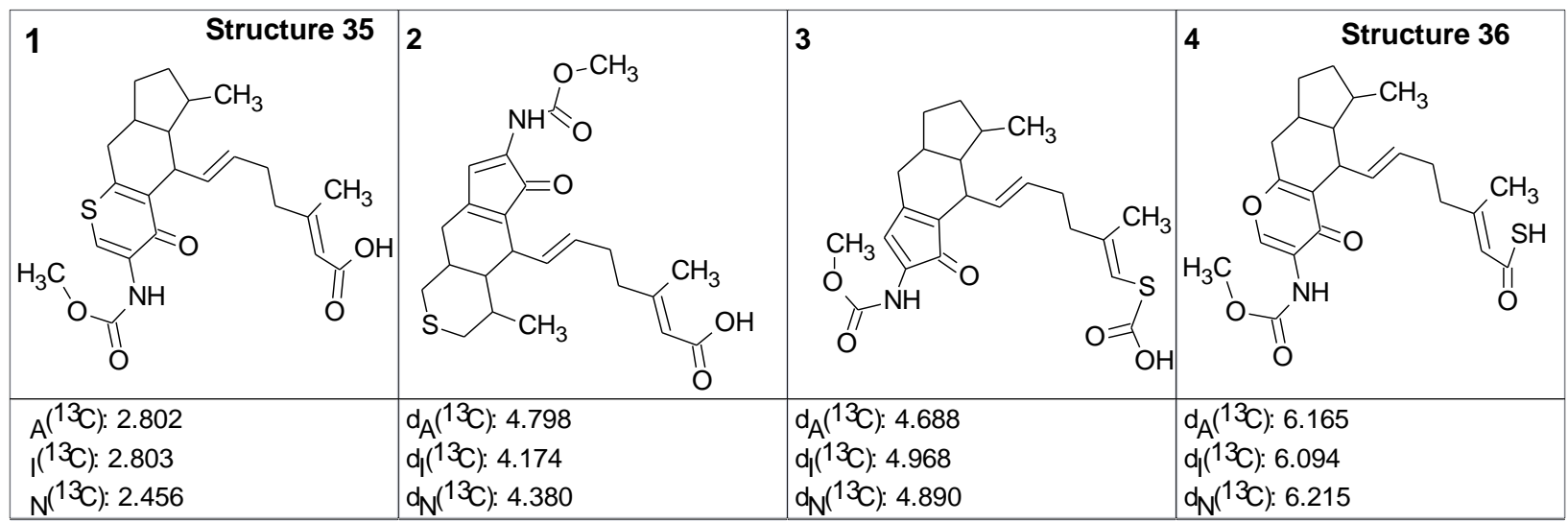

Figure 33. The top ranked structures inferred by the StrucEluc system when the spectral data obtained by Johnson $e t a l^{78}$ were used. The structure of thiopyrone (35) was ranked first by the system. The numbers in the top left of each box correspond to the rank ordered structures.

The figure shows that the correct structure, 35, was reliably distinguished while the alternative structure, 36, was placed only in fourth position in the ranked file. We have previously shown ${ }^{26-28}$ that large deviations (> $6 \mathrm{ppm}$ ) indicate without doubt that structure 36 should be rejected. For clarity the differences between the two competing structures are shown in Figure 34.

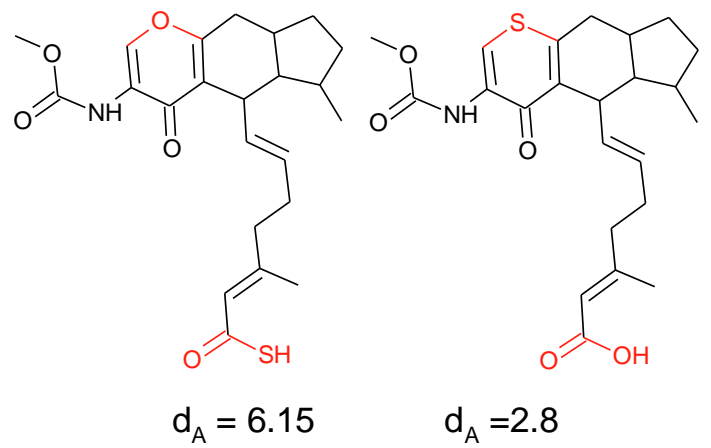

Figure 34. The rejected and real structures of thiopyrone CTP-431

This study indicates that the StrucEluc system can identify the correct structure almost instantly. In connection with this example it should be noted that using only HMBC 
it is not possible to detect the position of the $\mathrm{S}$ atom. However when HMBC is used within StrucEluc in combination with structure generation and ${ }^{13} \mathrm{C}$ NMR spectrum prediction new possibilities arise: the position of the $\mathrm{S}$ atom in the molecule was correctly and quickly detected without time-consuming QM calculations. This demonstrates the strength of the CASE approach.

Takashima et $a l^{79}$ isolated a component from tree bark for which structure $\mathbf{3 7}$ (Brosium allene) was elucidated. The structure assignment was based on high resolution mass spectrometry, ${ }^{1} \mathrm{H},{ }^{13} \mathrm{C}$ and $2 \mathrm{D}$ NMR data. The 2D NMR data were not disclosed.

$\mathrm{Hu}$ et $a l^{80}$ recognized that the ${ }^{13} \mathrm{C}$ NMR signal at $139 \mathrm{ppm}$ was assigned to the central allenic carbon in $\mathbf{3 7}$ even though the central carbon signal of allenes normally appears near $200 \mathrm{ppm}$. This discrepancy served as an impetus for reinvestigation of this compound.

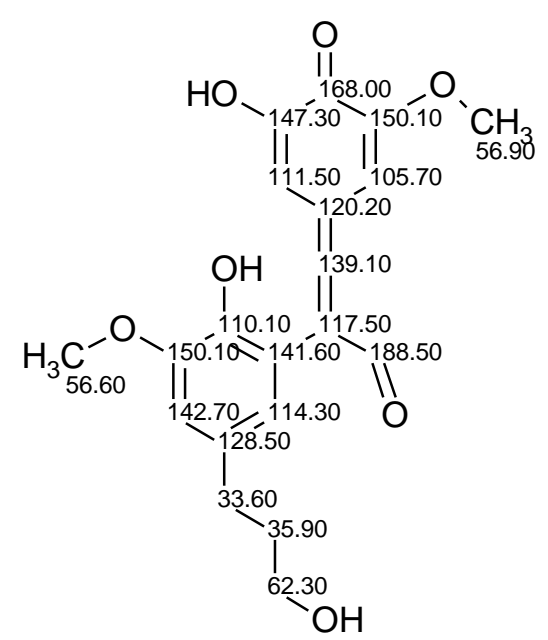

37

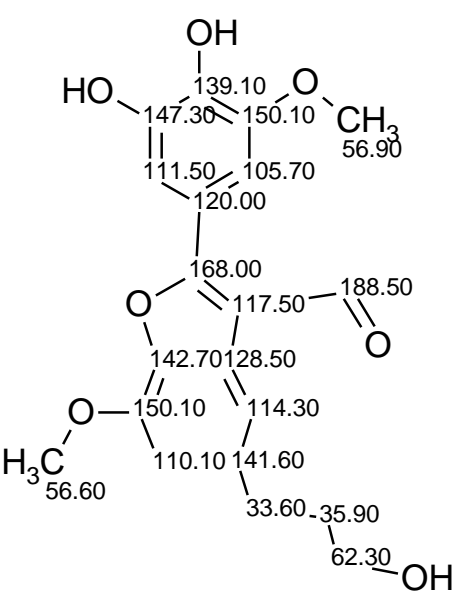

38

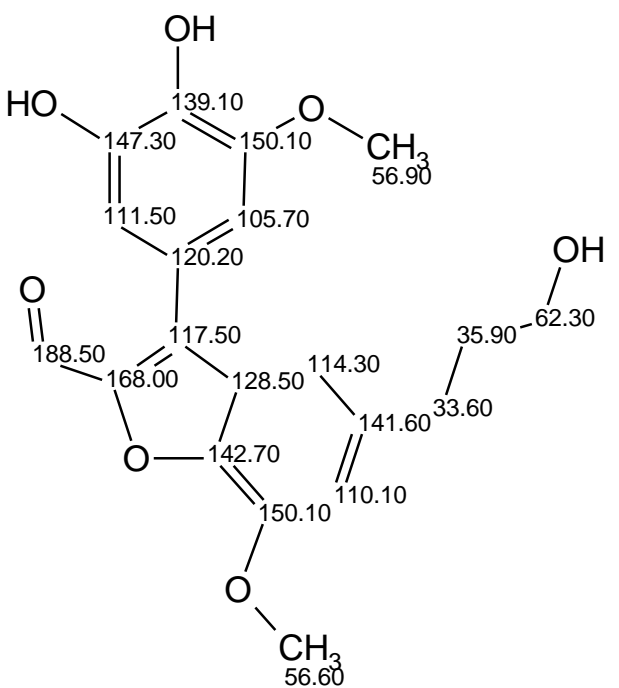

39

The authors ${ }^{80}$ performed quantum-chemical (QM) computational modeling of the ${ }^{13} \mathrm{C}$ chemical shifts expected for 37. Geometry optimizations were performed with B3LYP [6$31 \mathrm{G}(2 \mathrm{~d}, 2 \mathrm{p})]$ and with HF [6-31G $(2 \mathrm{~d}, 2 \mathrm{p})]$. The spectral data were calculated using DFT functionals B3LYP and mPW1PW91, as well as the HF approach. None of the data sets matched well. For the signal assigned as $139 \mathrm{ppm}$ the calculated value was found to consistently be equal to $230 \mathrm{ppm}$. Though QM-based NMR signal prediction is only approximate, a deviation value of $90 \mathrm{ppm}$ is extreme. This observation was considered as evidence that structure $\mathbf{3 7}$ is not correctly assigned. The authors ${ }^{80}$ also doubted that $\mathbf{3 7}$ represents a molecular arrangement isolable under standard conditions.

To verify their suggestion the authors ${ }^{80}$ evaluated the reactivity of structure $\mathbf{3 7}$ and, taking into account the results of the chemical shift predictions, suggested two alternative structures, 38 and 39, as possibilities. QM-based ${ }^{13} \mathrm{C}$ chemical shift prediction for both 
proposed structures led the researchers to conclude that structure $\mathbf{3 8}$ provided the best match between the experimental and calculated values. Finally, the authors showed that structure 38 was identical to a known compound mururin $\mathrm{C}^{81}$.

We also performed ${ }^{13} \mathrm{C}$ chemical shift prediction using our empirical prediction methods $^{43}$ for all three structures. The deviations resulting from the empirical and QM predictions are presented in Figure 35. The figure shows that structure $\mathbf{3 7}$ is rejected by all methods and that structure $\mathbf{3 8}$ is indeed the most probable. It is evident that the StrucEluc system would reject structure 37 if it was generated from 2D NMR data. At the same time Figure 35 demonstrates that the choice of $\mathbf{3 8}$ as the best structure relative to $\mathbf{3 7}$ could be made almost instantly using empirical methods of chemical shift prediction and without the application of time consuming QM calculations.

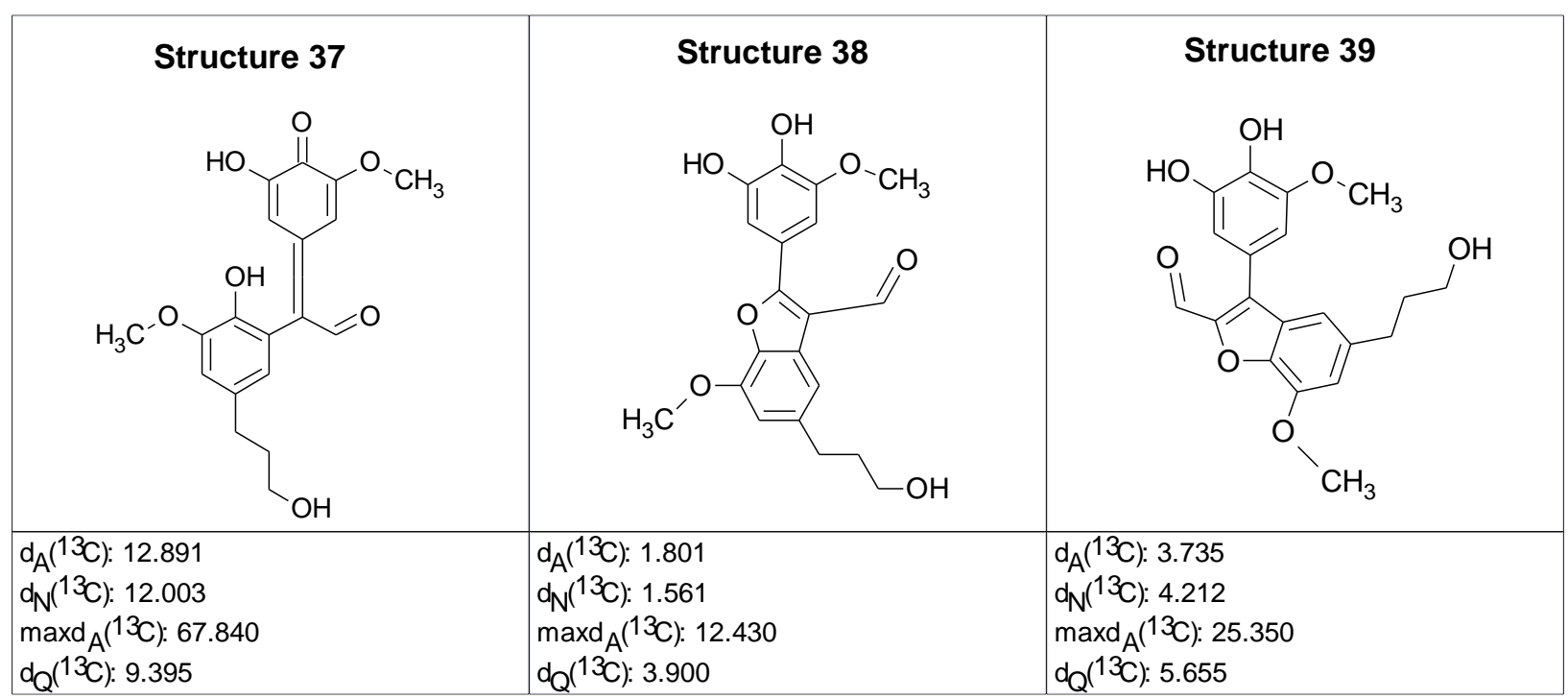

Figure 35. Comparison of discrepancies between experimental and calculated ${ }^{13} \mathrm{C}$ chemical shift for structures 37, 38 and 39. $\mathrm{d}_{\mathrm{Q}}$ is the MAE (mean average deviation) found as a result of QM calculations.

The figure also confirms our previous conclusion ${ }^{82}$ that the accuracy of empirical methods of rapid chemical shift predictions is about two times higher than QM-based predictions. For clarity the differences between the original and revised structures are shown in Figure 36. 


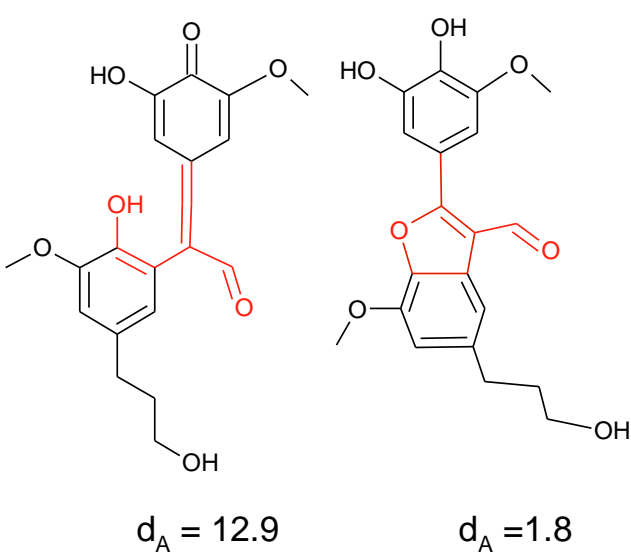

Figure 36. The original and revised structures of Brosium allene.

\section{Conclusions}

In this review we have tried to provide answers to the following important questions: i) are the pitfalls arising during the molecular structure elucidation unavoidable?; and $i$ ) can modern computer-aided methods of molecular structure elucidation be used to minimize the probability of inferring incorrect structures from spectral data?

To investigate these questions we analyzed a large number of examples for which the originally determined structures of novel natural products were revised in later publications. In all cases, when the 2D NMR data were available the expert system Structure Elucidator (reviewed recently ${ }^{33}$ ) was used to determine whether the correct structure could be inferred from the experimental spectra and assumptions or "axioms" suggested by the researcher.

To make the process of structure elucidation more transparent we expounded the main statements of the common methodology describing this process into the form of an axiomatic theory. It has been shown that this theory not only adequately reflects the nature of the problem, but it is also a very important and effective analytical tool which can, and should, be employed routinely in the practice of spectroscopic analysis. This approach appears to be unique for the natural sciences and we failed to find another example of a problem where the initial knowledge could be so clearly and explicitly represented in the form of a set of axioms (hypotheses) and then all logical corollaries, in our case a set of structures, would be automatically inferred then, with subsequent selection, to provide the most probable corollary, in theory the correct chemical structure.

It is also necessary to underline a very important general property of the problem of structure elucidation from spectral data. This problem is related to the class of so-called "inverse problems" $"$. The consequence of this is that a unique and correct solution can be deduced only as a result of using additional information taken from different sources. 
Therefore, the chance of fully replacing human intellect with a computational algorithm is unlikely at best. Moreover, in accordance with the Bohr principle of complementarity ${ }^{84}$, the methodology of computer assisted structure elucidation includes two major elements that complement each other. They are deterministic logic (enhanced with combinatorial analysis) of the computer and the knowledge and intuition of the investigator. The interaction of these elements in the process of solving the problem is what gives rise to the synergistic effect to allow the elucidation of complex molecules. It is therefore necessary to find a rational way of combining connectivities deduced algorithmically from experimental 2D NMR data with additional information such as chemical considerations, hints based on visual spectrum analysis, etc., provided by a scientist in order to obtain a solution to the problem in a reasonable time.

The effectiveness of this relationship between a researcher and a computer accounts for the possibility of the program to produce all consequences, without exception, following from the axiom set provided by the researcher. The many examples presented in this article show that if a researcher's assumptions are incorrect then the solution to a problem is invalid - it does not contain the correct structure.

It has been shown that if the initial NMR data did not contain artifacts and misinterpreted peaks then, in the majority of cases, the software allows the chemist to choose the correct structure. Errors in suggestions made by the researchers or incorrectly interpreted spectral data input into the system leads to output structures whose unlikelihood is easily revealed simply by the application of ${ }^{13} \mathrm{C}$ NMR chemical shift prediction. This allows the researcher to immediately recognize that a particular structural suggestion is not correct or is at least questionable. Figuratively speaking, an expert system can play the role of a "polygraph detector" helping to identify whether a structural hypothesis corresponds to a genuine structure.

As well as ${ }^{13} \mathrm{C}$ chemical shift prediction the dereplication of the structure of any isolated natural product is very useful as a first step towards structure identification. The dereplication process can help to identify the unknown if its structure is already present in a database.

The analysis of the examples in this review allows us to distinguish the following types of errors which are quite commonly made by researchers in the process of forming their initial hypotheses and then in the further deduction of the structure from MS and NMR data: 
- The elemental composition is incorrectly identified to provide the wrong molecular formula.

- Due to insufficient resolution of mass-spectrometer, the $m / z$ value is determined incorrectly. This also leads to an incorrect molecular formula.

- The observation of a spectral feature characteristic for a fragment is erroneously interpreted as evidence of the presence of a particular fragment in a molecule. It should kept in mind that if the implication $\mathrm{A}_{i} \rightarrow \mathrm{X}_{j}$ is true, then the inverse implication $\mathrm{X}_{j} \rightarrow \mathrm{A}_{i}$ can be true or not true.

- Some two-dimensional NMR peaks resulting from a solvent artifact can be erroneously interpreted as part of the 2D NMR spectrum of the unknown compound. As a result the correct structure cannot be inferred. Recording spectra in at least two different solvents can be helpful to detect such issues.

- Some important 2D NMR signals can be missed in the peak picking process and this can certainly prevent generation of the correct structure in certain cases.

- Suggested structures are not checked using the most significant characteristic spectral features in either IR or Raman spectra. For instance, the absence of any absorption in the IR area $3200-3700 \mathrm{~cm}^{-1}$ will reject any hypothetical structure containing an alcohol group.

- The absence of peaks corresponding to expected correlations in an experimental 2D NMR spectrum may be ignored. The spectroscopist is an integral part of the symbiotic partnership between a human and a software program. The highest ranked structures, not the thousands of generated possibilities, should be carefully analyzed in terms of their concordance with the experimental spectra. If the expert using their knowledge and experience determines that one or more expected 2D NMR correlations was not observed then this fact should be a warning as to the plausibility of a structure.

- All 2D NMR correlations are assumed to have only standard lengths. As a result a correct structure whose HMBC or COSY spectra contain nonstandard correlations will be lost.

- The number of nonstandard correlations allowed in 2D NMR data may be incorrectly estimated by the researcher and as a result the correct structure is missed.

- ${ }^{13} \mathrm{C}$ chemical shift prediction might not be performed for the suggested structure. Almost all of the original structures that were identified to be incorrect in this article would have been either rejected or declared suspicious if ${ }^{13} \mathrm{C}$ NMR spectrum 
calculations were performed. There are of course various NMR prediction algorithms and based on our experience and expertise we recommend HOSE-code or neural net algorithms over rules-based approaches.

- When several fragments are deduced from the 2D NMR data by a researcher then the human expert frequently is unable to take into account all possible ways of combining fragments to complete assembly of the structure using, as a rule, HMBC correlations. Many thousands of structures should be checked and as a result the wrong structure may be selected.

When an expert system is employed for the purpose of structure elucidation the overwhelming majority of subjective errors made by the human expert can be either avoided or detected during the process of solving the problem or as a result of validating the most probable structure by NMR spectrum prediction. Some methodological guidelines given below can be helpful.

In general, the process of structure elucidation is $k$ nown ${ }^{27}$ to be reduced to the superposition of constraints on a finite number of isomers that correspond to the molecular formula of an unknown. The number of isomers can be very large even for relatively small molecules ${ }^{27}$. For instance, structure generation using the modest molecular formula $\mathrm{C}_{11} \mathrm{H}_{12} \mathrm{~N}_{4}$ produced $2,258,672,147,012$ isomers ${ }^{85}$. Researchers try to introduce as many as possible constraints to provide a manageable number of suggested structures. As was shown above the issue is that some constraints introduced by user assumptions can be erroneous. The application of an expert system can minimize the number of user assumptions as a result of the high speeds of both structure generation and spectrum prediction: a great number of isomers can be generated in a reasonable time and then fast spectrum prediction allows the program to quickly select the most probable structure. We advise great care when postulating the presence of some fragments and setting atom properties. At the same time the fast NMR prediction algorithms discussed in this review give the user an opportunity to solve the problem repeatedly trying different constraints (spectral and structural hypotheses). Such a solution (structural set) containing a structure characterized by the minimum deviations is considered as at the most preferable one. An expert system also allows the researcher to utilize two or three possible molecular formulae if the elemental composition of the unknown is not clear or the resolving power of the MS instrument is insufficient.

The most challenging part of the structure elucidation process using 2D NMR data is establishing the presence of NSCs, as well as their number and length. To overcome the 
serious difficulties associated with NSCs, the Fuzzy Structure Generation (FSG) algorithm $^{32,34}$ was implemented into StrucEluc. This algorithm is capable of solving a problem under the conditions that neither the number of the NSCs nor their lengths are known. Due to the nature of the sophisticated FSG algorithm not all possible combinations of connectivities are tried, only a small number of them and this dramatically reduces the generation time. The following recommendation is given: if the $d_{A}(1)>3 \mathrm{ppm}$ was found for the highest ranked structure then it is likely incorrect and must be examined further. FSG should initially be performed with $m=1, a=x$ parameters, and if the new $d_{A}(1)$ value reduces in value then there is likely at least one NSC. The typical value of $d_{A}$ acceptable for the correct structure is $1.0-2.5 \mathrm{ppm}$.

In those rare cases when an unknown molecule is classed as "exotic" then the correct structure may be characterized by deviations which are close to or exceeding a threshold of 3 ppm. The reason is that empirical methods are known to exhibit at least one principal drawback: if the database created for the purpose of HOSE prediction, or the training set for the neural net algorithm do not contain specific atoms representing the atom environments in the molecule under investigation, then the empirical methods can fail to predict the chemical shift of such atoms with sufficient accuracy.

Examples of such "exotic" structures are corianlactone (40), hexacyclinol (41), and daphnipaxinin (42), for which $d_{A}$ values were $2.93,3.65$ and 6.34 ppm correspondingly.

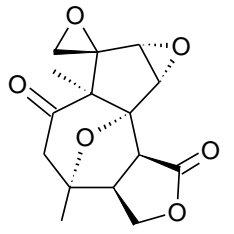

40

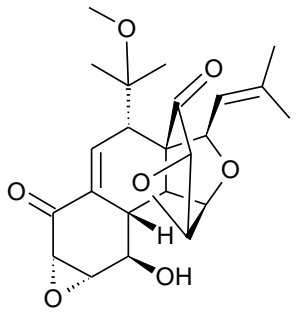

41

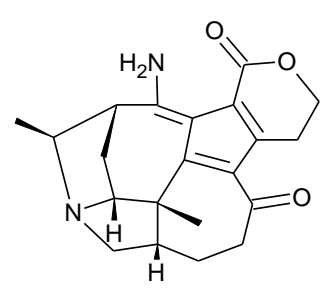

42

We have shown ${ }^{82,86}$ that in spite of the unusual character of these structures and the large values of the deviations, the application of StrucEluc allows the program to correctly select these challenging structures from many candidates while using the structure ranking methodology described above. The intriguing story about the structure elucidation of hexacyclinol was described in a series of publications ${ }^{86-90}$.

${ }^{13} \mathrm{C}$ chemical shift calculation should be considered as the most severe filter to reject all invalid structures and to select the most probable one. However, the average deviations between experimental and predicted spectra that serve as effective criteria for structure assessment are calculable only if chemical shift assignment is completed. The series of examples considered in this review confirm the usefulness of creating linear regression 
plots of plotting calculated ${ }^{13} \mathrm{C}$ chemical shifts against experimental shifts. These graphs allow visual inspection of the point scattering along the full chemical shift scale, while the regression equation and accompanying statistical parameters give numerical criteria for comparing the different suggested structures. A regression plot can also help to detect a small incorrect feature within a molecule when the remaining structure is very close to the correct one (see the halipeptins case).

We have also shown ${ }^{24}$ that if shift assignment is not available, which can happen when CASE methods are not used, then a visual comparison of the graph-bars depicted for the experimental and calculated spectra for a series of suggested structures frequently allows the researcher to identify which structure is the most probable: structures characterized by large outliers should be treated as suspicious.

It would be very attractive to determine some quantitative criteria to allow preliminary estimation of the complexity of a problem. We have failed to find such criteria so far because there are a great number of factors influencing the complexity of the problem and, unfortunately, all of them become known only after a structure is elucidated. Nevertheless, the following properties of the initial data have been identified as factors making solving a problem more difficult:

- a deficit of hydrogen atoms in the molecular formula, and therefore a large value of DBE;

- when the number of experimentally available 2D NMR correlations is markedly less than the number of theoretical correlations for a given structure (discovered $a$ posteriori);

- when there is severe signal overlap in the ${ }^{1} \mathrm{H}$ 2D NMR spectra;

- when the 2D NMR data contain nonstandard correlations;

- when the unknown is very large and contains many heteroatoms.

As mentioned earlier the size of the molecule is not a crucial factor: sufficient 2D NMR correlations allow the system to routinely identify large and complex molecules ${ }^{26,28}$. At the same time even molecules of modest size ( $<15$ skeletal atoms) become difficult to identify when there is a high degree of unsaturation. The histogram of molecular weights of the molecules discussed in this article is presented in Figure 37. The histogram shows that the majority of structures initially elucidated incorrectly are of modest sizes with molecular masses between 200 and 400 Da. 


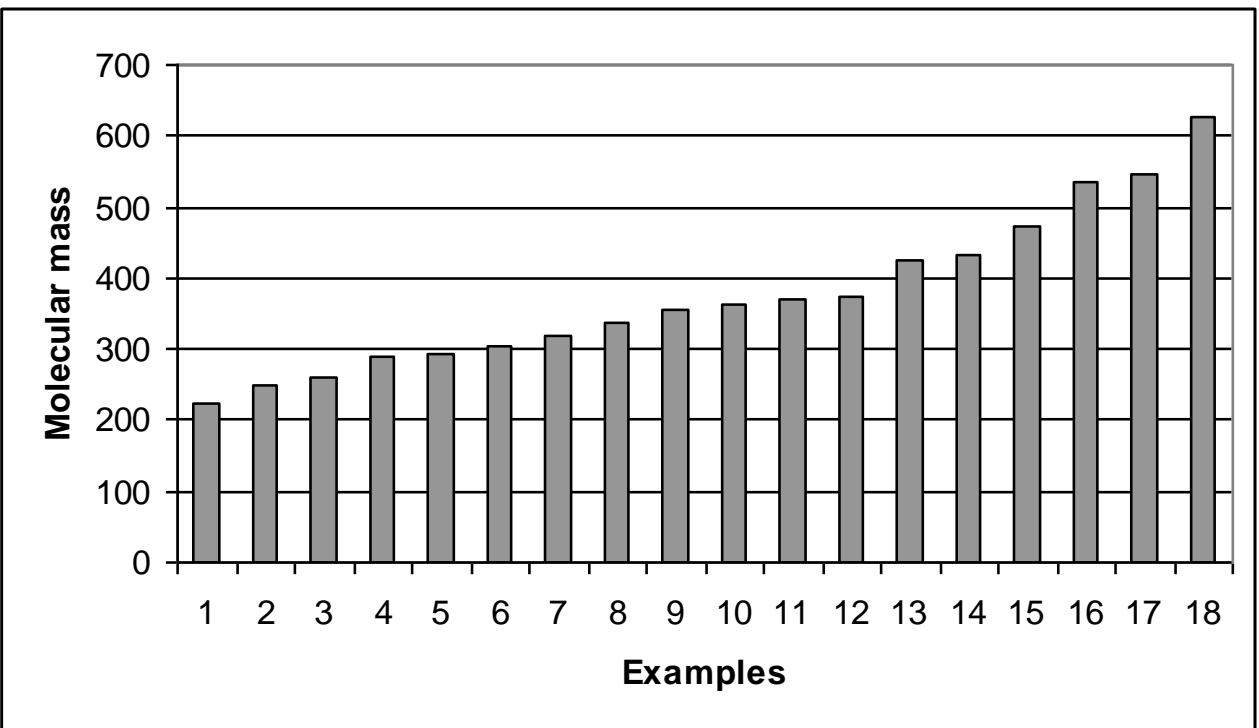

Figure 37. Histogram of molecular weights of examples discussed in this article.

We conclude that the application of expert systems such as Structure Elucidator could dramatically accelerate the structure elucidation of novel natural products, improve the reliability of identification and reduce the number of publications containing erroneous structures. The examples considered in this article clearly demonstrate that an expert system, previously referred to as an artificial intelligence system, is no more than a powerful amplifier of the human intellect. We may expect that as expert system algorithms improve, and computers become faster, then more complex problems will be solvable (as the "gain factor" of the "amplifier" will become higher). We expect that in the near future the further development of expert systems will make such software applications versatile analytical tools that will ultimately become indispensable, not only for structure elucidation but also for the determination of the most probable relative stereochemistry of a newly isolated or synthesized natural product. We also believe that the teaching of CASE methods in universities will help a new generation of chemists to work more efficiently. It will eventually lead to such expert systems becoming routine tools available in the majority of organic and analytical chemistry laboratories. 


\section{References}

1. C. Steinbeck, V. Spitzer, M. Starosta and G. von Poser, J. Nat. Prod., 1997, 60, 627-628.

2. G. N. Belofsky, M. Anguera, P. R. Jensen, W. Fenical and M. Köck, Chem. Eur. J., 2000, 6, $1355-1360$.

3. N. Lysek, E. Rachor and T. Lindel, Z. Naturforsch., 2002, 57C, 1056-1061.

4. D. Mulholland, M. Randrianarivelojosia, C. Lavaud, J.-M. Nuzillard and S. L. Schwikkard, Phytochemistry 2000, 53, 115-118.

5. D. Mulholland, S. L. Schwikkard, P. Sandor and J.-M. Nuzillard, Phytochemistry, 2000, 53, 465-468.

6. J.-P. Bouillon, B. Tinant, J.-M. Nuzillard and C. Portella, Synthesis., 2004, 711-721.

7. G. E. Martin, B. D. Hadden, C. E. Russell, D. J. Kaluzny, J. E. Guido, W. K. Duholke, B. A. Stiemsma, T. J. Thamann, R. C. Crouch, K. A. Blinov, M. E. Elyashberg, E. R. Martirosian, S. G. Molodtsov, A. J. Williams and P. L. J. Schiff, J. Het. Chem., 2002, 39, 1241-1250.

8. K. A. Blinov, M. E. Elyashberg, E. R. Martirosian, S. G. Molodtsov, A. J. Williams, M. M. H. Sharaf, P. L. J. Schiff, R. C. Crouch, G. E. Martin, C. E. Hadden, J. E. Guido and K. A. Mills, Magn. Reson. Chem., 2003, 41, 577-584.

9. G. J. Sharman, Jones I.C., M. J. Parnell, M. Willis, D. V. Carlson, A. Williams, M. E. Elyashberg, K. A. Blinov and S. G. Molodtsov, Magn. Reson. Chem., 2004, 42, 567-572. 
10. M. Jaspars, Nat. Prod. Rep., 1999, 16, 241-248.

11. C. Steinbeck, Nat. Prod. Rep. , 2004, 21, 512-518.

12. M. E. Elyashberg, A. J. Williams and G. E. Martin, Prog. NMR Spectr., 2008, 53, 1-104.

13. Y. Han and C. Steinbeck, J. Chem. Inf. Comput. Sci., 2004, 44, 489-498.

14. T. Lindel, J. Junker and M. Kock, J. Mol. Model. , 1997, 3, 364-368.

15. J.-M. Nuzillard and G. Massiot, Tetrahedron, 1991, 47, 3655-3664.

16. C. Peng, S. Yuan, C. Zheng and Y. Hui, J. Chem. Inf. Comput. Sci., 1994, 34, 805-813.

17. K. P. Schulz, A. Korytko and M. E. Munk, J. Chem. Inf. Comput. Sci., 2003, 43, 14471456.

18. C. Steinbeck, Angew. Chem. Int. Ed. Engl., 1996, 35, 1984-1986

19. C. Steinbeck, J. Chem. Inf. Comput. Sci., 2001, 41, 1500-1507.

20. K. C. Nicolaou and S. A. Snyder, Angew. Chem. Int. Ed. , 2005, 44, 1012-1044.

21. M. E. Maier, Nat. Prod. Rep., 2009, 26, 1105-1124.

22. L. A. Gribov, M. E. Elyashberg and L. A. Moscovkina, J. Mol. Struct. , 1971, 9, 357-371.

23. M. E. Elyashberg, L. A. Gribov and V. V. Serov, Molecular spectral analysis and computers, Nauka, Moscow, 1980.

24. M. E. Elyashberg, K. A. Blinov and A. W. Williams, Magn. Reson. Chem., 2009, 47, 371-389.

25. M. Elyashberg, K. Blinov, A. Williams, S. Molodtsov and E. Martirosian, J. Nat. Prod. , 2002, 65, 693-703.

26. M. E. Elyashberg, K. A. Blinov, S. G. Molodtsov, A. J. Williams and G. E. Martin, J. Chem. Inf. Comput. Sci. , 2004, 44, 771-792.

27. M. E. Elyashberg, K. A. Blinov, A. J. Williams, S. G. Molodtsov and G. E. Martin, J. Chem. Inf. Model. , 2006, 46, 1643-1656.

28. K. A. Blinov, D. Carlson, M. E. Elyashberg, G. E. Martin, E. R. Martirosian, S. G. Molodtsov and A. J. Williams, Magn. Reson. Chem., 2003, 41, 359-372.

29. ACD Structure Elucidator V.12.0. Advanced Chemistry Develpment Inc., (2009).

30. H. Masui and H. Hong, J. Chem. Inf. Model., 2006, 46, 775-787.

31. M. E. Elyashberg, in The Encyclopedia of Computational Chemistry, ed. P. v. R. A. Schleyer, N. L., Clark, T.; Gasteiger, J.; Kollman, P. A.; Schaefer III, H. F.; Schreiner, P. R., John Wiley \& Sons, Chichester, 1998, pp. 1307-1312.

32. S. G. Molodtsov, M. E. Elyashberg, K. A. Blinov, A. J. Williams, G. E. Martin and B. Lefebvre, J. Chem. Inf. Comput. Sci., 2004, 44, 1737-1175.

33. M. E. Elyashberg, K. A. Blinov, S. G. Molodtsov, Y. D. Smurnyy, A. Williams and T. S. Churanova, J. Cheminformatics., 2009, http://www.jcheminf.com/content/1/1/3. 
34. M. E. Elyashberg, K. A. Blinov, A. J. Williams, S. G. Molodtsov and G. E. Martin, J. Chem. Inf. Model. , 2007, 47, 1053-1066.

35. K. A. Blinov, Y. D. Smurnyy, T. S. Churanova, M. E. Elyashberg and A. J. Williams, Chemometr. Intell. Lab. Syst., 2009, 97, 91-97.

36. K. A. Blinov, Y. D. Smurnyy, M. E. Elyashberg, T. S. Churanova, M. Kvasha, C. Steinbeck, B. E. Lefebvre and A. J. Williams, J. Chem. Inf. Model., 2008.

37. Y. D. Smurnyy, K. A. Blinov, T. S. Churanova, M. E. Elyashberg and A. J. Williams, J. Chem. Inf. Model.

, 2008, 48, 128-134.

38. W. Bremser, Anal.Chim. Act. Comp. Techn. Optimiz. , 1978, 2, 355-365.

39. M. Elyashberg, K. Blinov and A. Williams, Magn. Reson. Chem., 2009, 47, 333-341.

40. Y. D. Smurnyy, M. E. Elyashberg, K. A. Blinov, B. Lefebvre, G. E. Martin and A. J. Williams, Tetrahedron, 2005, 61/42 9980-9989.

41. A. Randazzo, G. Bifulco, C. Giannini, M. Bucci, C. Debitus, G. Cirino and L. GomezPaloma, J. Am. Chem. Soc., 2001, 123, 10870-10876.

42. G. Socrates, Infrared and Raman Characteristic Group Frequencies: Tables and Charts. , Wiley, Chichester, 2004.

43. Advanced Chemistry Development. ACD/NMR Predictors. Prediction suite includes $1 H$, 13C, 15N, 19F, 31P NMR prediction, http://www.acdlabs.com.

44. C. D. Monica, A. Randazzo, G. Bifulco, P. Cimino, M. Aquino, I. Izzo, F. De Riccardisc and L. Gomez-Paloma, Tetrahedron Letters 2002, 43.

45. M. E. Elyashberg, Y. Z. Karasev and R. Martirosian, Analyt. Chim. Acta 1999, 388, $353-$ 363.

46. E. Sakuno, K. Yabe, T. Hamasaki and H. Nakajima, J. Nat. Prod. , 2000, 63, 1677-1678.

47. P. Wipf and A. D. Kerekes, J. Nat. Prod. , 2003, 66, 716-718.

48. O. M. Cóbar, A. D. Rodriguez, O. L. Padilla and J. A. Sanchez, J. Org. Chem., 1997, 62, 7183-7188.

49. Y.-P. Shi, A. D. Rodriguez and O. L. Padilla, J. Nat. Prod., 2001, 64, 1439-1443.

50. P. Ralifo and P. Crews, J. Org. Chem. , 2004, 69, 9025-9029.

51. N. Aberle, S. P. B. Ovenden, G. Lessene, K. G. Watson and B. J. Smith, Tetrahedron Lett. , 2007, 48, 2199-2203.

52. K. N. White, T. Amagata, A. G. Oliver, K. Tenney, P. J. Wenzel and P. Crews, J. Org. Chem. , 2008, 73, 8719-8722.

53. A. Buske, S. Busemann, J. Mühlbacher, J. Schmidt, A. Porzel, G. Bringmann and G. Adam, Tetrahedron 1999, 55, 1079-1086. 
54. G. Bringmann, J. Schlauer, H. Rischer, M. Wohlfarth, J. Mühlbacher, A. Buske, A. Porzel, J. Schmidt and G. Adam, Tetrahedron 2000, 56, 3691-3695.

55. P.-W. Hsieh, F.-R. Chang, K.-H. Lee, T.-L. Hwang, S.-M. Chang and Y.-C. Wu, J. Nat. Prod. , 2004, 67, 1175-1177.

56. I. Wetzel, L. Allmendinger and F. Bracher, J. Nat. Prod., 2009, 72, 1908-1910.

57. $\quad$ P.-L. Wu, Y.-L. Hsu and C.-W. Jao, Nat. Prod., 2006, 69, 1467-1470.

58. J. J. Mason, J. Bergman and T. Janosik, J. Nat. Prod. , 2008, 71, 1447-1450.

59. P. Sharma and M. J. Alam, Chem. Soc., Perkin Trans., 1988, 1, 2537.

60. $\quad$ L. A. Paquette, O. M. Moradei, P. Bernardelli and T. Lange, Org. Lett., 2000, 2, 18751878.

61. D. Friedrich, R. W. Doskotch and L. A. Paquette, Org. Lett. , 2000, 2, 1879-1882.

62. D. Friedrich and L. A. Paquette, J. Nat. Prod. , 2002, 65, 126-130.

63. Y. Sakano, M. Shibuya, Y. Yamaguchi, R. Masuma, H. Tomada, S. Omura and Y.

Ebizuka, J. Antibiot. , 2004, 57, 564-568.

64. B. B. Snider and X. Gao, Org. Lett., 2005, 7, 4419-4422.

65. I. H. Hardt, P. R. Jensen and W. Fenical, Tetrahedron Letters 2000, 41, 2073-2076.

66. J. A. Kalaitzis, Y. Hamano, G. Nilsen and B. S. Moore, Org. Lett., 2003, 5, 4449-4452

67. R. Suemitsu, K. Ohnishi, M. Horiuchi, A. Kitagichi and Odamura, Phytochemistry 1992, 31, 2325-2326.

68. M. Horiuchi, T. Maoka, N. Iwase and K. Ohnishi, J. Nat. Prod. , 2002, 65, 1204-1205.

69. T. Komoda, Y. Sugiyama, N. Abe, M. Imachi, H. Hirota and A. Hirota, Tetrahedron Letters 2003, 44, 1659-1661.

70. I. Otani, T. Kusumi, Y. Kashman and H. J. Kakisawa, Am. Chem. Soc. , 1991, 113, 40924096.

71. T. Komoda, Y. Sugiyama, N. Abe, M. Imachi, H. Hirota, H. Koshinoe and A. Hirota, Tetrahedron Letters 2003, 44, 7417-7419.

72. J. Cáceres, M. E. Rivera and A. D. Rodríguez, Tetrahedron, 1990, 46, 341.

73. A. D. Rodríguez, A. L. Acosta and H. Dhasmana, J. Nat. Prod., 1993, 56, 1843-1849.

74. P. Krishnaiah, V. L. N. Reddy, G. Venkataramana, K. Ravinder, M. Srinivasulu, T. V. Raju, K. Ravikumar, D. Chandrasekar, S. Ramakrishna and Y. Venkateswarlu, J. Nat. Prod., 2004, 67, 1168-1171.

75. M. E. Elyashberg, K. A. Blinov, S. G. Molodtsov, T. S. Churanova and A. W. Williams, ChemSpider J. Chem, 2009. 
76. J. Hiort, K. Maksimenka, M. Reichert, S. Perović-Ottstadt, W. H. Lin, V. Wray, K. Steube, K. Schaumann, H. Weber, P. Proksch, R. Ebel, W. E. G. Müller and G. Bringmann, J. Nat. Prod. , 2004, 67, 1532-1543.

77. G. Schlingmann, T. Taniguchi, H. He, R. Bigelis, H. Y. Yang, F. E. Koehn, G. T. Carter and N. Berova, J. Nat. Prod. , 2007, 70, 1180-1187.

78. T. A. Johnson, T. Amagata, A. G. Oliver, K. Tenney, F. A. Valeriote and P. Crews, J. Org. Chem., 2008, 73, 7255-7259 7255.

79. J. Takashima, S. Asano and A. Ohsaki, Tennen Yuki Kagobutsu Toronkai Koen Yoshishu, 2000, 42, 487.

80. G. Hu, K. Liu and L. J. Williams, Org. Lett., 2008, 10, 5493-5496.

81. J. Takashima, S. Asano and A. Ohsaki, Planta Med. , 2002, 68, 621.

82. M. E. Elyashberg, K. A. Blinov, Y. D. Smurnyy, T. S. Churanova and A. J. Williams, Magn. Reson. Chem., 2010, 48, 219-229.

83. L. A. Gribov, M. E. Elyashberg and V. V. Serov, J. Mol. Struct., 1978, 50, 371-387.

84. N. Bohr, Atomic Physics and Human Knowledge, Wiley, New York, 1958.

85. K. A. Blinov, M. E. Elyashberg and A. W. Williams, Unpublished results

86. A. J. Williams, M. E. Elyashberg, K. A. Blinov, D. C. Lankin, G. E. Martin, W. F. Reynolds, J. A. Porco, C. A. Singleton and S. Su, J. Nat. Prod., 2008, 71, 581-588.

87. G. Saielli and A. Bagno, Org. Lett., 2009, 11, 1409-1412.

88. J. A. J. Porco, S. Su, X. Lei, S. Bardhan and S. D. Rychnovsky, Angew. Chem. Int. Ed., 2006, 45, 1-4.

89. S. D. Rychnovsky, Org. Lett., 2006, 8, 2895-2898.

90. J. J. La Clair, Angew. Chem. Int. Ed., 2006, 45, 2769-2773. 